\title{
Discrete Giant Polymeric Chains based on Nanosized Monomers
}

\author{
Zhongguo Liu, ${ }^{1 \dagger}$ Ze Yang, ${ }^{1 \dagger}$ Xin Chen,,${ }^{1 \dagger}$ Rui Tan, ${ }^{1}$ Gang Li, ${ }^{1}$ Zhanhui Gan, ${ }^{1}$ Yu Shao,${ }^{3}$ Jinlin He, ${ }^{4}$ Zhengbiao Zhang, ${ }^{4}$ \\ Weihua Li, ${ }^{5}$ Wen-Bin Zhang, ${ }^{3}$ and Xue-Hui Dong ${ }^{1,2 *}$
}

${ }^{1}$ South China Advanced Institute of Soft Matter Science and Technology, School of Molecular Science and Engineering, South China University of Technology, Guangzhou 510640, China

${ }^{2}$ State Key Laboratory of Luminescent Materials and Devices, South China University of Technology, Guangzhou 510640, China

${ }^{3}$ Key Laboratory of Polymer Chemistry \& Physics of Ministry of Education, Center for Soft Matter Science and Engineering, College of Chemistry and Molecular Engineering, Peking University, Beijing 100871, China

${ }^{4}$ College of Chemistry, Chemical Engineering and Materials Science, Soochow University, Suzhou, 215123, China

${ }^{5}$ State Key Laboratory of Molecular Engineering of Polymers, Department of Macromolecular Science, Fudan University, Shanghai 200433, China

${ }^{\dagger}$ These authors contribute equally

*Corresponding author: xdong@scut.edu.cn 


\section{Materials}

The following chemicals were used as received: octavinylPOSS (Hybrid Plastics), 3-mercapto-1-propanol (Sigma, 95\%), 1-butanethiol (Sigma, 93\%), 2,2,2-trifluoroethanethiol (Sigma, 95\%), benzyl mercaptan (Sigma, 97\%), 1-thioglycerol (Sigma, 98\%), 2,2-dimethoxy-2-phenylacetophenone (DMPA, 98\%, TCI America), maleimide (Sigma, 95\%), diisopropyl azodicarboxylate (DIAD, 97\%, TCI America), dimethylchlorosilane (Sigma, 98\%), hexamethylcyclotrisiloxane (Sigma, 97\%), dimethylphenylsilane (TCI, 97\%), $\mathrm{Pd} / \mathrm{C}$ (Sigma, $10 \%$ loading), tris(pentafluorophenyl)borane $\left(\mathrm{B}\left(\mathrm{C}_{6} \mathrm{~F}_{5}\right)_{3}, \quad 98 \%\right.$, TCI America), triphenylphosphine $\left(\mathrm{PPh}_{3}, 97 \%\right.$, TCI America), tetrabromomethane (CBr4, 98\%, TCI America), potassium thioacetate (KSAc, 98\%, TCI America), furan (Sigma, 98\%), acetyl chloride (98\%, TCI America), anhydrous sodium sulfate $\left(\mathrm{Na}_{2} \mathrm{SO}_{4}\right.$, Sigma, 93\%), tetrahydrofuran (THF), triethylamine (TEA), anhydrous pyridine, dioxane, $1 \mathrm{M}$ phosphate buffer $(\mathrm{pH}=7)$, toluene, petroleum ether (PE), ethyl acetate (EA), methanol $(\mathrm{MeOH})$, chloroform $\left(\mathrm{CHCl}_{3}\right)$, dichloromethane $\left(\mathrm{CH}_{2} \mathrm{Cl}_{2}\right)$. Azodiisobutyronitrile (AIBN, 98\%, TCI America) was recrystallized from methanol three times before used. Anhydrous solvents, including toluene, dichloromethane, $N, N^{\prime}$-dimethylformamide (DMF), were obtained with an INERT Pure Solv System (Inert Corporation, USA).

\section{Characterizations}

Nuclear Magnetic Resonance (NMR). All ${ }^{1} \mathrm{H}$ NMR spectra were acquired in $\mathrm{CDCl}_{3}$ using a Bruker 400 MHz NMR spectrometer. The spectra were referenced to the residual solvent peak in $\mathrm{CDCl}_{3}$ at $\delta 7.27 \mathrm{ppm}$.

${ }^{29} \mathrm{Si}$ NMR spectra were acquired in $\mathrm{CDCl}_{3}$ using a Bruker $99 \mathrm{MHz} \mathrm{NMR}$ spectrometer and referenced to tetramethylsilane (TMS) at $\delta 0.00 \mathrm{ppm}$.

Size exclusion chromatography (SEC). SEC analyses were measured at $40{ }^{\circ} \mathrm{C}$ on a Tosoh HLC-8320 instrument equipped with three TSKgel columns (SuperH2000, SuperH3000, and SuperH4000 in series), a double flow type RI detector, and a UV-8320 UV detector, using THF as eluent. The flow-rate was $0.6 \mathrm{~mL} / \mathrm{min}$. 
Data acquisition was performed using EcoSEC software, and molecular weights and molecular weight distributions were calibrated with polystyrene standards (Polymer Laboratories).

Matrix-assisted laser desorption/ionization time-of-flight (MALDI-ToF). MALDI-ToF mass spectroscopy (MS) were acquired on an UltrafleXtreme MALDI-ToF mass spectrometer (Bruker Daltonics) equipped with a $1 \mathrm{kHz}$ smart beam-II laser. Trans-2-[3-(4-tert-butyl-phenyl)-2-methyl-2-propenylidene]-malononitrile (DCTB, Sigma-Aldrich, $>98 \%$ ) was used as the matrix and prepared in $\mathrm{CHCl}_{3}$ at a concentration of $20 \mathrm{mg} / \mathrm{mL}$. The cationizing agent sodium trifluoroacetate was prepared in ethanol at a concentration of $10 \mathrm{mg} / \mathrm{mL}$. The matrix and cationizing salt solutions were mixed in a ratio of 10/1 (v/v). All samples were dissolved in $\mathrm{CHCl}_{3}$ at a concentration of $10 \mathrm{mg} / \mathrm{mL}$. After sample preparation and solvent evaporation, the target plate was inserted into the MALDI-ToF mass spectrometer. The attenuation of the laser was adjusted to minimize undesired polymer fragmentation and maximize the sensitivity.

Thermogravimetric Analysis (TGA). Nonisothermal decomposition experiments were carried out on a thermogravimetric analysis instrument (Model Q500, TA Instruments) under nitrogen atmosphere protection. For each run, initial mass of the samples was about $3 \mathrm{mg}$. The samples were scanned from 30 to $500^{\circ} \mathrm{C}$ with a heating rate of $10^{\circ} \mathrm{C} / \mathrm{min}$.

Differential Scanning Calorimetry (DSC). Thermal transitions of all the samples were characterized utilizing a PerkinElmer PYRIS Diamond DSC with an Intracooler 2P apparatus. The samples were sealed in a DSC pan with an initial mass of about 5-8 $\mathrm{mg}$ and scanned from -60 to $80{ }^{\circ} \mathrm{C}$ with a heating/cooling rate of $5{ }^{\circ} \mathrm{C} / \mathrm{min}$ under nitrogen flow. 


\section{Syntheses}

\subsection{POSS Monomers with Diverse Surface Functionalities}

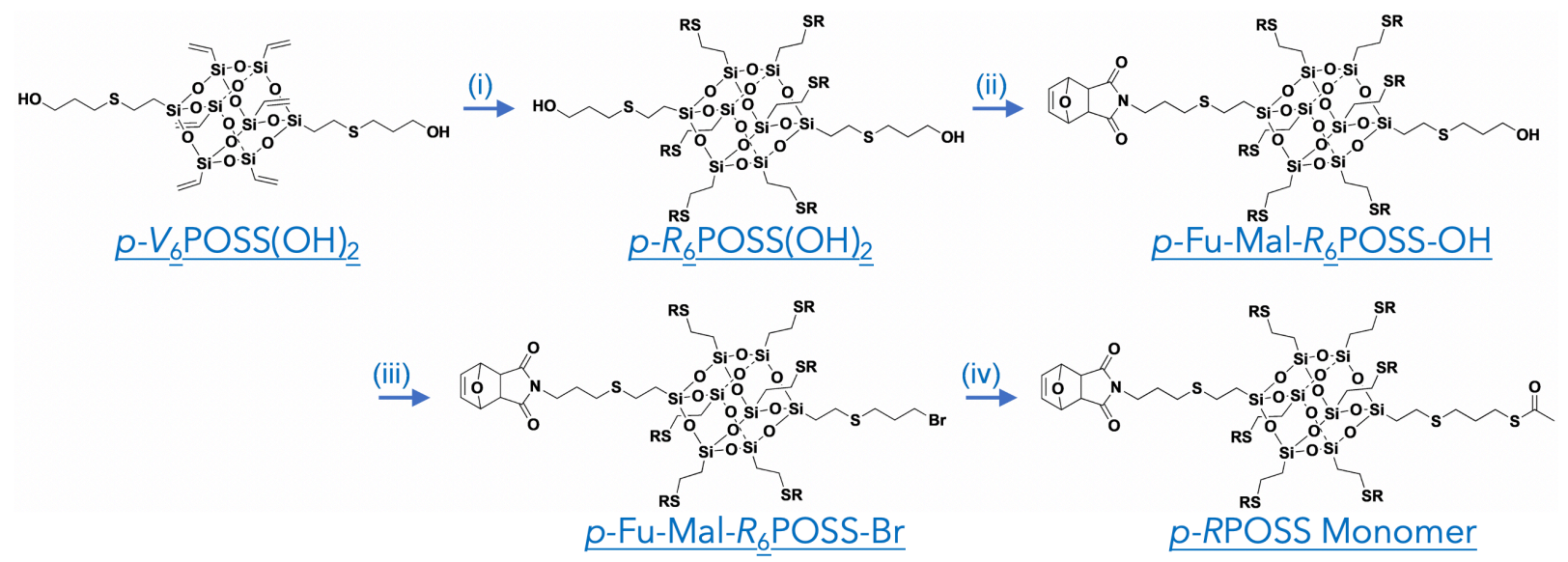

Scheme S1. Synthetic route of the POSS based monomers with diverse functionalities: (i) RSH, DMPA, THF, $25^{\circ} \mathrm{C}$, $365 \mathrm{~nm} \times 30 \mathrm{~min}$; (ii) Furan protected maleimide, DIAD, $\mathrm{PPh}_{3}$, THF, $0{ }^{\circ} \mathrm{C}, 12 \mathrm{~h}$; (iii) $\mathrm{CBr}_{4}, \mathrm{PPh}_{3}, \mathrm{CH}_{2} \mathrm{Cl}_{2}, 0^{\circ} \mathrm{C}, 2$ h; (iv) potassium thioacetate, $\mathrm{KI}, \mathrm{THF} / \mathrm{MeOH}, 75^{\circ} \mathrm{C}, 12 \mathrm{~h}$.

3.1.1 Furan-protected maleimide. To a $250 \mathrm{~mL}$ round-bottom flask were added with maleimide $(9.7 \mathrm{~g}$, $0.1 \mathrm{~mol})$ and furan $(13.6 \mathrm{~g}, 0.2 \mathrm{~mol})$. The mixture was dissolved in $160 \mathrm{~mL}$ of toluene. The flask was sealed and heated at $90{ }^{\circ} \mathrm{C}$ for $12 \mathrm{~h}$. After cooling to room temperature, the product precipitated out as a white solid. The mixture was filtered and the filter cake was washed with $3 \times 150 \mathrm{~mL}$ cold toluene. The product was dried under vacuum at $25^{\circ} \mathrm{C}$ overnight to afford furan-protected maleimide as a white crystal (14.7 g, yield: $\left.89 \%\right)$.

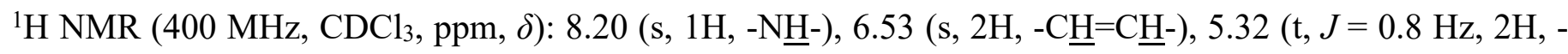

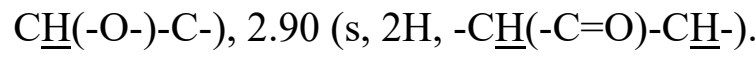

3.1.2 para-, meta-, ortho-V ${ }_{6} \mathbf{P O S S}(\mathrm{OH})_{2}$. OctavinylPOSS (30.0 g, $\left.47.4 \mathrm{mmol}\right)$, 3-mercapto-1-propanol (8.72 g, $94.8 \mathrm{mmol})$, and DMPA (140 mg, $0.55 \mathrm{mmol})$ were dissolved in $350 \mathrm{~mL}$ of THF, and irradiated with UV (365 $\mathrm{nm}$ ) for $30 \mathrm{~min}$. After removing solvent, the regio-isomers were isolated by silica gel chromatography. A gradient mixture of EA/DCM (v/v from 1/20 to 1/4) was used as eluents to afford the products as white 
powders. Each fraction was characterized by ${ }^{1} \mathrm{H}$ and ${ }^{29} \mathrm{Si}$ NMR spectrometry (Figure S1).

p-V6 ${ }_{6} \mathbf{P O S S}(\mathbf{O H})_{2}\left(1.2 \mathrm{~g}\right.$, yield: 3.1\%). ${ }^{1} \mathrm{H}$ NMR (400 MHz, $\left.\mathrm{CDCl}_{3}, \mathrm{ppm}, \delta\right): 6.15-5.87\left(\mathrm{~m}, 18 \mathrm{H},-\underline{\mathrm{H}}=\underline{\mathrm{C}}_{2}\right)$, $3.76\left(\mathrm{t}, J=6.0 \mathrm{~Hz}, 4 \mathrm{H},-\underline{\mathrm{C}}_{2} \mathrm{OH}\right), 2.69-2.65\left(\mathrm{~m}, 8 \mathrm{H},-\mathrm{CH}_{2} \mathrm{C}_{2} \mathrm{SC}_{2} \mathrm{CH}_{2}-\right), 1.85(\mathrm{tt}, J=7.1,6.0 \mathrm{~Hz}, 4 \mathrm{H},-$ $\left.\mathrm{SCH}_{2} \underline{\mathrm{C}}_{2} \mathrm{CH}_{2} \mathrm{OH}\right), 1.07$ (m, 4H, - $\left.\mathrm{SiC}_{2}-\right) .{ }^{13} \mathrm{C} \mathrm{NMR}\left(77 \mathrm{MHz}, \mathrm{CDCl}_{3}, \mathrm{ppm}, \delta\right.$ ): 137.14, 128.65, 61.77, 31.77, 28.44, 25.94, 13.04. ${ }^{29} \mathrm{Si} \mathrm{NMR}\left(99 \mathrm{MHz}, \mathrm{CDCl}_{3}, \mathrm{ppm}, \delta\right):-68.53,-80.35$.

$\boldsymbol{m}-V_{6} \mathbf{P O S S}(\mathbf{O H})_{2}\left(2.8 \mathrm{~g}\right.$, yield: 7.2\%). ${ }^{1} \mathrm{H} \mathrm{NMR}\left(400 \mathrm{MHz}, \mathrm{CDCl}_{3}, \mathrm{ppm}, \delta\right): 6.15-5.86\left(\mathrm{~m}, 18 \mathrm{H},-\underline{\mathrm{H}}=\mathrm{C}_{2}\right)$, $3.76\left(\mathrm{t}, J=6.1 \mathrm{~Hz}, 4 \mathrm{H},-\underline{\mathrm{C}}_{2} \mathrm{OH}\right), 2.69-2.66\left(\mathrm{~m}, 8 \mathrm{H},-\mathrm{CH}_{2} \underline{\mathrm{CH}}_{2} \mathrm{SC}_{2} \mathrm{CH}_{2}-\right), 1.84(\mathrm{tt}, J=7.0,6.0 \mathrm{~Hz}, 4 \mathrm{H},-$

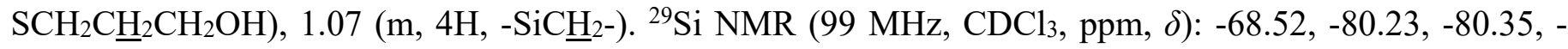
80.48

$\boldsymbol{o}-\mathbf{V}_{6} \mathbf{P O S S}(\mathbf{O H})_{2}\left(3.5 \mathrm{~g}\right.$, yield: 9.1\%). ${ }^{1} \mathrm{H}$ NMR (400 MHz, $\left.\mathrm{CDCl}_{3}, \mathrm{ppm}, \delta\right): 6.14-5.87\left(\mathrm{~m}, 18 \mathrm{H},-\underline{\mathrm{H}}=\mathrm{C}_{2}\right)$, $3.76\left(\mathrm{t}, J=6.0 \mathrm{~Hz}, 4 \mathrm{H},-\underline{\mathrm{H}}_{2} \mathrm{OH}\right), 2.69-2.64\left(\mathrm{~m}, 8 \mathrm{H},-\mathrm{CH}_{2} \mathrm{C}_{2} \mathrm{SC}_{2} \mathrm{CH}_{2}-\right), 1.85(\mathrm{tt}, J=7.0,6.1 \mathrm{~Hz}, 4 \mathrm{H},-$ $\mathrm{SCH}_{2} \underline{\mathrm{CH}}_{2} \mathrm{CH}_{2} \mathrm{OH}$ ), 1.08 (m, 4H, - $\mathrm{SiCH}_{2}-$ ). ${ }^{29} \mathrm{Si} \mathrm{NMR}$ (99 MHz, $\mathrm{CDCl}_{3}, \mathrm{ppm}, \delta$ ): -68.63, -80.22, -80.36.

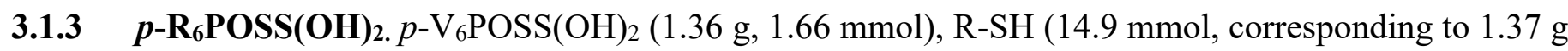
of 1-butanethiol, $1.49 \mathrm{~g}$ of 2,2,2-trifluoroethanol, or $1.85 \mathrm{~g}$ of benzyl mercaptan), and DMPA (41.0 mg, 0.16 mmol) were dissolved in $13 \mathrm{~mL}$ of THF. After UV irradiation (365 nm) for $30 \mathrm{~min}$, solvent was removed, and the residue was purified by silica gel chromatography with PE/EA $(4 / 1, v / v)$ as eluent to afford the products (colorless oil liquids).

p-C6 $\mathbf{P O S S}(\mathbf{O H})_{2}\left(1.68 \mathrm{~g}\right.$, yield: 74.6\%). ${ }^{1} \mathrm{H} \mathrm{NMR}\left(400 \mathrm{MHz}, \mathrm{CDCl}_{3}, \mathrm{ppm}, \delta\right.$ ): 3.76 (q, $J=5.8 \mathrm{~Hz}, 4 \mathrm{H}$, $\left.\mathrm{HOC}_{2} \mathrm{CH}_{2}-\right), 2.50-2.67\left(\mathrm{~m}, 32 \mathrm{H},-\underline{\mathrm{C}}_{2} \mathrm{SC}_{2}-\right), 1.86\left(\mathrm{~h}, J=7.0 \mathrm{~Hz}, 4 \mathrm{H},-\mathrm{SCH}_{2} \mathrm{C}_{2} \mathrm{CH}_{2} \mathrm{OH}\right), 1.56(\mathrm{~m}, 12 \mathrm{H},-$ $\left.\mathrm{SCH}_{2} \underline{\mathrm{C}}_{2} \mathrm{CH}_{2} \mathrm{CH}_{3}\right), 1.43\left(\mathrm{~h}, J=7.3 \mathrm{~Hz}, 12 \mathrm{H},-\mathrm{SCH}_{2} \mathrm{CH}_{2} \underline{\mathrm{C}}_{2} \mathrm{CH}_{3}\right), 1.05\left(\mathrm{~m}, 16 \mathrm{H},-\mathrm{SiC}_{2}-\right), 0.94(\mathrm{~m}, 18 \mathrm{H},-$ $\left.\mathrm{CH}_{2} \mathrm{CH}_{2} \mathrm{CH}_{3}\right) .{ }^{13} \mathrm{C}$ NMR (77 MHz, $\left.\mathrm{CDCl}_{3}, \mathrm{ppm}, \delta\right): 61.58,31.98,31.57,28.46,25.92,22.04,13.72,13.01$, 12.88 
p-F6POSS(OH)2 (1.97 g, yield: 78.4\%). ${ }^{1} \mathrm{H}$ NMR (400 MHz, $\mathrm{CDCl}_{3}, \mathrm{ppm}, \delta$ ): 3.77 (q, $J=5.2 \mathrm{~Hz}, 4 \mathrm{H}$, $\left.\mathrm{HOC}_{2} \mathrm{CH}_{2}-\right), 3.12\left(\mathrm{q}, J=9.9 \mathrm{~Hz}, 12 \mathrm{H},-\mathrm{SC}_{2} \mathrm{CF}_{3}\right), 2.46-2.68\left(\mathrm{~m}, 20 \mathrm{H},-\underline{\mathrm{H}}_{2} \mathrm{SCH}_{2} \mathrm{CF}_{3},-\underline{\mathrm{C}}_{2} \mathrm{SC}_{2} \mathrm{CH}_{2}-\right)$,

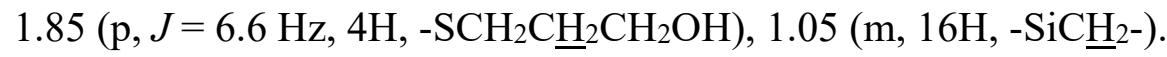

p-P6POSS(OH)2 $\left(2.21 \mathrm{~g}\right.$, yield:85.3\%). ${ }^{1} \mathrm{H}$ NMR (400 MHz, $\left.\mathrm{CDCl}_{3}, \mathrm{ppm}, \delta\right)$ : 7.23-7.35 (m, 30H, $\left.-\mathrm{SCH}_{2} \underline{\mathrm{Ph}}\right)$, 3.74 (q, $\left.J=4.9 \mathrm{~Hz}, 4 \mathrm{H}, \mathrm{HOCH}_{2} \mathrm{CH}_{2}-\right), 3.70\left(\mathrm{~m}, 14 \mathrm{H},-\mathrm{SC}_{2} \mathrm{Ph}\right), 2.41-2.65\left(\mathrm{~m}, 20 \mathrm{H},-\underline{\mathrm{C}}_{2} \mathrm{SCH}_{2} \mathrm{Ph},-\right.$ $\left.\mathrm{C}_{2} \mathrm{SC}_{2} \mathrm{CH}_{2}-\right), 1.84\left(\mathrm{p}, J=6.0 \mathrm{~Hz}, 4 \mathrm{H},-\mathrm{SCH}_{2} \underline{\mathrm{C}}_{2} \mathrm{CH}_{2} \mathrm{OH}\right), 1.01\left(\mathrm{~m}, 16 \mathrm{H},-\mathrm{SiC}_{2}-\right)$.

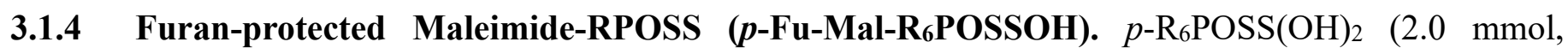
corresponding to $2.72 \mathrm{~g}$ of $\mathrm{C}_{6} \mathrm{POSS}(\mathrm{OH})_{2}, 3.03 \mathrm{~g}$ of $\mathrm{F}_{6} \mathrm{POSS}(\mathrm{OH})_{2}$, or $3.13 \mathrm{~g}$ of $\left.\mathrm{P}_{6} \mathrm{POSS}(\mathrm{OH})_{2}\right)$, furanprotected maleimide $(0.33 \mathrm{~g}, 2.0 \mathrm{mmol})$, and triphenylphosphine $\left(\mathrm{PPh}_{3}, 0.79 \mathrm{~g}, 3.0 \mathrm{mmol}\right)$ were added to a round-bottom flask with anhydrous THF $(25 \mathrm{~mL})$, purged with nitrogen. The reaction mixture was stirred and cooled to $0{ }^{\circ} \mathrm{C}$ in an ice bath and diisopropyl azodicarboxylate (DIAD, $0.61 \mathrm{~g}, 3.0 \mathrm{mmol}$ ) was added dropwise. The solution was allowed to stir for $8 \mathrm{~h}$ at room temperature. The solvent was removed under reduced pressure and the crude product was purified by silica gel column chromatography with PE/EA (3/1, v/v) as eluent to afford Fu-Mal-R6POSSOH.

p-Fu-Mal-C 6 POSSOH (1.76 g, yield: 58.6\%). ${ }^{1} \mathrm{H}$ NMR (400 MHz, $\left.\mathrm{CDCl}_{3}, \mathrm{ppm}, \delta\right): 6.52(\mathrm{~d}, J=0.9 \mathrm{~Hz}, 2 \mathrm{H}$, $-\mathrm{C} \underline{\mathrm{H}}=\mathrm{C} \underline{\mathrm{H}}-), 5.26$ (d, $J=1.0 \mathrm{~Hz}, 2 \mathrm{H},-\mathrm{C} \underline{\mathrm{H}}(-\mathrm{O}-) \mathrm{C}-), 3.75$ (t, $\left.J=6.1 \mathrm{~Hz}, 2 \mathrm{H}, \mathrm{HOC}_{2} \mathrm{CH}_{2}-\right), 3.58(\mathrm{t}, J=7.1 \mathrm{~Hz}$, $\left.2 \mathrm{H},-\mathrm{NC}_{2} \mathrm{CH}_{2}-\right), 2.85(\mathrm{~s}, 2 \mathrm{H},-\mathrm{C} \underline{\mathrm{H}}(-\mathrm{C}=\mathrm{O}) \mathrm{C} \underline{\mathrm{H}}-), 2.52-2.65\left(\mathrm{~m}, 32 \mathrm{H},-\underline{\mathrm{C}}_{2} \mathrm{SC}_{2}-\right), 1.84 \quad(\mathrm{~m}, 4 \mathrm{H},-$ $\left.\mathrm{CH}_{2} \underline{\mathrm{C}}_{2} \mathrm{CH}_{2}-\right), 1.57\left(\mathrm{~m}, 12 \mathrm{H},-\underline{\mathrm{CH}}_{2} \mathrm{CH}_{2} \mathrm{CH}_{3}\right), 1.42$ (dq, $\left.J=14.6,7.3 \mathrm{~Hz}, 12 \mathrm{H},-\mathrm{CH}_{2} \mathrm{C}_{2} \mathrm{CH}_{3}\right), 1.02(\mathrm{~m}, 16 \mathrm{H}$, $\left.-\mathrm{SiC}_{2} \mathrm{CH}_{2}-\right), 0.93\left(\mathrm{~m}, 18 \mathrm{H},-\mathrm{CH}_{2} \mathrm{CH}_{2} \underline{\mathrm{C}}_{3}\right) .{ }^{13} \mathrm{C} \mathrm{NMR}\left(77 \mathrm{MHz}, \mathrm{CDCl}_{3}, \mathrm{ppm}, \delta\right): 176.07,136.38,80.82$, $61.35,47.32,37.83,31.91,31.38,28.05,28.29,27.10,25.77,21.91,13.62,12.86,12.79$.

p-Fu-Mal-F6POSSOH (1.71 g, yield: 51.6\%). ${ }^{1} \mathrm{H}$ NMR (400 MHz, $\left.\mathrm{CDCl}_{3}, \mathrm{ppm}, \delta\right): 6.52(\mathrm{~d}, J=1.0 \mathrm{~Hz}, 2 \mathrm{H}$, $-\mathrm{C} \underline{\mathrm{H}}=\mathrm{C} \underline{\mathrm{H}}-), 5.24$ (d, $J=0.9 \mathrm{~Hz}, 2 \mathrm{H},-\mathrm{C} \underline{\mathrm{H}}(-\mathrm{O}-) \mathrm{C}-), 3.78$ (t, $\left.J=6.0 \mathrm{~Hz}, 2 \mathrm{H}, \mathrm{HOC}_{2} \mathrm{CH}_{2}-\right), 3.59$ (t, $J=5.6 \mathrm{~Hz}$, 


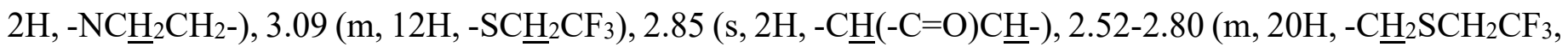
$\left.-\underline{\mathrm{CH}}_{2} \mathrm{SC}_{2} \mathrm{CH}_{2}-\right), 1.85$ (m, 4H, - $\left.\mathrm{CH}_{2} \underline{\mathrm{CH}}_{2} \mathrm{CH}_{2}-\right), 1.05$ (m, 16H, $\left.-\mathrm{SiC}_{2} \mathrm{CH}_{2}-\right)$.

p-Fu-Mal-P6POSSOH (1.88 g, yield: 54.9\%). ${ }^{1} \mathrm{H}$ NMR (400 MHz, $\left.\mathrm{CDCl}_{3}, \mathrm{ppm}, \delta\right):$ 7.22-7.34 (m, 30H, $\left.\mathrm{SCH}_{2} \underline{\mathrm{Ph}}\right), 6.48(\mathrm{~d}, J=1.0 \mathrm{~Hz}, 2 \mathrm{H},-\mathrm{C} \underline{\mathrm{H}}=\mathrm{C} \underline{\mathrm{H}}-), 5.25$ (t, $\left.J=0.8 \mathrm{~Hz}, 2 \mathrm{H},-\mathrm{C} \underline{\mathrm{H}}(-\mathrm{O}-) \mathrm{C}-\right), 3.75-3.70$ (m, 14H, $\left.\mathrm{HOC}_{2} \mathrm{CH}_{2}-,-\mathrm{SC}_{2} \mathrm{Ph}\right), 3.58\left(\mathrm{t}, J=7.0 \mathrm{~Hz}, 2 \mathrm{H},-\mathrm{NC}_{2} \mathrm{CH}_{2}-\right), 2.82(\mathrm{~s}, 2 \mathrm{H},-\mathrm{C} \underline{\mathrm{H}}(-\mathrm{C}=\mathrm{O}) \mathrm{C} \underline{\mathrm{H}}-), 2.40-2.64(\mathrm{~m}$, $\left.20 \mathrm{H},-\mathrm{C} \underline{H} 2 \mathrm{SCH}_{2} \mathrm{Ph}-\underline{\mathrm{C}}_{2} \mathrm{SC}_{2} \mathrm{CH}_{2}-\right), 1.85\left(\mathrm{~m}, 4 \mathrm{H},-\mathrm{CH}_{2} \mathrm{C}_{2} \mathrm{CH}_{2}-\right), 1.01$ (m, 16H, - $\left.\mathrm{SiC}_{2} \mathrm{CH}_{2}-\right)$.

3.1.5 $p$-RPOSS monomer. $p$-Fu-Mal-R ${ }_{6} P O S S O H(1.0 \mathrm{mmol}$, corresponding to $1.51 \mathrm{~g}$ of $p$-Fu-Mal$\mathrm{C}_{6} \mathrm{POSSOH}, 1.66 \mathrm{~g}$ of $p$-Fu-Mal-F 6 POSSOH, or $1.71 \mathrm{~g}$ of $p$-Fu-Mal-P $\mathrm{POSSOH}_{6}$ ) was dissolved in $15 \mathrm{~mL}$ of anhydrous $\mathrm{CH}_{2} \mathrm{Cl}_{2}$ in a $25 \mathrm{~mL}$ round-bottom flask. $\mathrm{CBr}_{4}(0.66 \mathrm{~g}, 2.0 \mathrm{mmol})$ was then added, and the mixture was transferred into an ice bath. When completely dissolved, $\mathrm{PPh}_{3}(0.52 \mathrm{~g}, 2.0 \mathrm{mmol})$ was slowly added and the ice bath was removed. After string for 4 hours, $\mathrm{MeOH}(20 \mathrm{~mL}), \mathrm{KI}(20 \mathrm{mg})$, and potassium thioacetate $(0.23 \mathrm{~g}, 2.0 \mathrm{mmol})$ were added, and the mixture was further stirred at $75^{\circ} \mathrm{C}$ for $12 \mathrm{~h}$ under argon atmosphere. The solvent was then removed under vacuum and the crude product was purified by silica gel column chromatography with PE/EA (5/1, v/v) as eluent to afford monomers.

p-CPOSS monomer (1.32 g, yield: 84.3\%). ${ }^{1} \mathrm{H}$ NMR (400 MHz, $\left.\mathrm{CDCl}_{3}, \mathrm{ppm}, \delta\right): 6.51(\mathrm{~d}, J=1.0 \mathrm{~Hz}, 2 \mathrm{H},-$ $\mathrm{C} \underline{\mathrm{H}}=\mathrm{C} \underline{\mathrm{H}}-), 5.26$ (t, $J=0.8 \mathrm{~Hz}, 2 \mathrm{H},-\mathrm{C} \underline{\mathrm{H}}(-\mathrm{O}-) \mathrm{C}-), 3.58\left(\mathrm{t}, J=7.1 \mathrm{~Hz}, 2 \mathrm{H},-\mathrm{NC}_{2} \mathrm{CH}_{2}-\right), 2.96$ (t, $J=7.2 \mathrm{~Hz}, 2 \mathrm{H}$, $\left.-(\mathrm{O}=\mathrm{C}) \mathrm{SC}_{2} \mathrm{CH}_{2}-\right), 2.85$ (s, 2H, - $\left.\underline{\mathrm{H}}(-\mathrm{C}=\mathrm{O}) \mathrm{C} \underline{\mathrm{H}}-\right), 2.48-2.61\left(\mathrm{~m}, 32 \mathrm{H},-\underline{\mathrm{C}}_{2} \mathrm{SC}_{2}-\underline{\mathrm{H}}_{2}\right), 2.33$ (t, $J=7.4 \mathrm{~Hz}, 3 \mathrm{H}$, $\left.\mathrm{C}_{3}(\mathrm{O}=\mathrm{C}-) \mathrm{SCH}_{2} \mathrm{CH}_{2}-\right), 1.84\left(\mathrm{td}, J=7.2,5.4 \mathrm{~Hz}, 4 \mathrm{H},-\mathrm{CH}_{2} \underline{\mathrm{CH}}_{2} \mathrm{CH}_{2} \mathrm{~S}-\right), 1.55$ (pd, $J=7.9,7.5,2.5 \mathrm{~Hz}, 12 \mathrm{H},-$ $\mathrm{C}_{2} \mathrm{CH}_{2} \mathrm{CH}_{3}$ ), 1.40 (hd, $\left.J=7.3,1.6 \mathrm{~Hz}, 12 \mathrm{H},-\mathrm{CH}_{2} \underline{\mathrm{C}}_{2} \mathrm{CH}_{3}\right), 1.01\left(\mathrm{~m}, 16 \mathrm{H},-\mathrm{SiCH}_{2} \mathrm{CH}_{2}-\right), 0.92(\mathrm{~m}, 18 \mathrm{H},-$ $\left.\mathrm{CH}_{2} \mathrm{CH}_{2} \mathrm{CH}_{3}\right) .{ }^{13} \mathrm{C} \mathrm{NMR}\left(77 \mathrm{MHz}, \mathrm{CDCl}_{3}, \mathrm{ppm}, \delta\right): 195.30,176.03,136.39,80.83,47.33,37.85,31.46,31.39$, $30.54,30.42,29.07,28.51,27.90,27.12,25.78,25.73,25.55,21.92,13.64,12.91,12.87,12.83,12.72$.

p-FPOSS monomer (1.45 g, yield: 84.3\%). ${ }^{1} \mathrm{H} \mathrm{NMR}\left(400 \mathrm{MHz}, \mathrm{CDCl}_{3}, \mathrm{ppm}, \delta\right): 6.52(\mathrm{~d}, J=1.0 \mathrm{~Hz}, 2 \mathrm{H},-$ 
$\mathrm{C} \underline{\mathrm{H}}=\mathrm{C} \underline{\mathrm{H}}-), 5.26$ (t, $J=1.0 \mathrm{~Hz}, 2 \mathrm{H},-\mathrm{C} \underline{\mathrm{H}}(-\mathrm{O}-) \mathrm{C}-), 3.59$ (t, $\left.J=7.1 \mathrm{~Hz}, 2 \mathrm{H},-\mathrm{NC}_{2} \mathrm{CH}_{2}-\right), 3.11(\mathrm{~m}, 12 \mathrm{H},-$

$\left.\mathrm{SC}_{2} \mathrm{CF}_{3}\right), 2.97$ (t, $\left.J=7.2 \mathrm{~Hz}, 2 \mathrm{H}, \mathrm{CH}_{3}(\mathrm{O}=\mathrm{C}) \mathrm{SC}_{\underline{H}_{2}} \mathrm{CH}_{2}-\right), 2.85(\mathrm{~s}, 2 \mathrm{H},-\mathrm{C} \underline{\mathrm{H}}(-\mathrm{C}=\mathrm{O}) \mathrm{C} \underline{\mathrm{H}}-)$, 2.52-2.77 (m, 20H, $\left.-\underline{\mathrm{C}}_{2} \mathrm{SCH}_{2} \mathrm{CF}_{3},-\mathrm{C}_{2} \mathrm{SC}_{2} \mathrm{CH}_{2}-\right), 2.34\left(\mathrm{t}, J=7.4 \mathrm{~Hz}, 3 \mathrm{H}, \mathrm{C}_{3}(\mathrm{O}=\mathrm{C}-) \mathrm{SCH}_{2} \mathrm{CH}_{2-}\right), 1.85$ (td, $J=7.3,3.5 \mathrm{~Hz}$, 4H, $\left.-\mathrm{CH}_{2} \underline{\mathrm{C}}_{2} \mathrm{CH}_{2} \mathrm{~S}-\right), 1.05$ (m, 16H, - $\left.\mathrm{SiC}_{2} \mathrm{CH}_{2}-\right) .{ }^{13} \mathrm{C} \mathrm{NMR}\left(77 \mathrm{MHz}, \mathrm{CDCl}_{3}, \mathrm{ppm}, \delta\right): 195.99,176.22$ $136.47,129.30,127.10,124.89,122.71,80.93,47.38,37.92,30.57,30.54,29.17,28.68,27.92,27.18,25.61$, $25.57,25.42,12.60,12.48,12.26$.

p-PPOSS monomer (1.46 g, yield: 82.5\%). ${ }^{1} \mathrm{H}$ NMR (400 MHz, $\left.\mathrm{CDCl}_{3}, \mathrm{ppm}, \delta\right)$ : 7.23-7.34 (m, 30H, $\left.\mathrm{SCH}_{2} \underline{\mathrm{Ph}}\right), 6.48$ (d, $\left.J=1.0 \mathrm{~Hz}, 2 \mathrm{H},-\mathrm{C} \underline{\mathrm{H}}=\mathrm{C} \underline{\mathrm{H}}-\right), 5.25$ (t, $\left.J=1.0 \mathrm{~Hz}, 2 \mathrm{H},-\mathrm{C} \underline{\mathrm{H}}(-\mathrm{O}-) \mathrm{C}-\right), 3.70$ (d, $J=5.1 \mathrm{~Hz}, 12 \mathrm{H}$, $\left.-\mathrm{SC} \underline{H}_{2} \mathrm{Ph}\right), 3.59\left(\mathrm{t}, J=7.1 \mathrm{~Hz}, 2 \mathrm{H},-\mathrm{NC}_{2} \mathrm{CH}_{2}-\right), 2.97$ (t, $\left.J=7.2 \mathrm{~Hz}, 2 \mathrm{H}, \mathrm{CH}_{3}(\mathrm{O}=\mathrm{C}) \mathrm{SC}_{2} \mathrm{CH}_{2}-\right), 2.82(\mathrm{~s}, 2 \mathrm{H}$, $-\mathrm{C} \underline{\mathrm{H}}(-\mathrm{C}=\mathrm{O}) \mathrm{C} \underline{\mathrm{H}}-), 2.41-2.58\left(\mathrm{~m}, 20 \mathrm{H},-\underline{\mathrm{C}}_{2} \mathrm{SCH}_{2} \mathrm{Ph},-\underline{\mathrm{C}}_{2} \mathrm{SC}_{2} \mathrm{CH}_{2}-\right), 2.33\left(\mathrm{~s}, 3 \mathrm{H}, \mathrm{C}_{3}(\mathrm{O}=\mathrm{C}-) \mathrm{SCH}_{2} \mathrm{CH}_{2}-\right)$, $1.84\left(\mathrm{td}, J=7.3,3.3 \mathrm{~Hz}, 4 \mathrm{H},-\mathrm{CH}_{2} \mathrm{C}_{2} \mathrm{CH}_{2} \mathrm{~S}-\right), 1.04$ (m, 16H, $\left.-\mathrm{SiC}_{2} \mathrm{CH}_{2}-\right) .{ }^{13} \mathrm{C} \mathrm{NMR}\left(77 \mathrm{MHz}, \mathrm{CDCl}_{3}, \mathrm{ppm}\right.$, $\delta): 195.36,176.05,138.21,136.34,128.65,128.39,126.88,80.79,47.28,37.86,35.78,30.55,30.41,29.07$, $25.52,27.91,27.13,25.67,25.49,24.98,24.94,12.73,12.62,12.42$.

\subsection{Discrete Homo Polymeric Chains}

Each IEG cycle consists of two steps of deprotection (Mal-R $\mathbf{R}_{n}$ and $\left.\mathbf{R}_{\boldsymbol{m}}-\mathbf{S H}\right)$ and one coupling reaction $\left(\mathbf{R}_{n+m}\right)$.

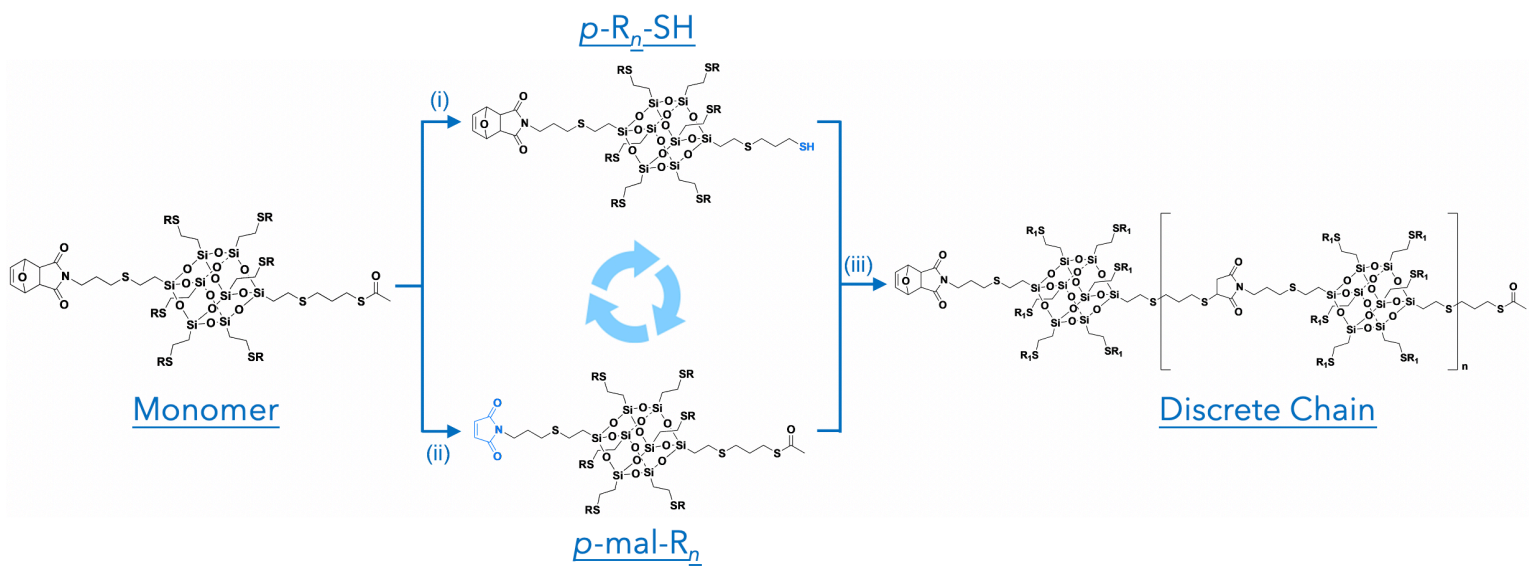

Scheme S2. iterative exponential growth of discrete POSS chains: (i) Acetyl chloride, $-78{ }^{\circ} \mathrm{C}, \mathrm{CH}_{2} \mathrm{Cl}_{2} / \mathrm{MeOH}$, $7 \mathrm{~h}$; (ii) Toluene, $110^{\circ} \mathrm{C}, 5 \mathrm{~h}$; (iii) $\mathrm{TEA}, \mathrm{CHCl}_{3}, 25^{\circ} \mathrm{C}, 8 \mathrm{~h}$. 


\subsubsection{Dimer: $p$ - $\mathbf{R}_{2}$}

p-Mal-R R $_{1}$ monomer $\left(1.0 \mathrm{mmol}\right.$, corresponding to $1.56 \mathrm{~g}$ of $p-\mathrm{C}_{1}, 1.72 \mathrm{~g}$ of $p-\mathrm{F}_{1}$, or $1.77 \mathrm{~g}$ of $p$ - $\left.\mathrm{P}_{1}\right)$ was dissolved in $30 \mathrm{~mL}$ of toluene in a $100 \mathrm{~mL}$ three-neck flask equipped with a condenser. After stirring and refluxing at $120^{\circ} \mathrm{C}$ under argon flow for about $6 \mathrm{~h}$, TLC indicated the reaction was complete. Toluene was removed under vacuum. The residue was dried under vacuum at $25^{\circ} \mathrm{C}$ for $24 \mathrm{~h}$ to afford $\boldsymbol{p}$-Mal-R $\mathbf{1}$ as colorless oil (p-Mal-C1: 1.47 g, yield: 98\%; p-Mal-F1:1.60g, yield: 97\%; p-Mal-P1: 1.67g, yield: 98\%).

p-R-SH: monomer (1.0 mmol, corresponding to $1.56 \mathrm{~g}$ of $p-\mathrm{C}_{1}, 1.72 \mathrm{~g}$ of $p-\mathrm{F}_{1}$, or $1.77 \mathrm{~g}$ of $p$ - $\left.\mathrm{P}_{1}\right)$ was dissolved in $10 \mathrm{~mL}$ of $\mathrm{MeOH}$ and $30 \mathrm{~mL}$ of $\mathrm{CH}_{2} \mathrm{Cl}_{2}$ in a $100 \mathrm{~mL}$ two-neck round-bottom flask. The mixture was cooled to $-78^{\circ} \mathrm{C}$. Acetyl chloride $(15.0 \mathrm{~mL}, 0.11 \mathrm{~mol})$ was then added dropwise to the solution. The mixture was warmed to room temperature and further stirred for $12 \mathrm{~h}$. The reaction mixture was quenched with $20 \mathrm{~mL}$ water and washed with $30 \mathrm{~mL}$ saturated $\mathrm{NaHCO}_{3}$ (aq.). The organic layer was combined and washed with $30 \mathrm{~mL}$ of water and dried with anhydrous $\mathrm{Na}_{2} \mathrm{SO}_{4}$. DCM was removed under vacuum to afford p-R1-SH as colorless oil liquid (p-C1-SH: 1.43 g, yield: 94\%; p-F1-SH:1.53 g, yield: 91\%; p-P1-SH: 1.60 g, yield: 93\%).

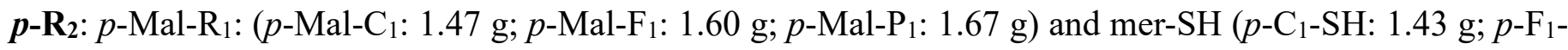
SH: $1.53 \mathrm{~g}$; $p$ - $\mathrm{P}_{1}-\mathrm{SH}: 1.60 \mathrm{~g}$ ) were dissolved in $25 \mathrm{~mL}$ of anhydrous $\mathrm{CHCl}_{3}$ in a $50 \mathrm{~mL}$ two-neck round-bottom flask equipped with a $25 \mathrm{~mL}$ slow-addition apparatus under argon atmosphere at $25^{\circ} \mathrm{C}$. TEA $(1.0 \mathrm{~mL}, 7.3$ mmol) was added dropwise to the solution and the mixture was stirred for $24 \mathrm{~h}$. The reaction mixture was quenched with $20 \mathrm{~mL}$ of water and washed with $30 \mathrm{~mL}$ of saturated $\mathrm{NaHCO}_{3}$ (aq.). The combined organic layer was dried with anhydrous $\mathrm{Na}_{2} \mathrm{SO}_{4}$ and the solvent was removed to afford the crude product, which was further purified by column chromatography with PE/EA (4/1) as eluent to give the $\boldsymbol{p}$ - $\mathbf{R}_{\mathbf{2}}$ as colorless oil liquid. p-C $\mathbf{C}_{2}\left(2.00\right.$ g, yield: 69\%). ${ }^{1} \mathrm{H}$ NMR (400 MHz, $\left.\mathrm{CDCl}_{3}, \mathrm{ppm}, \delta\right): 6.51$ (s, 2H, $\left.-\mathrm{C} \underline{\mathrm{H}}=\mathrm{C} \underline{\mathrm{H}}-\right)$ ), 5.26 (t, $J=1.0 \mathrm{~Hz}$, 
2H, -Cㅌ(-O-)C-), 3.73 (dd, $\left.J=9.0,3.7 \mathrm{~Hz}, 1 \mathrm{H},-\mathrm{SC} \underline{\mathrm{H}}(\mathrm{C}=\mathrm{O}) \mathrm{CH}_{2}-\right), 3.59$ (m, 4H, - $\left.\mathrm{NC}_{2} \mathrm{CH}_{2}-\right), 3.14,3.05$ (m,

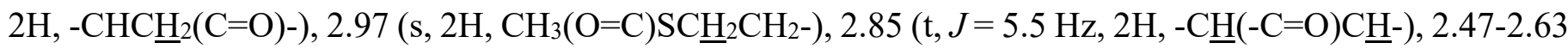
(m, 66H, - $\left.\underline{\mathrm{CH}}_{2} \mathrm{SC} \underline{\mathrm{H}}_{2}-,-\underline{\mathrm{C}}_{2} \mathrm{SCH}-\right), 2.34$ (s, 3H, $\left.\mathrm{CH}_{3}(\mathrm{O}=\mathrm{C}-) \mathrm{SCH}_{2} \mathrm{CH}_{2}-\right), 1.86\left(\mathrm{~m}, 8 \mathrm{H},-\mathrm{CH}_{2} \mathrm{C}_{2} \mathrm{CH}_{2} \mathrm{~S}-\right), 1.56$ (m, 24H, $\left.-\mathrm{C}_{2} \mathrm{CH}_{2} \mathrm{CH}_{3}\right), 1.42\left(\mathrm{~m}, 24 \mathrm{H},-\mathrm{CH}_{2} \mathrm{C}_{2} \mathrm{CH}_{3}\right), 1.02\left(\mathrm{~m}, 32 \mathrm{H},-\mathrm{SiC}_{2} \mathrm{CH}_{2}-\right), 0.93(\mathrm{t}, J=7.3 \mathrm{~Hz}, 36 \mathrm{H}$, $\left.-\mathrm{CH}_{2} \mathrm{CH}_{2} \underline{\mathrm{CH}}_{3}\right) .{ }^{13} \mathrm{C} \mathrm{NMR}\left(77 \mathrm{MHz}, \mathrm{CDCl}_{3}, \mathrm{ppm}, \delta\right): 194.93,176.12,175.81,174.20,136.20,80.65,47.15$, $38.65,37.83,37.63,35.73,31.27,31.17,30.36,30.33,30.20,30.12,28.90,28.56,28.27,27.70,26.93,26.80$, $25.56,25.49,25.29,21.73,13.47,12.68,12.53$.

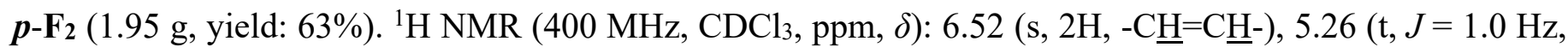
2H, -Cㅡ(-O-)C-), 3.75 (d, $\left.J=5.6 \mathrm{~Hz}, 1 \mathrm{H},-\mathrm{SC} \underline{\mathrm{H}}(\mathrm{C}=\mathrm{O}) \mathrm{CH}_{2}-\right), 3.59\left(\mathrm{dt}, J=14.2,7.1 \mathrm{~Hz}, 4 \mathrm{H},-\mathrm{NC}_{2} \mathrm{CH}_{2}-\right)$, 3.04-3.10 (m, 26H, -SC $\left.\underline{\mathrm{H}}_{2} \mathrm{CF}_{3},-\mathrm{CHC}_{2}(\mathrm{C}=\mathrm{O})-\right), 2.97$ (t, $\left.J=5.1 \mathrm{~Hz}, 2 \mathrm{H}, \mathrm{CH}_{3}(\mathrm{O}=\mathrm{C}) \mathrm{SC}_{2} \mathrm{CH}_{2}-\right), 2.85(\mathrm{~s}, 2 \mathrm{H}$, $-\mathrm{C} \underline{\mathrm{H}}(-\mathrm{C}=\mathrm{O}) \mathrm{C} \underline{\mathrm{H}}-), \quad 2.50-2.77 \quad\left(\mathrm{~m}, \quad 42 \mathrm{H}, \quad-\mathrm{C}_{2} \mathrm{SCH}_{2} \mathrm{CF}_{3},-\mathrm{C}_{2} \mathrm{SC}_{2} \mathrm{CH}_{2}-, \quad-\mathrm{C}_{2} 2 \mathrm{SCH}-\right), \quad 2.34 \quad(\mathrm{~s}, \quad 3 \mathrm{H}$, $\left.\mathrm{C}_{3}(\mathrm{O}=\mathrm{C}-) \mathrm{SC}-\right), 1.86\left(\mathrm{~m}, 8 \mathrm{H},-\mathrm{CH}_{2} \underline{\mathrm{CH}}_{2} \mathrm{CH}_{2} \mathrm{~S}-\right), 1.05$ (ddt, $J=10.8,8.0,3.9 \mathrm{~Hz}, 32 \mathrm{H},-\mathrm{SiC}_{2} \mathrm{CH}_{2}-$ ).

p-P2 (2.16 g, yield: 66\%). ${ }^{1} \mathrm{H}$ NMR (400 MHz, $\left.\mathrm{CDCl}_{3}, \mathrm{ppm}, \delta\right): 7.25-7.34$ (m, 60H, $\left.-\mathrm{SCH}_{2} \underline{\mathrm{Ph}}\right), 6.48(\mathrm{~s}, 2 \mathrm{H},-$ $\mathrm{C} \underline{\mathrm{H}}=\mathrm{C} \underline{\mathrm{H}}-), 5.24(\mathrm{t}, J=1.0 \mathrm{~Hz}, 2 \mathrm{H},-\mathrm{C} \underline{\mathrm{H}}(-\mathrm{O}-) \mathrm{C}-), 3.70-3.74\left(\mathrm{~m}, 25 \mathrm{H},-\mathrm{SC} \underline{H}_{2} \mathrm{Ph},-\mathrm{SC} \underline{\mathrm{H}}(\mathrm{C}=\mathrm{O}) \mathrm{CH}_{2}-\right), 3.58(\mathrm{q}, J$ $\left.=7.9,7.4 \mathrm{~Hz}, 4 \mathrm{H},-\mathrm{NC}_{2} \mathrm{CH}_{2}-\right), 3.1,3.14\left(\mathrm{~m}, 2 \mathrm{H},-\mathrm{CHCH}_{2}(\mathrm{C}=\mathrm{O})-\right), 2.97(\mathrm{t}, J=5.3 \mathrm{~Hz}, 2 \mathrm{H}$, $\left.\mathrm{CH}_{3}(\mathrm{O}=\mathrm{C}) \mathrm{SC}_{2} \mathrm{CH}_{2}-\right), 2.81(\mathrm{~m}, 2 \mathrm{H},-\mathrm{C} \underline{\mathrm{H}}(-\mathrm{C}=\mathrm{O}) \mathrm{C} \underline{\mathrm{H}}-), 2.41-2.63\left(\mathrm{~m}, 42 \mathrm{H},-\underline{\mathrm{C}}_{2} \mathrm{SCH}_{2} \mathrm{Ph},-\underline{\mathrm{C}}_{2} \mathrm{SC}_{2} \mathrm{CH}_{2}-,-\right.$ $\left.\mathrm{C}_{2} \mathrm{SCH}-\right), 2.32\left(\mathrm{~s}, 3 \mathrm{H}, \mathrm{CH}_{3}(\mathrm{O}=\mathrm{C}-) \mathrm{SCH}_{2} \mathrm{CH}_{2}-\right), 1.85$ (m, 8H, $\left.-\mathrm{CH}_{2} \underline{\mathrm{C}}_{2} \mathrm{CH}_{2} \mathrm{~S}-\right), 0.96$ (q, J = 9.3 Hz, 32H, $\mathrm{SiC}_{2} \mathrm{CH}_{2}-$ ).

\subsubsection{4mer: $p-\mathrm{R}_{4}$}

p-Mal-R $2: p-\mathrm{R}_{2}\left(0.3 \mathrm{mmol}, p-\mathrm{C}_{2}: 0.91 \mathrm{~g} ; p-\mathrm{F}_{2}: 1.00 \mathrm{~g} ; p-\mathrm{P}_{2}: 1.03 \mathrm{~g}\right)$ was dissolved in $20 \mathrm{~mL}$ of toluene in a 50 $\mathrm{mL}$ three-neck flask equipped with a condenser. The mixture was stirred and refluxed at $120^{\circ} \mathrm{C}$ under argon flow for about $6 \mathrm{~h}$. TLC showed the reaction was complete. After cooling to room temperature, toluene was 
evaporated under vacuum. The residue was dried under vacuum at $25{ }^{\circ} \mathrm{C}$ for $24 \mathrm{~h}$ to afford $\boldsymbol{p}$-Mal-R $\mathbf{R}_{\mathbf{2}}$ as colorless oil liquids (p-Mal-C2: 0.81 g, yield: 92\%; $\boldsymbol{p}$-Mal-F2: 0.90 g, yield: 91\%; $\boldsymbol{p}$-Mal-P2: 0.95 g, yield: $94 \%)$.

p-R2-SH: $p-\mathrm{R}_{2}\left(0.3 \mathrm{mmol}, p-\mathrm{C}_{2}: 0.91 \mathrm{~g} ; p-\mathrm{F}_{2}: 1.00 \mathrm{~g} ; p-\mathrm{P}_{2}: 1.03 \mathrm{~g}\right)$ was dissolved in $6 \mathrm{~mL}$ of $\mathrm{MeOH}$ and 15 $\mathrm{mL}$ of $\mathrm{CH}_{2} \mathrm{Cl}_{2}$ in a $50 \mathrm{~mL}$ two-neck round-bottom flask. The mixture was cooled to $-78{ }^{\circ} \mathrm{C}$. Acetyl chloride $(8.0 \mathrm{~mL}, 59 \mathrm{mmol})$ was then added dropwise to the solution. The mixture was warmed to room temperature and stirred for $12 \mathrm{~h}$. The reaction mixture was quenched with $20 \mathrm{~mL}$ of water and washed with $30 \mathrm{~mL}$ of saturated $\mathrm{NaHCO}_{3}$ (aq.). The organic layer was combined and washed with $30 \mathrm{~mL}$ of water and dried with anhydrous $\mathrm{Na}_{2} \mathrm{SO}_{4}$. DCM was removed under vacuum to afford $\boldsymbol{p}$ - $\mathbf{R}_{\mathbf{2}}-\mathbf{S H}$ as colorless oil liquid $\left(\boldsymbol{p}\right.$ - $\mathbf{C}_{2}-\mathbf{S H}$ : 0.79 g, yield: 89\%; p-F2-SH: 0.87 g, yield: $88 \%$; $\boldsymbol{p}$-P2-SH: 0.90 g, yield: $90 \%$ ).

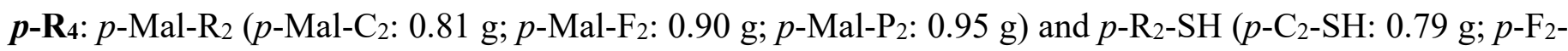
SH: $0.87 \mathrm{~g}$; $p$ - $\mathrm{P}_{2}-\mathrm{SH}: 0.90 \mathrm{~g}$ ) were dissolved in $20 \mathrm{~mL}$ of anhydrous $\mathrm{CHCl}_{3}$ in a $50 \mathrm{~mL}$ two-neck round-bottom flask equipped with a $25 \mathrm{~mL}$ slow-addition apparatus under argon atmosphere at $25{ }^{\circ} \mathrm{C}$. TEA $(0.8 \mathrm{~mL}, 5.8$ mmol) was added dropwise to the solution and the mixture was stirred for about $24 \mathrm{~h}$. The reaction mixture was washed with $30 \mathrm{~mL}$ of saturated $\mathrm{NaHCO}_{3}$ (aq.). The combined organic layer was dried with anhydrous $\mathrm{Na}_{2} \mathrm{SO}_{4}$ and the solvent was evaporated to afford the crude product, which was purified by recycling preparative SEC to give the $\boldsymbol{p}-\mathbf{R}_{\mathbf{4}}$ as colorless oil liquid.

p-C 4 (0.88 g, yield: 55\%). ${ }^{1} \mathrm{H}$ NMR (400 MHz, $\left.\mathrm{CDCl}_{3}, \mathrm{ppm}, \delta\right): 6.52(\mathrm{~s}, 2 \mathrm{H},-\mathrm{C} \underline{\mathrm{H}}=\mathrm{C} \underline{\mathrm{H}}-), 5.26(\mathrm{t}, J=1.0 \mathrm{~Hz}$, $2 \mathrm{H},-\mathrm{C} \underline{\mathrm{H}}(-\mathrm{O}-) \mathrm{C}-), 3.74\left(\mathrm{dd}, J=9.0,3.6 \mathrm{~Hz}, 3 \mathrm{H},-\mathrm{SC} \underline{\mathrm{H}}(\mathrm{C}=\mathrm{O}) \mathrm{CH}_{2}-\right), 3.58\left(\mathrm{dt}, J=13.6,7.2 \mathrm{~Hz}, 8 \mathrm{H},-\mathrm{NC}_{2} \mathrm{CH}_{2}-\right)$, 3.14, $3.05\left(\mathrm{~m}, 6 \mathrm{H},-\mathrm{CHCH}_{2}(\mathrm{C}=\mathrm{O})-\right), 2.97\left(\mathrm{t}, J=7.2 \mathrm{~Hz}, 2 \mathrm{H}, \mathrm{CH}_{3}(\mathrm{O}=\mathrm{C}) \mathrm{SC}_{2} \mathrm{CH}_{2}-\right), 2.84(\mathrm{~s}, 2 \mathrm{H},-\mathrm{C} \underline{\mathrm{H}}(-$ $\mathrm{C}=\mathrm{O}) \mathrm{C} \underline{\mathrm{H}}-)$, 2.44-2.63 (m, 134H, $\left.-\underline{\mathrm{C}}_{2} \mathrm{SC}_{2}-{ }_{2},-\underline{\mathrm{H}}_{2} \mathrm{SCH}-\right), 2.36$ (s, 3H, $\left.\underline{\mathrm{H}}_{3}(\mathrm{O}=\mathrm{C}-) \mathrm{SCH}_{2} \mathrm{CH}_{2}-\right), 1.85(\mathrm{~m}, 16 \mathrm{H}$, $\left.-\mathrm{CH}_{2} \underline{\mathrm{CH}}_{2} \mathrm{CH}_{2} \mathrm{~S}-\right), 1.57$ (m, 48H, - $\left.\underline{\mathrm{CH}}_{2} \mathrm{CH}_{2} \mathrm{CH}_{3}\right), 1.42\left(\mathrm{~m}, 48 \mathrm{H},-\mathrm{CH}_{2} \mathrm{CH}_{2} \mathrm{CH}_{3}\right), 1.03\left(\mathrm{~m}, 64 \mathrm{H},-\mathrm{SiCH}_{2} \mathrm{CH}_{2}-\right)$, 
$0.94\left(\mathrm{t}, J=7.9 \mathrm{~Hz}, 72 \mathrm{H},-\mathrm{CH}_{2} \mathrm{CH}_{2} \mathrm{C}_{3}\right)$.

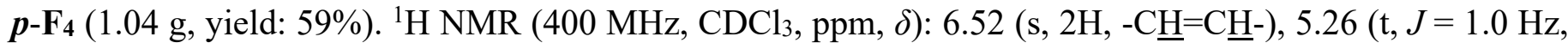

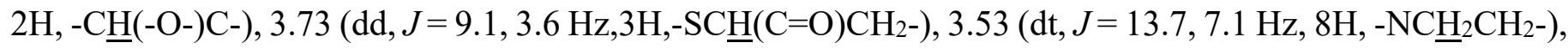
3.04-3.10 (m, 54H, - $\left.\mathrm{SC}_{2} \mathrm{CF}_{3},-\mathrm{CHC}_{2}(\mathrm{C}=\mathrm{O})-\right), 2.97$ (t, $\left.J=7.6 \mathrm{~Hz}, 2 \mathrm{H}, \mathrm{CH}_{3}(\mathrm{O}=\mathrm{C}) \mathrm{SC}_{2} \mathrm{CH}_{2}-\right), 2.86(\mathrm{~s}, 2 \mathrm{H}$, $-\mathrm{C} \underline{\mathrm{H}}(-\mathrm{C}=\mathrm{O}) \mathrm{C} \underline{\mathrm{H}}-), \quad 2.50-2.77\left(\mathrm{~m}, \quad 86 \mathrm{H}, \quad-\mathrm{C}_{2} \mathrm{SCH}_{2} \mathrm{CF}_{3}, \quad-\mathrm{C}_{2} \mathrm{SC}_{2} \mathrm{CH}_{2}-, \quad-\mathrm{C}_{2} \mathrm{SCH}-\right), \quad 2.37 \quad(\mathrm{~s}, \quad 3 \mathrm{H}$, $\left.\mathrm{C}_{3}(\mathrm{O}=\mathrm{C}-) \mathrm{SC}-\right), 1.82\left(\mathrm{~m}, 16 \mathrm{H},-\mathrm{CH}_{2} \underline{\mathrm{C}}_{2} \mathrm{CH}_{2} \mathrm{~S}-\right), 1.05$ (m, 64H, - $\left.\mathrm{SiC}_{2} \mathrm{CH}_{2}-\right)$.

p-P4 (1.00 g, yield: 54\%). ${ }^{1} \mathrm{H}$ NMR (400 MHz, $\left.\mathrm{CDCl}_{3}, \mathrm{ppm}, \delta\right): 7.26-7.34$ (m, 120H, $-\mathrm{SCH}_{2} \underline{\mathrm{Ph}}$ ), 6.48 (s, 2H, $-\mathrm{C} \underline{\mathrm{H}}=\mathrm{C} \underline{\mathrm{H}}-), 5.23$ (t, $J=1.0 \mathrm{~Hz}, 2 \mathrm{H},-\mathrm{C} \underline{\mathrm{H}}(-\mathrm{O}-) \mathrm{C}-), 3.70-3.75\left(\mathrm{~m}, 51 \mathrm{H},-\mathrm{SC}_{2} 2 \mathrm{Ph},-\mathrm{SC} \underline{\mathrm{H}}(\mathrm{C}=\mathrm{O}) \mathrm{CH}_{2}-\right), 3.58$ (dt, $\left.J=13.1,7.9 \mathrm{~Hz}, 8 \mathrm{H},-\mathrm{NC}_{2} \mathrm{CH}_{2}-\right), 3.11,3.14$ (m, 6H, $\left.-\mathrm{CHC}_{2}(\mathrm{C}=\mathrm{O})-\right), 2.93$ (t, $J=7.3 \mathrm{~Hz}, 2 \mathrm{H}$, $\left.\mathrm{CH}_{3}(\mathrm{O}=\mathrm{C}) \mathrm{SC}_{2} \mathrm{CH}_{2}-\right), 2.84(\mathrm{~s}, 2 \mathrm{H},-\mathrm{C} \underline{\mathrm{H}}(-\mathrm{C}=\mathrm{O}) \mathrm{C} \underline{\mathrm{H}}-), 2.41-2.63\left(\mathrm{~m}, 86 \mathrm{H},-\underline{\mathrm{C}}_{2} \mathrm{SCH}_{2} \mathrm{Ph},-\underline{\mathrm{C}}_{2} \mathrm{SC}_{2} \mathrm{CH}_{2}-,-\right.$

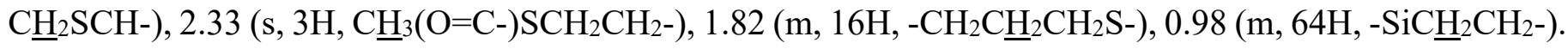

\subsubsection{8mer: $p-\mathrm{R}_{8}$}

p-Mal-R4: $p$-R 4 (0.07 mmol, $p$-C 4 : $0.41 \mathrm{~g} ; p$ - $\left.\mathrm{P}_{4}: 0.47 \mathrm{~g}\right)$ was dissolved in $15 \mathrm{~mL}$ of toluene in a $50 \mathrm{~mL}$ threeneck flask equipped with a condenser. The mixture was stirred and refluxed at $120^{\circ} \mathrm{C}$ under argon flow for about $6 \mathrm{~h}$. TLC showed the reaction was complete. After cooling to room temperature, toluene was evaporated under vacuum. The residue was dried under vacuum at $25^{\circ} \mathrm{C}$ for $24 \mathrm{~h}$ to afford $\boldsymbol{p}$-Mal-R $\mathbf{4}$ as colorless oil liquid (p-Mal-C4: 0.37 g, yield: 90\%; p-Mal-P4: 0.39 g, yield: 89\%).

p-R4-SH: $p$ - $\mathrm{R}_{4}\left(0.07 \mathrm{mmol}, p-\mathrm{C}_{4}: 0.41 \mathrm{~g} ; p\right.$ - $\left.\mathrm{P}_{4}: 0.47 \mathrm{~g}\right)$ was dissolved in $6 \mathrm{~mL}$ of $\mathrm{MeOH}$ and $22 \mathrm{~mL}$ of $\mathrm{CH}_{2} \mathrm{Cl}_{2}$ in a $50 \mathrm{~mL}$ two-neck round-bottom flask equipped with a condenser. The mixture was cooled to $-78^{\circ} \mathrm{C}$. Acetyl chloride $(8.0 \mathrm{~mL}, 59 \mathrm{mmol})$ was then added dropwise to the solution. The mixture was warmed to room temperature and stirred for $12 \mathrm{~h}$. The reaction mixture was quenched with $20 \mathrm{~mL}$ of water and washed with $30 \mathrm{~mL}$ of saturated $\mathrm{NaHCO}_{3}$ (aq.). The organic layer was combined and washed with $30 \mathrm{~mL}$ of water and 
dried with anhydrous $\mathrm{Na}_{2} \mathrm{SO}_{4}$. DCM was concentrated under vacuum to afford $\boldsymbol{p}$ - $\mathbf{R}_{\mathbf{4}}-\mathbf{S H}$ as colorless oil liquid (p-C4-SH: 0.35 g, yield: 86\%; p-P4-SH: 0.38 g, yield: 88\%).

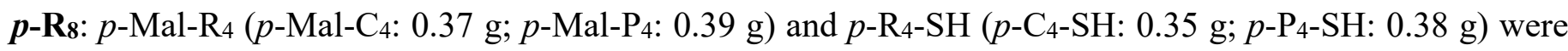
dissolved in $15 \mathrm{~mL}$ of anhydrous $\mathrm{CHCl}_{3}$ in a $25 \mathrm{~mL}$ two-neck round-bottom flask equipped with a $5 \mathrm{~mL}$ slowaddition apparatus under argon atmosphere at $25{ }^{\circ} \mathrm{C}$. TEA $(0.2 \mathrm{~mL}, 1.5 \mathrm{mmol})$ was added dropwise to the solution and the mixture was stirred for about $24 \mathrm{~h}$. The reaction mixture was quenched with $20 \mathrm{~mL}$ water and washed with $30 \mathrm{~mL}$ saturated $\mathrm{NaHCO}_{3}$ (aq.). The combined organic layer was dried with anhydrous $\mathrm{Na}_{2} \mathrm{SO}_{4}$ and the solvent was evaporated to afford the crude product which was further purified by recycling preparative SEC to give the $\boldsymbol{p}$ - $\mathbf{R}_{\mathbf{8}}$ as colorless oil liquid.

p-C8 (0.32 g, yield: 45\%). ${ }^{1} \mathrm{H}$ NMR (400 MHz, $\left.\mathrm{CDCl}_{3}, \mathrm{ppm}, \delta\right): 6.51(\mathrm{~s}, 2 \mathrm{H},-\mathrm{C} \underline{\mathrm{H}}=\mathrm{C} \underline{\mathrm{H}}-), 5.26(\mathrm{t}, J=1.0 \mathrm{~Hz}$, 2H, -C프(-O-)C-), 3.74 (dd, $\left.J=9.5,3.1 \mathrm{~Hz}, 7 \mathrm{H},-\mathrm{SC} \underline{\mathrm{H}}(\mathrm{C}=\mathrm{O}) \mathrm{CH}_{2}-\right), 3.55$ (dt, $J=13.1,7.6 \mathrm{~Hz}, 16 \mathrm{H},-$ $\left.\mathrm{NC}_{2} \mathrm{CH}_{2}-\right), 3.14,3.05\left(\mathrm{~m}, 14 \mathrm{H},-\mathrm{CHC}_{2}(\mathrm{C}=\mathrm{O})-\right), 2.94\left(\mathrm{t}, J=7.0 \mathrm{~Hz}, 2 \mathrm{H}, \mathrm{CH}_{3}(\mathrm{O}=\mathrm{C}) \mathrm{SC}_{2} \mathrm{CH}_{2}-\right), 2.84(\mathrm{~s}$, 2H, - $\mathrm{C} \underline{\mathrm{H}}(-\mathrm{C}=\mathrm{O}) \mathrm{C} \underline{\mathrm{H}}-), 2.47-2.63\left(\mathrm{~m}, 270 \mathrm{H},-\underline{\mathrm{C}}_{2} \mathrm{SC}_{2}{ }_{2}-,-\underline{\mathrm{H}}_{2} \mathrm{SCH}-\right), 2.34\left(\mathrm{~s}, 3 \mathrm{H}, \mathrm{C}_{3}(\mathrm{O}=\mathrm{C}-) \mathrm{SCH}_{2} \mathrm{CH}_{2}-\right), 1.85$ (m, 32H, $\left.-\mathrm{CH}_{2} \underline{\mathrm{C}}_{2} \mathrm{CH}_{2} \mathrm{~S}-\right), 1.53\left(\mathrm{~m}, 96 \mathrm{H},-\mathrm{C}_{2} \mathrm{CH}_{2} \mathrm{CH}_{3}\right), 1.44\left(\mathrm{~m}, 96 \mathrm{H},-\mathrm{CH}_{2} \underline{\mathrm{C}}_{2} \mathrm{CH}_{3}\right), 1.03(\mathrm{~m}, 128 \mathrm{H},-$ $\left.\mathrm{SiC}_{2} \mathrm{CH}_{2}-\right), 0.94\left(\mathrm{~m}, 144 \mathrm{H},-\mathrm{CH}_{2} \mathrm{CH}_{2} \underline{\mathrm{C}}_{3}\right)$.

p-P8 (0.42 g, yield: 54\%). ${ }^{1} \mathrm{H}$ NMR (400 MHz, $\left.\mathrm{CDCl}_{3}, \mathrm{ppm}, \delta\right): 7.25-7.34$ (m, 240H, $-\mathrm{SCH}_{2} \underline{\mathrm{Ph}}$ ), 6.48 (s, 2H, $-\mathrm{C} \underline{\mathrm{H}}=\mathrm{C} \underline{\mathrm{H}}-), 5.25$ (t, $J=1.0 \mathrm{~Hz}, 2 \mathrm{H},-\mathrm{C} \underline{\mathrm{H}}(-\mathrm{O}-) \mathrm{C}-), 3.71-3.74\left(\mathrm{~m}, 103 \mathrm{H},-\mathrm{SC} \underline{\mathrm{H}} 2 \mathrm{Ph},-\mathrm{SC} \underline{\mathrm{H}}(\mathrm{C}=\mathrm{O}) \mathrm{CH}_{2}-\right), 3.55$ (dt, $\left.J=13.1,7.9 \mathrm{~Hz}, 16 \mathrm{H},-\mathrm{NC}_{2} \mathrm{CH}_{2}-\right), 3.11,3.14$ (m, 14H, $\left.-\mathrm{CHC}_{2}(\mathrm{C}=\mathrm{O})-\right), 2.99$ (t, $J=7.5 \mathrm{~Hz}, 2 \mathrm{H}$, $\left.\mathrm{CH}_{3}(\mathrm{O}=\mathrm{C}) \mathrm{SC}_{2} \mathrm{CH}_{2}-\right), 2.82(\mathrm{~s}, 2 \mathrm{H},-\mathrm{C} \underline{\mathrm{H}}(-\mathrm{C}=\mathrm{O}) \mathrm{C} \underline{\mathrm{H}}-), 2.41-2.63\left(\mathrm{~m}, 174 \mathrm{H},-\underline{\mathrm{C}}_{2} \mathrm{SCH}_{2} \mathrm{Ph},-\underline{\mathrm{C}}_{2} \mathrm{SC}_{2} \mathrm{CH}_{2}-\right)$, 2.33 (s, 3H, $\left.\underline{\mathrm{H}}_{3}(\mathrm{O}=\mathrm{C}-) \mathrm{SCH}_{2} \mathrm{CH}_{2}-\right), 1.84\left(\mathrm{~m}, 32 \mathrm{H},-\mathrm{CH}_{2} \mathrm{CH}_{2} \mathrm{CH}_{2} \mathrm{~S}-\right), 0.97$ (m, 128H, - $\left.\mathrm{SiC}_{2} \mathrm{CH}_{2}-\right)$.

\subsubsection{6mer: $p$-R 16}

p-Mal-R8: $p$ - $\mathrm{R}_{8}\left(8.5 \times 10^{-3} \mathrm{mmol}, p\right.$-C $\left.8: 0.10 \mathrm{~g}\right)$ was dissolved in $80 \mathrm{~mL}$ of toluene in a $25 \mathrm{~mL}$ three-neck flask 
equipped with a condenser. The mixture was stirred and refluxed at $120^{\circ} \mathrm{C}$ under argon flow for about $6 \mathrm{~h}$. TLC showed the reaction was complete. After cooling to room temperature, toluene was evaporated under vacuum. The residue was dried under vacuum at $25^{\circ} \mathrm{C}$ for $24 \mathrm{~h}$ to afford $\boldsymbol{p}$-Mal-R8 as colorless oil liquid ( $\boldsymbol{p}$ C8-mal: 78 mg, yield: 78\%).

p-R-SH: $p$ - $\mathrm{R}_{8}\left(8.5 \times 10^{-3} \mathrm{mmol}, p-\mathrm{C}_{8}: 0.10 \mathrm{~g}\right)$ was dissolved in $2 \mathrm{~mL}$ of $\mathrm{MeOH}$ and $8 \mathrm{~mL}$ of $\mathrm{CH}_{2} \mathrm{Cl}_{2}$ in a 25 $\mathrm{mL}$ two-neck round-bottom flask equipped with a condenser. The mixture was cooled to $-78{ }^{\circ} \mathrm{C}$. Acetyl chloride $(1.0 \mathrm{~mL}, 7 \mathrm{mmol})$ was then added dropwise to the solution. The mixture was warmed to room temperature and stirred for $12 \mathrm{~h}$. The reaction mixture was quenched with $20 \mathrm{~mL}$ of water and washed with $30 \mathrm{~mL}$ of saturated $\mathrm{NaHCO}_{3}$ (aq.). The organic layer was combined and washed with $30 \mathrm{~mL}$ of water and dried with anhydrous $\mathrm{Na}_{2} \mathrm{SO}_{4}$. DCM was removed under vacuum to afford $\boldsymbol{p}$ - $\mathbf{R}_{\mathbf{8}}-\mathbf{S H}$ as colorless oil liquid ( $\boldsymbol{p}$ C8-SH: $71 \mathrm{mg}$, yield: 71\%).

p-R16: p-Mal-R8 (p-Mal-C8: $78 \mathrm{mg}$ ) and p-Re-SH (p-C8-SH: $71 \mathrm{mg}$ ) were dissolved in $10 \mathrm{~mL}$ of dry $\mathrm{CHCl}_{3}$ in a $25 \mathrm{~mL}$ two-neck round-bottom flask equipped with a $5 \mathrm{~mL}$ slow-addition apparatus under argon atmosphere at $25^{\circ} \mathrm{C}$. TEA $(0.1 \mathrm{~mL}, 0.73 \mathrm{mmol})$ was added dropwise to the solution and the mixture was stirred for about $24 \mathrm{~h}$. The reaction mixture was quenched with $20 \mathrm{~mL}$ of water and washed with $30 \mathrm{~mL}$ of saturated $\mathrm{NaHCO}_{3}$ (aq.). The combined organic layer was dried with anhydrous $\mathrm{Na}_{2} \mathrm{SO}_{4}$ and the solvent was evaporated to afford the crude product, which was further purified by recycling preparative SEC to give the $\boldsymbol{p}$ - $\mathbf{R}_{\mathbf{1 6}}$ as colorless oil liquid.

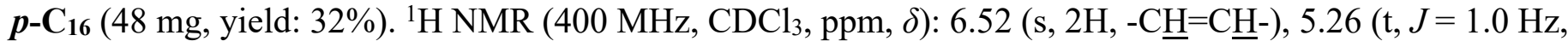
2H, - $\underline{\mathrm{H}}(-\mathrm{O}-) \mathrm{C}-), 3.74\left(\mathrm{dd}, J=9.5,3.1 \mathrm{~Hz}, 15 \mathrm{H},-\mathrm{SC} \underline{\mathrm{H}}(\mathrm{C}=\mathrm{O}) \mathrm{CH}_{2}-\right), 3.55(\mathrm{dt}, J=13.1,7.6 \mathrm{~Hz}, 32 \mathrm{H},-$ $\left.\mathrm{NC}_{2} \mathrm{CH}_{2}-\right), 3.14,3.05\left(\mathrm{~m}, 30 \mathrm{H},-\mathrm{CHC}_{2}(\mathrm{C}=\mathrm{O})-\right), 2.95\left(\mathrm{t}, J=7.1 \mathrm{~Hz}, 2 \mathrm{H}, \mathrm{CH}_{3}(\mathrm{O}=\mathrm{C}) \mathrm{SC} \underline{H}_{2} \mathrm{CH}_{2}-\right), 2.87$ (s, $2 \mathrm{H},-\mathrm{C} \underline{\mathrm{H}}(-\mathrm{C}=\mathrm{O}) \mathrm{C} \underline{H}-), 2.47-2.63\left(\mathrm{~m}, 542 \mathrm{H},-\underline{\mathrm{CH}}_{2} \mathrm{SC}_{\mathrm{H}_{2}}-,-\mathrm{C}_{2} \mathrm{SCH}-\right), 2.35\left(\mathrm{~s}, 3 \mathrm{H}, \mathrm{C}_{3}(\mathrm{O}=\mathrm{C}-) \mathrm{SCH}_{2} \mathrm{CH}_{2}-\right), 1.83$ 
(m, 64H, $\left.-\mathrm{CH}_{2} \mathrm{CH}_{2} \mathrm{CH}_{2} \mathrm{~S}-\right), 1.54\left(\mathrm{~m}, 192 \mathrm{H},-\mathrm{CH}_{2} \mathrm{CH}_{2} \mathrm{CH}_{3}\right), 1.42\left(\mathrm{~m}, 192 \mathrm{H},-\mathrm{CH}_{2} \mathrm{C}_{2} \mathrm{CH}_{3}\right), 1.03$ (m, 256H, $\left.\mathrm{SiC}_{2} \mathrm{CH}_{2}-\right), 0.94\left(\mathrm{~m}, 288 \mathrm{H},-\mathrm{CH}_{2} \mathrm{CH}_{2} \mathrm{C}_{3}\right)$.

\subsection{Discrete Block Polymeric Chains}

The deprotection and coupling reactions follow similar approach as described in section $\mathbf{3 . 2}$.

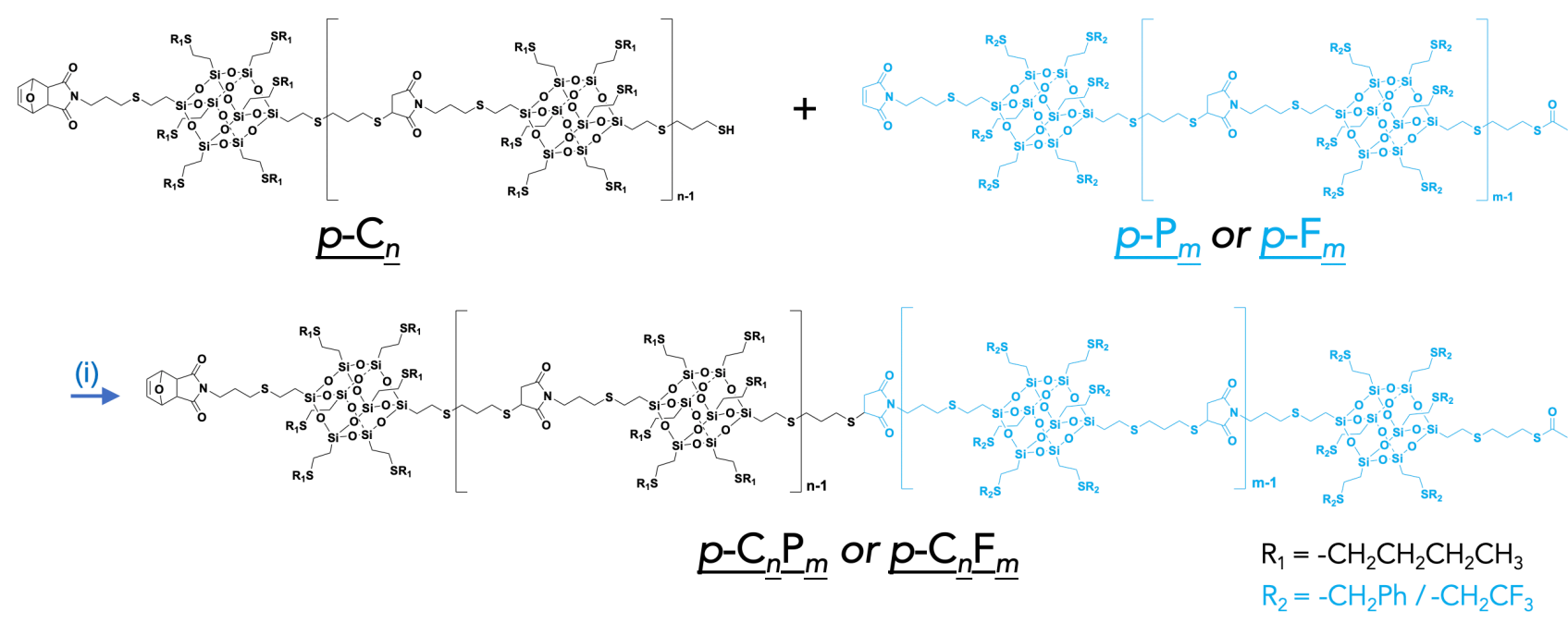

Scheme S3. Synthetic Route of Block Polymeric Chains: (i) TEA, $\mathrm{CHCl}_{3}, 25^{\circ} \mathrm{C}, 8 \mathrm{~h}$.

p- $\mathbf{C}_{1} \mathbf{F}_{1}\left(560 \mathrm{mg}\right.$, yield: 74\%). ${ }^{1} \mathrm{H}$ NMR (400 MHz, $\left.\mathrm{CDCl}_{3}, \mathrm{ppm}, \delta\right): 6.52(\mathrm{~s}, 2 \mathrm{H},-\mathrm{C} \underline{\mathrm{H}}=\mathrm{C} \underline{\mathrm{H}}-)$, $5.26(\mathrm{t}, J=1.0$ $\mathrm{Hz}, 2 \mathrm{H},-\mathrm{C} \underline{\mathrm{H}}(-\mathrm{O}-) \mathrm{C}-), 3.74\left(\mathrm{dd}, J=9.1,3.6 \mathrm{~Hz}, 1 \mathrm{H},-\mathrm{SC} \underline{\mathrm{H}}(\mathrm{C}=\mathrm{O}) \mathrm{CH}_{2}-\right), 3.59$ (dt, $J=13.1,7.6 \mathrm{~Hz}, 4 \mathrm{H},-$ $\left.\mathrm{NC}_{2} \mathrm{CH}_{2}-\right), 3.06-3.19\left(\mathrm{~m}, 14 \mathrm{H},-\mathrm{SC}_{2} \mathrm{CF}_{3},-\mathrm{CHC}_{2}(\mathrm{C}=\mathrm{O})-\right), 2.97\left(\mathrm{t}, J=7.1 \mathrm{~Hz}, 2 \mathrm{H}, \mathrm{CH}_{3}(\mathrm{O}=\mathrm{C}) \mathrm{SC}_{2} \mathrm{CH}_{2}-\right)$, $2.86(\mathrm{~s}, 2 \mathrm{H},-\mathrm{C} \underline{\mathrm{H}}(-\mathrm{C}=\mathrm{O}) \mathrm{C} \underline{\mathrm{H}}-), 2.48-2.77\left(\mathrm{~m}, 54 \mathrm{H},-\mathrm{C}_{2} \mathrm{SCH}_{2} \mathrm{CF}_{3},-\mathrm{C}_{2} \mathrm{SC}_{2}-{ }_{2},-\underline{\mathrm{H}}_{2} \mathrm{SCH}-\right), 2.34(\mathrm{~s}, 3 \mathrm{H}$, $\left.\underline{\mathrm{C}}_{3}(\mathrm{O}=\mathrm{C}-) \mathrm{SCH}_{2} \mathrm{CH}_{2}-\right), 1.84\left(\mathrm{~m}, 8 \mathrm{H},-\mathrm{CH}_{2} \underline{\mathrm{C}}_{2} \mathrm{CH}_{2} \mathrm{~S}-\right), 1.58\left(\mathrm{~m}, 12 \mathrm{H},-\mathrm{CH}_{2} \mathrm{CH}_{2} \mathrm{CH}_{3}\right), 1.41(\mathrm{~m}, 12 \mathrm{H},-$ $\left.\mathrm{CH}_{2} \underline{\mathrm{C}}_{2} \mathrm{CH}_{3}\right), 1.03\left(\mathrm{~m}, 32 \mathrm{H},-\mathrm{SiC}_{2} \mathrm{CH}_{2}-\right), 0.94\left(\mathrm{t}, J=7.3 \mathrm{~Hz}, 18 \mathrm{H},-\mathrm{CH}_{2} \mathrm{CH}_{2} \mathrm{C}_{3}\right)$.

p- $\mathbf{C}_{4} \mathbf{F}_{4}(120 \mathrm{mg}$, yield: $51 \%) .{ }^{1} \mathrm{H}$ NMR $\left(400 \mathrm{MHz}, \mathrm{CDCl}_{3}, \mathrm{ppm}, \delta\right): 6.52(\mathrm{~s}, 2 \mathrm{H},-\mathrm{C} \underline{\mathrm{H}}=\mathrm{C} \underline{\mathrm{H}}-), 5.26(\mathrm{t}, J=1.0$ $\mathrm{Hz}, 2 \mathrm{H},-\mathrm{C} \underline{\mathrm{H}}(-\mathrm{O}-) \mathrm{C}-), 3.75\left(\mathrm{dd}, J=9.1,3.6 \mathrm{~Hz}, 7 \mathrm{H},-\mathrm{SC} \underline{\mathrm{H}}(\mathrm{C}=\mathrm{O}) \mathrm{CH}_{2}-\right), 3.58(\mathrm{dt}, J=13.9,7.0 \mathrm{~Hz}, 16 \mathrm{H},-$ $\left.\mathrm{NC}_{2} \mathrm{CH}_{2}-\right), 2.99-3.20\left(\mathrm{~m}, 62 \mathrm{H},-\mathrm{SCH}_{2} \mathrm{CF}_{3},-\mathrm{CHC}_{2}(\mathrm{C}=\mathrm{O})-\right), 2.92\left(\mathrm{t}, J=7.3 \mathrm{~Hz}, 2 \mathrm{H}, \mathrm{CH}_{3}(\mathrm{O}=\mathrm{C}) \mathrm{SC}_{2} \mathrm{CH}_{2}-\right)$, $2.85(\mathrm{~s}, 2 \mathrm{H},-\mathrm{C} \underline{\mathrm{H}}(-\mathrm{C}=\mathrm{O}) \mathrm{C} \underline{\mathrm{H}}-), 2.51-2.78\left(\mathrm{~m}, 222 \mathrm{H},-\underline{\mathrm{C}}_{2} \mathrm{SCH}_{2} \mathrm{CF}_{3},-\underline{\mathrm{C}}_{2} \mathrm{SC}_{2}-\underline{\mathrm{H}}_{2},-\underline{\mathrm{C}}_{2} \mathrm{SCH}-\right), 2.35$ (s, 3H, 
$\left.\mathrm{C}_{3}(\mathrm{O}=\mathrm{C}-) \mathrm{SCH}_{2} \mathrm{CH}_{2}-\right), 1.86\left(\mathrm{~m}, 32 \mathrm{H},-\mathrm{CH}_{2} \underline{\mathrm{CH}}_{2} \mathrm{CH}_{2} \mathrm{~S}-\right), 1.58\left(\mathrm{~m}, 48 \mathrm{H},-\mathrm{C}_{2} \mathrm{CH}_{2} \mathrm{CH}_{3}\right), 1.43(\mathrm{~m}, 48 \mathrm{H},-$ $\left.\mathrm{CH}_{2} \underline{\mathrm{C}}_{2} \mathrm{CH}_{3}\right), 1.03\left(\mathrm{~m}, 128 \mathrm{H},-\mathrm{SiC}_{2} \mathrm{CH}_{2}-\right), 0.94$ (t, $J=7.3 \mathrm{~Hz}, 72 \mathrm{H},-\mathrm{CH}_{2} \mathrm{CH}_{2} \mathrm{C}_{3}$ ).

p-C $\mathbf{C}_{1}$ (760 mg, yield: 77\%). ${ }^{1} \mathrm{H}$ NMR (400 MHz, $\left.\mathrm{CDCl}_{3}, \mathrm{ppm}, \delta\right):$ 7.25-7.34 (m, 30H, $\left.-\mathrm{SCH}_{2} \underline{\mathrm{Ph}}\right), 6.52(\mathrm{~s}$, $2 \mathrm{H},-\mathrm{C} \underline{\mathrm{H}}=\mathrm{C} \underline{\mathrm{H}}-), 5.27$ (t, $J=1.0 \mathrm{~Hz}, 2 \mathrm{H},-\mathrm{C} \underline{\mathrm{H}}(-\mathrm{O}-) \mathrm{C}-), 3.70-3.74\left(\mathrm{~m}, 13 \mathrm{H},-\mathrm{SC}_{2} \mathrm{Ph},-\mathrm{SC} \underline{\mathrm{H}}(\mathrm{C}=\mathrm{O}) \mathrm{CH}_{2}-\right), 3.59$ $\left(\mathrm{dt}, J=14.1,7.4 \mathrm{~Hz}, 4 \mathrm{H},-\mathrm{NC}_{2} \mathrm{CH}_{2}-\right), 3.14,3.05\left(\mathrm{~m}, 2 \mathrm{H},-\mathrm{CHC}_{2}(\mathrm{C}=\mathrm{O})-\right), 2.95$ (t, $J=7.3 \mathrm{~Hz}, 2 \mathrm{H}$, $\left.\mathrm{CH}_{3}(\mathrm{O}=\mathrm{C}) \mathrm{SC}_{2} \mathrm{CH}_{2}-\right), 2.87(\mathrm{~s}, 2 \mathrm{H},-\mathrm{C} \underline{\mathrm{H}}(-\mathrm{C}=\mathrm{O}) \mathrm{C} \underline{\mathrm{H}}-), 2.47-2.63\left(\mathrm{~m}, 54 \mathrm{H},-\underline{\mathrm{C}}_{2} \mathrm{SC}_{2}{ }^{-},-\underline{\mathrm{C}}_{2} \mathrm{SCH}-\right), 2.35$ (s, $\left.3 \mathrm{H}, \underline{\mathrm{C}}_{3}(\mathrm{O}=\mathrm{C}-) \mathrm{SCH}_{2} \mathrm{CH}_{2}-\right), 1.83\left(\mathrm{~m}, 8 \mathrm{H},-\mathrm{CH}_{2} \underline{\mathrm{C}}_{2} \mathrm{CH}_{2} \mathrm{~S}-\right), 1.55\left(\mathrm{~m}, 12 \mathrm{H},-\underline{\mathrm{C}}_{2} \mathrm{CH}_{2} \mathrm{CH}_{3}\right), 1.44(\mathrm{~m}, 12 \mathrm{H},-$ $\left.\mathrm{CH}_{2} \underline{\mathrm{C}}_{2} \mathrm{CH}_{3}\right), 1.03\left(\mathrm{~m}, 32 \mathrm{H},-\mathrm{SiC}_{2} \mathrm{CH}_{2}-\right), 0.94$ (t, $J=7.3 \mathrm{~Hz}, 18 \mathrm{H},-\mathrm{CH}_{2} \mathrm{CH}_{2} \mathrm{C}_{3}$ ).

p- $\mathbf{C}_{2} \mathbf{P}_{2}$ (378 mg, yield: 54\%). ${ }^{1} \mathrm{H}$ NMR (400 MHz, $\left.\mathrm{CDCl}_{3}, \mathrm{ppm}, \delta\right): 7.25-7.34\left(\mathrm{~m}, 60 \mathrm{H},-\mathrm{SCH}_{2} \underline{\mathrm{Ph}}\right), 6.51(\mathrm{~s}$, $2 \mathrm{H},-\mathrm{C} \underline{\mathrm{H}}=\mathrm{C} \underline{\mathrm{H}}-), 5.26$ (t, $J=1.0 \mathrm{~Hz}, 2 \mathrm{H},-\mathrm{C} \underline{\mathrm{H}}(-\mathrm{O}-) \mathrm{C}-), 3.70-3.74\left(\mathrm{~m}, 27 \mathrm{H},-\mathrm{SC}_{2} \mathrm{Ph},-\mathrm{SC} \underline{\mathrm{H}}(\mathrm{C}=\mathrm{O}) \mathrm{CH}_{2}-\right), 3.59$ $\left(\mathrm{dt}, J=13.1,7.5 \mathrm{~Hz}, 8 \mathrm{H},-\mathrm{NC}_{2} \mathrm{CH}_{2}-\right), 3.14,3.05$ (m, 6H, $\left.-\mathrm{CHC} \underline{H}_{2}(\mathrm{C}=\mathrm{O})-\right), 2.97$ (t, $J=7.1 \mathrm{~Hz}, 2 \mathrm{H}$, $\left.\mathrm{CH}_{3}(\mathrm{O}=\mathrm{C}) \mathrm{SC}_{2} \mathrm{CH}_{2}-\right), 2.85(\mathrm{~s}, 2 \mathrm{H},-\mathrm{C} \underline{\mathrm{H}}(-\mathrm{C}=\mathrm{O}) \mathrm{C} \underline{\mathrm{H}}-), 2.47-2.63\left(\mathrm{~m}, 110 \mathrm{H},-\underline{\mathrm{C}}_{2} \mathrm{SC}_{2} \underline{\mathrm{H}}_{2}-,-\mathrm{C}_{2} \mathrm{SCH}-\right), 2.34$ (s, $\left.3 \mathrm{H}, \underline{\mathrm{C}}_{3}(\mathrm{O}=\mathrm{C}-) \mathrm{SCH}_{2} \mathrm{CH}_{2}-\right), 1.86\left(\mathrm{~m}, 16 \mathrm{H},-\mathrm{CH}_{2} \mathrm{CH}_{2} \mathrm{CH}_{2} \mathrm{~S}-\right), 1.56\left(\mathrm{~m}, 24 \mathrm{H},-\mathrm{CH}_{2} \mathrm{CH}_{2} \mathrm{CH}_{3}\right), 1.42(\mathrm{~m}, 24 \mathrm{H},-$ $\left.\mathrm{CH}_{2} \underline{\mathrm{C}}_{2} \mathrm{CH}_{3}\right), 1.02\left(\mathrm{~m}, 64 \mathrm{H},-\mathrm{SiC}_{2} \mathrm{CH}_{2}-\right), 0.93\left(\mathrm{t}, J=7.7 \mathrm{~Hz}, 36 \mathrm{H},-\mathrm{CH}_{2} \mathrm{CH}_{2} \mathrm{C}_{3}\right)$.

p-C $\mathbf{C}_{4} \mathbf{P}_{4}\left(161 \mathrm{mg}\right.$, yield: 46\%). ${ }^{1} \mathrm{H}$ NMR (400 MHz, $\left.\mathrm{CDCl}_{3}, \mathrm{ppm}, \delta\right): 7.23-7.34\left(\mathrm{~m}, 120 \mathrm{H},-\mathrm{SCH}_{2} \underline{\mathrm{Ph}}\right), 6.52(\mathrm{~s}$, $2 \mathrm{H},-\mathrm{C} \underline{\mathrm{H}}=\mathrm{C} \underline{\mathrm{H}}-), 5.23$ (t, $J=1.0 \mathrm{~Hz}, 2 \mathrm{H},-\mathrm{C} \underline{\mathrm{H}}(-\mathrm{O}-) \mathrm{C}-), 3.70-3.74\left(\mathrm{~m}, 55 \mathrm{H},-\mathrm{SC}_{2} \mathrm{Ph},-\mathrm{SC} \underline{\mathrm{H}}(\mathrm{C}=\mathrm{O}) \mathrm{CH}_{2}-\right), 3.60$ (dt, $\left.J=13.1,7.5 \mathrm{~Hz}, 16 \mathrm{H},-\mathrm{NC}_{2} \mathrm{CH}_{2}-\right), 3.14,3.05$ (m, 14H, - $\left.\mathrm{CHC}_{2}(\mathrm{C}=\mathrm{O})-\right), 2.99$ (t, $J=7.9 \mathrm{~Hz}, 2 \mathrm{H}$, $\left.\mathrm{CH}_{3}(\mathrm{O}=\mathrm{C}) \mathrm{SC}_{2} \mathrm{CH}_{2}-\right), 2.84(\mathrm{~s}, 2 \mathrm{H},-\mathrm{C} \underline{\mathrm{H}}(-\mathrm{C}=\mathrm{O}) \mathrm{C} \underline{H}-), 2.43-2.63\left(\mathrm{~m}, 222 \mathrm{H},-\underline{\mathrm{C}}_{2} \mathrm{SC}_{2} \underline{\mathrm{H}}_{2}-,-\underline{\mathrm{C}}_{2} \mathrm{SCH}-\right), 2.32$ (s, $\left.3 \mathrm{H}, \underline{\mathrm{C}}_{3}(\mathrm{O}=\mathrm{C}-) \mathrm{SCH}_{2} \mathrm{CH}_{2}-\right), 1.85\left(\mathrm{~m}, 32 \mathrm{H},-\mathrm{CH}_{2} \mathrm{C}_{2} \mathrm{CH}_{2} \mathrm{~S}-\right), 1.54\left(\mathrm{~m}, 48 \mathrm{H},-\mathrm{C}_{2} \mathrm{CH}_{2} \mathrm{CH}_{3}\right), 1.42(\mathrm{~m}, 48 \mathrm{H},-$ $\mathrm{CH}_{2} \underline{\mathrm{C}}_{2} \mathrm{CH}_{3}$ ), 1.05 (m, 128H, - $\left.\mathrm{SiC}_{2} \mathrm{CH}_{2}-\right), 0.91$ (t, $J=7.1 \mathrm{~Hz}, 72 \mathrm{H},-\mathrm{CH}_{2} \mathrm{CH}_{2} \mathrm{C}_{3}$ ).

p-C8P8 (37 mg, yield: 34\%). ${ }^{1} \mathrm{H}$ NMR (400 MHz, $\left.\mathrm{CDCl}_{3}, \mathrm{ppm}, \delta\right): 7.25-7.35$ (m, 240H, $\left.-\mathrm{SCH}_{2} \underline{\mathrm{Ph}}\right), 6.51(\mathrm{~s}$, $2 \mathrm{H},-\mathrm{C} \underline{\mathrm{H}}=\mathrm{C} \underline{\mathrm{H}}-), 5.22$ (t, $J=1.0 \mathrm{~Hz}, 2 \mathrm{H},-\mathrm{C} \underline{\mathrm{H}}(-\mathrm{O}-) \mathrm{C}-), 3.68-3.74\left(\mathrm{~s}, 111 \mathrm{H},-\mathrm{SC}_{2} \mathrm{Ph},-\mathrm{SC} \underline{\mathrm{H}}(\mathrm{C}=\mathrm{O}) \mathrm{CH}_{2}-\right), 3.58$ (dt, $\left.J=14.1,7.1 \mathrm{~Hz}, 32 \mathrm{H},-\mathrm{NC}_{2} \mathrm{CH}_{2}-\right), 3.14,3.05$ (m, 30H, - $\left.\mathrm{CHC}_{2}(\mathrm{C}=\mathrm{O})-\right), 2.98$ (t, $J=6.9 \mathrm{~Hz}, 2 \mathrm{H}$, 


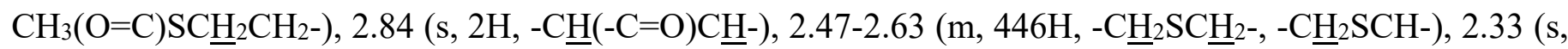
$\left.3 \mathrm{H}, \underline{\mathrm{C}}_{3}(\mathrm{O}=\mathrm{C}-) \mathrm{SCH}_{2} \mathrm{CH}_{2}-\right), 1.87\left(\mathrm{~m}, 64 \mathrm{H},-\mathrm{CH}_{2} \mathrm{C}_{2} \mathrm{CH}_{2} \mathrm{~S}-\right), 1.55\left(\mathrm{~m}, 96 \mathrm{H},-\mathrm{C}_{2} \mathrm{CH}_{2} \mathrm{CH}_{3}\right), 1.43(\mathrm{~m}, 96 \mathrm{H},-$ $\left.\mathrm{CH}_{2} \underline{\mathrm{C}}_{2} \mathrm{CH}_{3}\right), 1.03\left(\mathrm{~m}, 256 \mathrm{H},-\mathrm{SiC}_{2} \mathrm{CH}_{2}-\right), 0.94$ (t, $\left.J=7.6 \mathrm{~Hz}, 144 \mathrm{H},-\mathrm{CH}_{2} \mathrm{CH}_{2} \mathrm{C}_{3}\right)$.

\subsection{Discrete Amphiphilic Block Polymeric Chains $p-C_{n} D$}

\subsubsection{V POSS-SH}

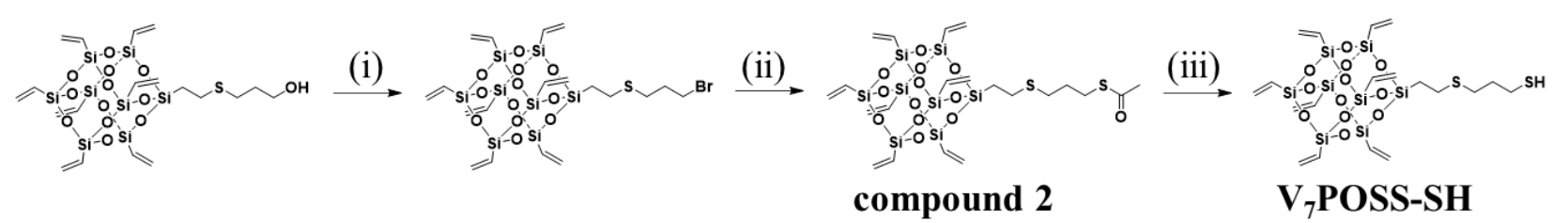

Scheme S4. Synthesis of VPOSS-SH: (i) $\mathrm{CBr}_{4}, \mathrm{PPh}_{3}, \mathrm{CH}_{2} \mathrm{Cl}_{2}, 0{ }^{\circ} \mathrm{C}, 2 \mathrm{~h}$; (ii) potassium thioacetate, $\mathrm{KI}, \mathrm{MeOH} /$ $\mathrm{CH}_{2} \mathrm{Cl}_{2}, 75^{\circ} \mathrm{C}, 12 \mathrm{~h}$; (iii) acetyl chloride, $\mathrm{MeOH} / \mathrm{CH}_{2} \mathrm{Cl}_{2},-78{ }^{\circ} \mathrm{C}, 7 \mathrm{~h}$.

V7POSS-OH (3.63g, $5.0 \mathrm{mmol})$ was dissolved in anhydrous $\mathrm{CH}_{2} \mathrm{Cl}_{2}(50 \mathrm{~mL})$ in a $100 \mathrm{~mL}$ round-bottom flask.

$\mathrm{CBr}_{4}(3.36 \mathrm{~g}, 10.0 \mathrm{mmol})$ was added to the mixture, and the mixture was transferred into an ice bath. When completely dissolved, $\mathrm{PPh}_{3}(2.62 \mathrm{~g}, 10.0 \mathrm{mmol})$ was slowly added. After string for 4 hours at room temperature, $20 \mathrm{~mL}$ of $\mathrm{MeOH}, \mathrm{KI}(20 \mathrm{mg})$, and potassium thioacetate $(0.23 \mathrm{~g}, 2.0 \mathrm{mmol})$ were added, and the mixture was further stirred at $75^{\circ} \mathrm{C}$ for $12 \mathrm{~h}$ under argon atmosphere. The solvent was then removed under vacuum and the crude product was purified by silica gel column chromatography with PE/DCM (10/1, v/v) as eluent to afford compound 2 as a white powder (3.56 g, yield: $91 \%)$.

Compound $2(3.00 \mathrm{~g}, 3.8 \mathrm{mmol})$ was dissolved in $20 \mathrm{~mL}$ of $\mathrm{MeOH}$ and $60 \mathrm{~mL}$ of $\mathrm{CH}_{2} \mathrm{Cl}_{2}$ in a $250 \mathrm{~mL}$ twoneck round-bottom flask. The mixture was cooled to $-78^{\circ} \mathrm{C}$. Acetyl chloride $(15.0 \mathrm{~mL}, 111 \mathrm{mmol})$ was then added dropwise to the solution. After further stirring for $14 \mathrm{~h}$ at room temperature, the reaction mixture was quenched with $20 \mathrm{~mL}$ of water and washed with $30 \mathrm{~mL}$ of saturated $\mathrm{NaHCO}_{3}$ (aq.). The organic layers were combined and washed with $50 \mathrm{~mL}$ of water and dried with anhydrous $\mathrm{Na}_{2} \mathrm{SO}_{4}$. DCM was removed under 
vacuum to afford $\mathbf{V}_{7}$ POSS-SH as a white powder (2.5 g, yield: $85 \%$ ).

3.4.2 $p-\mathbf{C}_{n} \mathbf{V}$ (scheme S3). We prepared a library of homopolymeric chains $\left(p-\mathrm{C}_{n}, n=1,2,3,4,5,6,8,16\right)$ in addition to those have been described in Section 3.2. The deprotection of the maleimide group ( $\boldsymbol{p}$-Mal- $\mathbf{C}_{\mathbf{1}}$, $p$-Mal-C $2, p$-Mal-C $3, p$-Mal-C4, $p$-Mal-C5, $p$-Mal-C6, $p$-Mal-C8) follows similar approach described above.

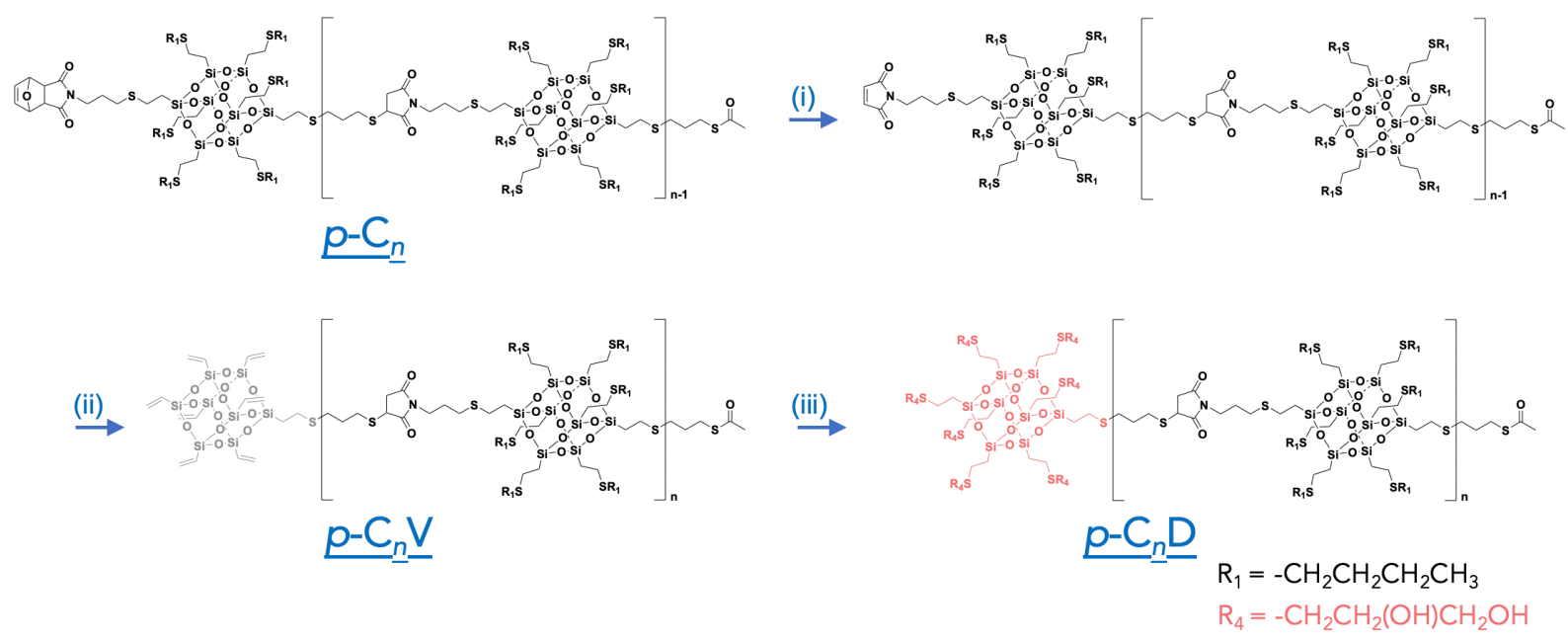

Scheme S5. Synthetic Route of Amphiphilic Block Polymeric Chains $p-\mathrm{C}_{n} \mathrm{D}$ : (i) Toluene, $110{ }^{\circ} \mathrm{C}, 5 \mathrm{~h}$; (ii) $\mathrm{V}_{7} \mathrm{POSS}-\mathrm{SH}, \mathrm{TEA}, \mathrm{CHCl}_{3}, 25^{\circ} \mathrm{C}, 8 \mathrm{~h}$; (iii) 1-thioglycerol, DMPA, THF, $25^{\circ} \mathrm{C}, 365 \mathrm{~nm} \times 30 \mathrm{~min}$.

p-Mal-C $\mathbf{C}_{1}(200 \mathrm{mg}, n=1,2,3,4,5,6,8)$ and V7POSS-SH (3 eq.) were dissolved in $10 \mathrm{~mL}$ of anhydrous $\mathrm{CHCl}_{3}$ in a $25 \mathrm{~mL}$ two-neck round-bottom flask, TEA $(0.1 \mathrm{~mL}, 0.73 \mathrm{mmol})$ was added dropwise to the solution and the mixture was stirred for $24 \mathrm{~h}$. The reaction mixture was quenched with $15 \mathrm{~mL}$ of water and washed with $10 \mathrm{~mL}$ of saturated $\mathrm{NaHCO}_{3}$ (aq.). The combined organic layer was dried with anhydrous $\mathrm{Na}_{2} \mathrm{SO}_{4}$ and the solvent was evaporated to afford the crude product which was purified by recycling preparative SEC to give the $\boldsymbol{p}-\mathbf{C}_{\boldsymbol{n}} \mathbf{V}$ as colorless oil liquid (170 mg 180 mg, yield: $77 \% \sim 82 \%$ ).

p-C1V (180 mg, yield: 82\%). ${ }^{1} \mathrm{H}$ NMR (400 MHz, $\left.\mathrm{CDCl}_{3}, \mathrm{ppm}, \delta\right): 5.89-6.14$ (m, 21H, $\left.\underline{\mathrm{C}}_{2}=\mathrm{C} \underline{\mathrm{H}}-\right)$, 3.73 (dd, $\left.J=9.0,3.6 \mathrm{~Hz}, 1 \mathrm{H},-\mathrm{SC} \underline{\mathrm{H}}(\mathrm{C}=\mathrm{O}) \mathrm{CH}_{2^{-}}\right), 3.63\left(\mathrm{t}, J=7.2 \mathrm{~Hz}, 2 \mathrm{H},-\mathrm{NC}_{2} \mathrm{CH}_{2^{-}}\right), 3.14,3.05(\mathrm{~m}, 2 \mathrm{H},-$ $\left.\mathrm{CHC}_{2}(\mathrm{C}=\mathrm{O})-\right), 2.98\left(\mathrm{t}, J=7.2 \mathrm{~Hz}, 2 \mathrm{H}, \mathrm{CH}_{3}(\mathrm{O}=\mathrm{C}) \mathrm{SCH}_{2} \mathrm{CH}_{2}-\right), 2.51-2.65\left(\mathrm{~m}, 38 \mathrm{H},-\underline{\mathrm{C}}_{2} \mathrm{SC}_{2}-\underline{H}_{2},-\underline{\mathrm{H}}_{2} \mathrm{SCH}-\right)$, $2.35\left(\mathrm{~s}, 3 \mathrm{H}, \mathrm{CH}_{3}(\mathrm{O}=\mathrm{C}-) \mathrm{SCH}_{2} \mathrm{CH}_{2}-\right), 1.86\left(\mathrm{~m}, 6 \mathrm{H},-\mathrm{CH}_{2} \underline{\mathrm{C}}_{2} \mathrm{CH}_{2} \mathrm{~S}-\right), 1.57\left(\mathrm{~m}, 12 \mathrm{H},-\underline{\mathrm{H}}_{2} \mathrm{CH}_{2} \mathrm{CH}_{3}\right), 1.43(\mathrm{~m}$, 
$\left.12 \mathrm{H},-\mathrm{CH}_{2} \underline{\mathrm{CH}}_{2} \mathrm{CH}_{3}\right), 1.03$ (m, 18H, $\left.-\mathrm{SiC}_{2} \mathrm{CH}_{2}-\right), 0.93$ (t, $J=7.2 \mathrm{~Hz}, 18 \mathrm{H},-\mathrm{CH}_{2} \mathrm{CH}_{2} \mathrm{C}_{3}$ ).

p- $\mathbf{C}_{2} \mathbf{V}\left(178 \mathrm{mg}\right.$, yield: 81\%). ${ }^{1} \mathrm{H}$ NMR (400 MHz, $\left.\mathrm{CDCl}_{3}, \mathrm{ppm}, \delta\right): 5.85-6.15$ (m, 21H, $\left.\underline{\mathrm{C}}_{2}=\mathrm{C} \underline{\mathrm{H}}-\right)$, 3.74 (dd, $\left.J=9.3,3.6 \mathrm{~Hz}, 2 \mathrm{H},-\mathrm{SC} \underline{\mathrm{H}}(\mathrm{C}=\mathrm{O}) \mathrm{CH}_{2}-\right), 3.64\left(\mathrm{t}, J=7.2 \mathrm{~Hz}, 4 \mathrm{H},-\mathrm{NC}_{2} \mathrm{CH}_{2}\right), 3.14,3.05(\mathrm{~m}, 4 \mathrm{H},-$ $\left.\mathrm{CHC}_{2}(\mathrm{C}=\mathrm{O})-\right), 2.96\left(\mathrm{t}, J=7.2 \mathrm{~Hz}, 2 \mathrm{H}, \mathrm{CH}_{3}(\mathrm{O}=\mathrm{C}) \mathrm{SCH}_{2} \mathrm{CH}_{2}-\right), 2.51-2.65\left(\mathrm{~m}, 72 \mathrm{H},-\underline{\mathrm{C}}_{2} \mathrm{SC}_{2}-{ }^{-},-\underline{\mathrm{H}}_{2} \mathrm{SCH}-\right)$, $2.34\left(\mathrm{~s}, 3 \mathrm{H}, \mathrm{C}_{3}(\mathrm{O}=\mathrm{C}-) \mathrm{SCH}_{2} \mathrm{CH}_{2}-\right), 1.85\left(\mathrm{~m}, 10 \mathrm{H},-\mathrm{CH}_{2} \mathrm{C}_{2} \mathrm{CH}_{2} \mathrm{~S}-\right), 1.53\left(\mathrm{~m}, 24 \mathrm{H},-\underline{\mathrm{H}}_{2} \mathrm{CH}_{2} \mathrm{CH}_{3}\right), 1.45(\mathrm{~m}$, 24H, $\left.-\mathrm{CH}_{2} \underline{\mathrm{CH}}_{2} \mathrm{CH}_{3}\right), 1.04$ (m, 34H, $\left.-\mathrm{SiC}_{2} \mathrm{CH}_{2}-\right), 0.96$ (t, $\left.J=7.9 \mathrm{~Hz}, 36 \mathrm{H},-\mathrm{CH}_{2} \mathrm{CH}_{2} \mathrm{C}_{3}\right)$.

p-C ${ }_{3} \mathbf{V}$ (176 mg, yield: 81\%). ${ }^{1} \mathrm{H}$ NMR (400 MHz, $\left.\mathrm{CDCl}_{3}, \mathrm{ppm}, \delta\right): 5.83-6.13$ (m, 21H, $\left.\underline{\mathrm{C}}_{2}=\mathrm{C} \underline{\mathrm{H}}-\right)$ ), 3.72 (dd, $\left.J=9.6,3.2 \mathrm{~Hz}, 3 \mathrm{H},-\mathrm{SC} \underline{\mathrm{H}}(\mathrm{C}=\mathrm{O}) \mathrm{CH}_{2}-\right), 3.67\left(\mathrm{t}, J=7.4 \mathrm{~Hz}, 6 \mathrm{H},-\mathrm{NC}_{2} \mathrm{CH}_{2}\right), 3.14,3.05(\mathrm{~m}, 6 \mathrm{H},-$ $\left.\mathrm{CHC}_{2}(\mathrm{C}=\mathrm{O})-\right), 2.92\left(\mathrm{t}, J=7.2 \mathrm{~Hz}, 2 \mathrm{H}, \mathrm{CH}_{3}(\mathrm{O}=\mathrm{C}) \mathrm{SC}_{2}{ }_{2} \mathrm{CH}_{2}-\right), 2.51-2.65\left(\mathrm{~m}, 106 \mathrm{H},-\underline{\mathrm{C}}_{2} \mathrm{SC}_{2}-\underline{\mathrm{H}}_{2},-\underline{\mathrm{HCH}}_{2}\right)$, $2.36\left(\mathrm{~s}, 3 \mathrm{H}, \mathrm{C}_{3}(\mathrm{O}=\mathrm{C}-) \mathrm{SCH}_{2} \mathrm{CH}_{2}-\right), 1.84\left(\mathrm{~m}, 14 \mathrm{H},-\mathrm{CH}_{2} \mathrm{CH}_{2} \mathrm{CH}_{2} \mathrm{~S}-\right), 1.54\left(\mathrm{~m}, 36 \mathrm{H},-\underline{\mathrm{C}}_{2} \mathrm{CH}_{2} \mathrm{CH}_{3}\right), 1.47(\mathrm{~m}$, $\left.36 \mathrm{H},-\mathrm{CH}_{2} \underline{\mathrm{CH}}_{2} \mathrm{CH}_{3}\right), 1.03\left(\mathrm{~m}, 50 \mathrm{H},-\mathrm{SiC}_{2} \mathrm{CH}_{2}-\right), 0.94$ (t, $\left.J=7.9 \mathrm{~Hz}, 54 \mathrm{H},-\mathrm{CH}_{2} \mathrm{CH}_{2} \mathrm{C}_{3}\right)$.

p- $\mathbf{C}_{4} \mathrm{~V}$ (175 mg, yield: 80\%). ${ }^{1} \mathrm{H}$ NMR (400 MHz, $\left.\mathrm{CDCl}_{3}, \mathrm{ppm}, \delta\right): 5.83-6.15$ (m, 21H, $\left.\underline{\mathrm{C}}_{2}=\mathrm{C} \underline{\mathrm{H}}-\right)$, 3.71 (dd, $\left.J=9.1,3.8 \mathrm{~Hz}, 4 \mathrm{H},-\mathrm{SC} \underline{\mathrm{H}}(\mathrm{C}=\mathrm{O}) \mathrm{CH}_{2}-\right), 3.62\left(\mathrm{t}, J=7.1 \mathrm{~Hz}, 8 \mathrm{H},-\mathrm{NC}_{2} \mathrm{CH}_{2}\right), 3.14,3.05(\mathrm{~m}, 8 \mathrm{H},-$ $\left.\mathrm{CHC}_{2}(\mathrm{C}=\mathrm{O})-\right), 2.99\left(\mathrm{t}, J=7.2 \mathrm{~Hz}, 2 \mathrm{H}, \mathrm{CH}_{3}(\mathrm{O}=\mathrm{C}) \mathrm{SC}_{2} \mathrm{CH}_{2}-\right), 2.51-2.65\left(\mathrm{~m}, 140 \mathrm{H},-\underline{\mathrm{C}}_{2} \mathrm{SC}_{2}-,-\underline{\mathrm{H}}_{2} \mathrm{SCH}-\right)$, $2.32\left(\mathrm{~s}, 3 \mathrm{H}, \mathrm{CH}_{3}(\mathrm{O}=\mathrm{C}-) \mathrm{SCH}_{2} \mathrm{CH}_{2}-\right), 1.83\left(\mathrm{~m}, 18 \mathrm{H},-\mathrm{CH}_{2} \mathrm{CH}_{2} \mathrm{CH}_{2} \mathrm{~S}-\right), 1.52\left(\mathrm{~m}, 48 \mathrm{H},-\underline{\mathrm{C}}_{2} \mathrm{CH}_{2} \mathrm{CH}_{3}\right), 1.44$ (m, $\left.48 \mathrm{H},-\mathrm{CH}_{2} \underline{\mathrm{CH}}_{2} \mathrm{CH}_{3}\right), 1.03\left(\mathrm{~m}, 66 \mathrm{H},-\mathrm{SiC}_{2} \mathrm{CH}_{2}-\right), 0.92\left(\mathrm{t}, J=7.2 \mathrm{~Hz}, 72 \mathrm{H},-\mathrm{CH}_{2} \mathrm{CH}_{2} \mathrm{C}_{3}\right)$.

p-C5V (172 mg, yield: 78\%). ${ }^{1} \mathrm{H}$ NMR (400 MHz, $\left.\mathrm{CDCl}_{3}, \mathrm{ppm}, \delta\right): 5.81-6.14$ (m, 21H, $\left.\underline{\mathrm{C}}_{2}=\mathrm{C} \underline{\mathrm{H}}-\right), 3.70$ (dd, $\left.J=9.5,3.8 \mathrm{~Hz}, 5 \mathrm{H},-\mathrm{SC} \underline{\mathrm{H}}(\mathrm{C}=\mathrm{O}) \mathrm{CH}_{2}-\right), 3.62\left(\mathrm{t}, J=7.3 \mathrm{~Hz}, 10 \mathrm{H},-\mathrm{NC}_{2} \mathrm{CH}_{2}-\right), 3.14,3.05(\mathrm{~m}, 10 \mathrm{H},-$ $\left.\mathrm{CHC}_{2}(\mathrm{C}=\mathrm{O})-\right), 2.96\left(\mathrm{t}, J=7.3 \mathrm{~Hz}, 2 \mathrm{H}, \mathrm{CH}_{3}(\mathrm{O}=\mathrm{C}) \mathrm{SC}_{2} \mathrm{CH}_{2}-\right), 2.51-2.65$ (m, 174H, $\left.-\underline{\mathrm{C}}_{2} \mathrm{SC}_{2}-\underline{\mathrm{H}}_{2},-\underline{\mathrm{H}}_{2} \mathrm{SCH}-\right)$, $2.31\left(\mathrm{~s}, 3 \mathrm{H}, \mathrm{C}_{3}(\mathrm{O}=\mathrm{C}-) \mathrm{SCH}_{2} \mathrm{CH}_{2}-\right), 1.81\left(\mathrm{~m}, 22 \mathrm{H},-\mathrm{CH}_{2} \mathrm{CH}_{2} \mathrm{CH}_{2} \mathrm{~S}-\right), 1.52\left(\mathrm{~m}, 60 \mathrm{H},-\underline{\mathrm{C}}_{2} \mathrm{CH}_{2} \mathrm{CH}_{3}\right), 1.43(\mathrm{~m}$, $\left.60 \mathrm{H},-\mathrm{CH}_{2} \underline{\mathrm{C}}_{2} \mathrm{CH}_{3}\right), 1.06$ (m, 82H, $\left.-\mathrm{SiC}_{2} \mathrm{CH}_{2}-\right), 0.91$ (t, $\left.J=7.9 \mathrm{~Hz}, 90 \mathrm{H},-\mathrm{CH}_{2} \mathrm{CH}_{2} \mathrm{C}_{3}\right)$.

p-C6V (170 mg, yield: 77\%). ${ }^{1} \mathrm{H}$ NMR (400 MHz, $\left.\mathrm{CDCl}_{3}, \mathrm{ppm}, \delta\right): 5.87-6.18$ (m, 21H, $\left.\mathrm{C}_{2}=\mathrm{C} \underline{\mathrm{H}}-\right)$, 3.73 (dd, $\left.J=9.5,3.8 \mathrm{~Hz}, 6 \mathrm{H},-\mathrm{SC} \underline{\mathrm{H}}(\mathrm{C}=\mathrm{O}) \mathrm{CH}_{2}-\right), 3.62\left(\mathrm{t}, J=7.3 \mathrm{~Hz}, 12 \mathrm{H},-\mathrm{NC}_{2} \mathrm{CH}_{2}-\right), 3.14,3.05(\mathrm{~m}, 12 \mathrm{H},-$ 
$\left.\mathrm{CHC}_{2}(\mathrm{C}=\mathrm{O})-\right), 2.92$ (t, $\left.J=7.3 \mathrm{~Hz}, 2 \mathrm{H}, \mathrm{CH}_{3}(\mathrm{O}=\mathrm{C}) \mathrm{SCH}_{2} \mathrm{CH}_{2}-\right), 2.51-2.65$ (m, 208H, - $\underline{\mathrm{H}}_{2} \mathrm{SC}_{2}-$, - $\left.\underline{\mathrm{H}}_{2} \mathrm{SCH}-\right)$, $2.39\left(\mathrm{~s}, 3 \mathrm{H}, \mathrm{C}_{3}(\mathrm{O}=\mathrm{C}-) \mathrm{SCH}_{2} \mathrm{CH}_{2}-\right), 1.82\left(\mathrm{~m}, 26 \mathrm{H},-\mathrm{CH}_{2} \underline{\mathrm{C}}_{2} \mathrm{CH}_{2} \mathrm{~S}-\right), 1.537\left(\mathrm{~m}, 72 \mathrm{H},-\underline{\mathrm{C}}_{2} \mathrm{CH}_{2} \mathrm{CH}_{3}\right), 1.41(\mathrm{~m}$, $\left.72 \mathrm{H},-\mathrm{CH}_{2} \underline{\mathrm{C}}_{2} \mathrm{CH}_{3}\right), 1.06\left(\mathrm{~m}, 98 \mathrm{H},-\mathrm{SiCH}_{2} \mathrm{CH}_{2}-\right), 0.91\left(\mathrm{t}, J=7.9 \mathrm{~Hz}, 108 \mathrm{H},-\mathrm{CH}_{2} \mathrm{CH}_{2} \mathrm{C}_{3}\right)$.

p-C8V (170 mg, yield: 77\%). ${ }^{1} \mathrm{H}$ NMR (400 MHz, $\left.\mathrm{CDCl}_{3}, \mathrm{ppm}, \delta\right): 5.81-6.11$ (m, 21H, $\left.\underline{\mathrm{C}}_{2}=\mathrm{C} \underline{\mathrm{H}}-\right)$, $3.72(\mathrm{dd}$, $\left.J=9.5,3.8 \mathrm{~Hz}, 8 \mathrm{H},-\mathrm{SC} \underline{\mathrm{H}}(\mathrm{C}=\mathrm{O}) \mathrm{CH}_{2}-\right), 3.62\left(\mathrm{t}, J=7.3 \mathrm{~Hz}, 16 \mathrm{H},-\mathrm{NC}_{2} \mathrm{CH}_{2}-\right), 3.14,3.05(\mathrm{~m}, 16 \mathrm{H},-$ $\left.\mathrm{CHC}_{2}(\mathrm{C}=\mathrm{O})-\right), 2.92$ (t, $\left.J=7.3 \mathrm{~Hz}, 2 \mathrm{H}, \mathrm{CH}_{3}(\mathrm{O}=\mathrm{C}) \mathrm{SC}_{2} \mathrm{CH}_{2}-\right), 2.51-2.65$ (m, 276H, - $\left.\underline{\mathrm{H}}_{2} \mathrm{SC}_{2}-{ }_{2},-\underline{\mathrm{C}}_{2} \mathrm{SCH}-\right)$, $2.33\left(\mathrm{~s}, 3 \mathrm{H}, \mathrm{C}_{3}(\mathrm{O}=\mathrm{C}-) \mathrm{SCH}_{2} \mathrm{CH}_{2}-\right), 1.86\left(\mathrm{~m}, 34 \mathrm{H},-\mathrm{CH}_{2} \mathrm{CH}_{2} \mathrm{CH}_{2} \mathrm{~S}-\right), 1.54\left(\mathrm{~m}, 96 \mathrm{H},-\underline{\mathrm{C}}_{2} \mathrm{CH}_{2} \mathrm{CH}_{3}\right), 1.43(\mathrm{~m}$, 96H, $-\mathrm{CH}_{2} \underline{\mathrm{C}}_{2} \mathrm{CH}_{3}$ ), 1.04 (m, 130H, $\left.-\mathrm{SiC}_{2} \mathrm{CH}_{2}-\right), 0.93$ (t, $J=7.9 \mathrm{~Hz}, 144 \mathrm{H},-\mathrm{CH}_{2} \mathrm{CH}_{2} \mathrm{CH}_{3}$ ).

3.4.3 $p-\mathbf{C}_{n} D$. A typical procedure is described as follows: $\boldsymbol{p}-\mathbf{C}_{n} \mathbf{V}, 1$-thioglycerol (2 eq. to vinyl group), and DMPA (0.03 eq.) were dissolved in $4 \mathrm{~mL}$ of THF. The solution was irradiated under $365 \mathrm{~nm}$ UV light in a UV reactor for $35 \mathrm{~min}$. The solution is concentrated and purified by recycling preparative SEC to give the $\boldsymbol{p}-\mathbf{C}_{\boldsymbol{n}} \mathbf{D}$ as colorless oil liquid with $70 \%-85 \%$ yield.

p-C1D (153 mg, yield: 82\%). ${ }^{1} \mathrm{H}$ NMR (400 MHz, $\left.\mathrm{CDCl}_{3}, \mathrm{ppm}, \delta\right): 3.71-3.82(\mathrm{~m}, 22 \mathrm{H},-\mathrm{C} \underline{\mathrm{H}}(\mathrm{OH}) \mathrm{C} \underline{\mathrm{H}} 2 \mathrm{OH}$, $\left.\mathrm{SC} \underline{\mathrm{H}}(\mathrm{C}=\mathrm{O}) \mathrm{CH}_{2}-\right), 3.57\left(\mathrm{t}, J=7.0 \mathrm{~Hz}, 2 \mathrm{H},-\mathrm{NC}_{2} \mathrm{CH}_{2}-\right), 3.14,3.05\left(\mathrm{~m}, 2 \mathrm{H},-\mathrm{CHCH}_{2}(\mathrm{C}=\mathrm{O})-\right), 2.96(\mathrm{t}, J=7.9$ $\left.\mathrm{Hz}, \quad 2 \mathrm{H}, \quad \mathrm{CH}_{3}(\mathrm{O}=\mathrm{C}) \mathrm{SC}_{2} \mathrm{CH}_{2}-\right), \quad 2.51-2.72 \quad\left(\mathrm{~m}, \quad 66 \mathrm{H}, \quad-\underline{\mathrm{C}}_{2} \mathrm{SC}_{2}-2, \quad-\underline{\mathrm{C}}_{2} \mathrm{SCH}-\right), \quad 2.35 \quad(\mathrm{~s}, \quad 3 \mathrm{H}$, $\left.\mathrm{C}_{3}(\mathrm{O}=\mathrm{C}-) \mathrm{SCH}_{2} \mathrm{CH}_{2}-\right), 1.86\left(\mathrm{~m}, 6 \mathrm{H},-\mathrm{CH}_{2} \underline{\mathrm{CH}}_{2} \mathrm{CH}_{2} \mathrm{~S}-\right), 1.57\left(\mathrm{~m}, 12 \mathrm{H},-\underline{\mathrm{C}}_{2} \mathrm{CH}_{2} \mathrm{CH}_{3}\right), 1.43(\mathrm{~m}, 12 \mathrm{H},-$ $\left.\mathrm{CH}_{2} \underline{\mathrm{C}}_{2} \mathrm{CH}_{3}\right), 1.04\left(\mathrm{~m}, 32 \mathrm{H},-\mathrm{SiC}_{2} \mathrm{CH}_{2}-\right), 0.93$ (t, $\left.J=7.1 \mathrm{~Hz}, 18 \mathrm{H},-\mathrm{CH}_{2} \mathrm{CH}_{2} \mathrm{C}_{3}\right)$.

p-C $\mathbf{C}_{2} \mathbf{D}$ (142 mg, yield: 80\%). ${ }^{1} \mathrm{H}-\mathrm{NMR}\left(400 \mathrm{MHz}, \mathrm{CDCl}_{3}, \mathrm{ppm}, \delta\right): 3.70-3.82\left(\mathrm{~m}, 23 \mathrm{H},-\mathrm{C} \underline{\mathrm{H}}(\mathrm{OH}) \mathrm{C}_{2} \mathrm{OH},-\right.$ $\left.\mathrm{SC} \underline{\mathrm{H}}(\mathrm{C}=\mathrm{O}) \mathrm{CH}_{2}-\right), 3.58\left(\mathrm{t}, J=7.0 \mathrm{~Hz}, 4 \mathrm{H},-\mathrm{NC}_{2} \mathrm{CH}_{2}-\right), 3.13,3.05\left(\mathrm{~m}, 4 \mathrm{H},-\mathrm{CHCH}_{2}(\mathrm{C}=\mathrm{O})-\right), 2.98(\mathrm{t}, J=7.0$ $\left.\mathrm{Hz}, \quad 2 \mathrm{H}, \quad \mathrm{CH}_{3}(\mathrm{O}=\mathrm{C}) \mathrm{SC}_{2} \mathrm{CH}_{2}-\right), \quad 2.51-2.65 \quad\left(\mathrm{~m}, \quad 100 \mathrm{H}, \quad-\underline{\mathrm{C}}_{2} \mathrm{SC}_{2}-2, \quad-\underline{\mathrm{H}}_{2} \mathrm{SCH}-\right), \quad 2.35 \quad(\mathrm{~s}, \quad 3 \mathrm{H}$, $\left.\mathrm{C}_{3}(\mathrm{O}=\mathrm{C}-) \mathrm{SCH}_{2} \mathrm{CH}_{2}-\right), 1.87\left(\mathrm{~m}, 10 \mathrm{H},-\mathrm{CH}_{2} \underline{\mathrm{C}}_{2} \mathrm{CH}_{2} \mathrm{~S}-\right), 1.57\left(\mathrm{~m}, 24 \mathrm{H},-\mathrm{C}_{2} \mathrm{CH}_{2} \mathrm{CH}_{3}\right), 1.44(\mathrm{~m}, 24 \mathrm{H},-$ $\left.\mathrm{CH}_{2} \underline{\mathrm{C}}_{2} \mathrm{CH}_{3}\right), 1.03$ (m, 48H, $\left.-\mathrm{SiC}_{2} \mathrm{CH}_{2}-\right), 0.93$ (t, $J=7.0 \mathrm{~Hz}, 36 \mathrm{H},-\mathrm{CH}_{2} \mathrm{CH}_{2} \mathrm{C}_{3}$ ). 
p-C $\mathbf{C}_{3}$ D (139 mg, yield: 78\%). ${ }^{1} \mathrm{H}$ NMR $\left.400 \mathrm{MHz}, \mathrm{CDCl}_{3}, \mathrm{ppm}, \delta\right): 3.61-3.86\left(\mathrm{~m}, 24 \mathrm{H},-\mathrm{C} \underline{\mathrm{H}}(\mathrm{OH}) \underline{\mathrm{H}}_{2} \mathrm{OH}\right.$, -

$\left.\mathrm{SC} \underline{\mathrm{H}}(\mathrm{C}=\mathrm{O}) \mathrm{CH}_{2}-\right), 3.61\left(\mathrm{t}, J=7.0 \mathrm{~Hz}, 6 \mathrm{H},-\mathrm{NC}_{2} \mathrm{CH}_{2}-\right), 3.14,3.05\left(\mathrm{~m}, 6 \mathrm{H},-\mathrm{CHCH}_{2}(\mathrm{C}=\mathrm{O})-\right), 2.99(\mathrm{t}, J=7.0$

$\left.\mathrm{Hz}, \quad 2 \mathrm{H}, \quad \mathrm{CH}_{3}(\mathrm{O}=\mathrm{C}) \mathrm{SC}_{2} \mathrm{CH}_{2}-\right), \quad 2.51-2.63 \quad\left(\mathrm{~m}, \quad 134 \mathrm{H}, \quad-\underline{\mathrm{C}}_{2} \mathrm{SC}_{2}-2-\quad-\underline{\mathrm{H}}_{2} \mathrm{SCH}-\right), \quad 2.32 \quad(\mathrm{~s}, \quad 3 \mathrm{H}$, $\left.\mathrm{C}_{3}(\mathrm{O}=\mathrm{C}-) \mathrm{SCH}_{2} \mathrm{CH}_{2}-\right), 1.87\left(\mathrm{~m}, 14 \mathrm{H},-\mathrm{CH}_{2} \underline{\mathrm{C}}_{2} \mathrm{CH}_{2} \mathrm{~S}-\right), 1.53\left(\mathrm{~m}, 36 \mathrm{H},-\mathrm{C}_{2} \mathrm{CH}_{2} \mathrm{CH}_{3}\right), 1.47(\mathrm{~m}, 36 \mathrm{H},-$ $\left.\mathrm{CH}_{2} \underline{\mathrm{C}}_{2} \mathrm{CH}_{3}\right), 1.04\left(\mathrm{~m}, 64 \mathrm{H},-\mathrm{SiC}_{2} \mathrm{CH}_{2}-\right), 0.91\left(\mathrm{t}, J=7.0 \mathrm{~Hz}, 54 \mathrm{H},-\mathrm{CH}_{2} \mathrm{CH}_{2} \mathrm{C}_{3}\right)$.

p-C4 $\mathbf{D}$ (137 mg, yield: 77\%). ${ }^{1} \mathrm{H}$ NMR (400 MHz, $\left.\mathrm{CDCl}_{3}, \mathrm{ppm}, \delta\right): 3.67-3.81\left(\mathrm{~m}, 25 \mathrm{H},-\mathrm{C} \underline{\mathrm{H}}(\mathrm{OH}) \mathrm{CH}_{2} \mathrm{OH},-\right.$ $\left.\mathrm{SC} \underline{\mathrm{H}}(\mathrm{C}=\mathrm{O}) \mathrm{CH}_{2}-\right), 3.61\left(\mathrm{t}, J=7.0 \mathrm{~Hz}, 8 \mathrm{H},-\mathrm{NC}_{2} \mathrm{CH}_{2}-\right), 3.14,3.05\left(\mathrm{~m}, 8 \mathrm{H},-\mathrm{CHCH}_{2}(\mathrm{C}=\mathrm{O})-\right), 2.92(\mathrm{t}, J=7.0$ $\left.\mathrm{Hz}, \quad 2 \mathrm{H}, \quad \mathrm{CH}_{3}(\mathrm{O}=\mathrm{C}) \mathrm{SC}_{2} \mathrm{CH}_{2}-\right), \quad 2.51-2.65 \quad\left(\mathrm{~m}, \quad 168 \mathrm{H}, \quad-\underline{\mathrm{C}}_{2} \mathrm{SC}_{2}-2-\quad-\underline{\mathrm{H}}_{2} \mathrm{SCH}-\right), \quad 2.39 \quad(\mathrm{~s}, \quad 3 \mathrm{H}$, $\left.\mathrm{C}_{3}(\mathrm{O}=\mathrm{C}-) \mathrm{SCH}_{2} \mathrm{CH}_{2}-\right), 1.81\left(\mathrm{~m}, 18 \mathrm{H},-\mathrm{CH}_{2} \underline{\mathrm{C}}_{2} \mathrm{CH}_{2} \mathrm{~S}-\right), 1.54\left(\mathrm{~m}, 48 \mathrm{H},-\mathrm{C}_{2} \mathrm{CH}_{2} \mathrm{CH}_{3}\right), 1.47(\mathrm{~m}, 48 \mathrm{H},-$ $\left.\mathrm{CH}_{2} \underline{\mathrm{C}}_{2} \mathrm{CH}_{3}\right), 1.03$ (m, 80H, $\left.-\mathrm{SiC}_{2} \mathrm{CH}_{2}-\right), 0.97$ (t, $J=7.0 \mathrm{~Hz}, 72 \mathrm{H},-\mathrm{CH}_{2} \mathrm{CH}_{2} \mathrm{C}_{3}$ ).

p-C5 $\mathbf{D}$ (129 mg, yield: 75\%). ${ }^{1} \mathrm{H}$ NMR (400 MHz, $\left.\mathrm{CDCl}_{3}, \mathrm{ppm}, \delta\right): 3.71-3.82\left(\mathrm{~m}, 26 \mathrm{H},-\mathrm{C} \underline{\mathrm{H}}(\mathrm{OH}) \mathrm{C}_{2} \mathrm{OH},-\right.$ $\left.\mathrm{SC} \underline{\mathrm{H}}(\mathrm{C}=\mathrm{O}) \mathrm{CH}_{2}-\right), 3.63\left(\mathrm{t}, J=7.0 \mathrm{~Hz}, 10 \mathrm{H},-\mathrm{NC}_{2} \mathrm{CH}_{2}-\right), 3.14,3.05\left(\mathrm{~m}, 10 \mathrm{H},-\mathrm{CHCH}_{2}(\mathrm{C}=\mathrm{O})-\right), 2.98(\mathrm{t}, J=$ $\left.7.0 \mathrm{~Hz}, \quad 2 \mathrm{H}, \quad \mathrm{CH}_{3}(\mathrm{O}=\mathrm{C}) \mathrm{SC}_{2} \mathrm{CH}_{2}-\right), 2.51-2.65$ (m, 102H, $\left.-\underline{\mathrm{C}}_{2} \mathrm{SC}_{2}-2,-\underline{\mathrm{C}}_{2} \mathrm{SCH}-\right), 2.35$ (s, 3H, $\left.\mathrm{C}_{3}(\mathrm{O}=\mathrm{C}-) \mathrm{SCH}_{2} \mathrm{CH}_{2}-\right), 1.86\left(\mathrm{~m}, 22 \mathrm{H},-\mathrm{CH}_{2} \underline{\mathrm{C}}_{2} \mathrm{CH}_{2} \mathrm{~S}-\right), 1.57\left(\mathrm{~m}, 60 \mathrm{H},-\mathrm{C}_{2} \mathrm{CH}_{2} \mathrm{CH}_{3}\right), 1.43(\mathrm{~m}, 60 \mathrm{H},-$ $\left.\mathrm{CH}_{2} \mathrm{C}_{2} \mathrm{CH}_{3}\right), 1.03\left(\mathrm{~m}, 96 \mathrm{H},-\mathrm{SiC}_{2} \mathrm{CH}_{2}-\right), 0.93\left(\mathrm{t}, J=7.0 \mathrm{~Hz}, 90 \mathrm{H},-\mathrm{CH}_{2} \mathrm{CH}_{2} \mathrm{C}_{3}\right)$.

p-C6 $\mathbf{D}$ (122 mg, yield: 72\%). ${ }^{1} \mathrm{H}$ NMR (400 MHz, $\left.\mathrm{CDCl}_{3}, \mathrm{ppm}, \delta\right): 3.71-3.82\left(\mathrm{~m}, 27 \mathrm{H},-\mathrm{C} \underline{\mathrm{H}}(\mathrm{OH}) \mathrm{CH}_{2} \mathrm{OH},-\right.$ $\left.\mathrm{SC} \underline{\mathrm{H}}(\mathrm{C}=\mathrm{O}) \mathrm{CH}_{2}-\right), 3.64\left(\mathrm{t}, J=7.0 \mathrm{~Hz}, 12 \mathrm{H},-\mathrm{NC}_{2} \mathrm{CH}_{2}-\right), 3.14,3.05\left(\mathrm{~m}, 12 \mathrm{H},-\mathrm{CHCH}_{2}(\mathrm{C}=\mathrm{O})-\right), 2.98(\mathrm{t}, J=$ $\left.7.0 \mathrm{~Hz}, 2 \mathrm{H}, \quad \mathrm{CH}_{3}(\mathrm{O}=\mathrm{C}) \mathrm{SC}_{2} \mathrm{CH}_{2}-\right), 2.51-2.65$ (m, 236H, $\left.-\underline{\mathrm{CH}}_{2} \mathrm{SC}_{2}-2,-\underline{\mathrm{C}}_{2} \mathrm{SCH}-\right), 2.36$ (s, 3H, $\left.\mathrm{C}_{3}(\mathrm{O}=\mathrm{C}-) \mathrm{SCH}_{2} \mathrm{CH}_{2}-\right), 1.83\left(\mathrm{~m}, 26 \mathrm{H},-\mathrm{CH}_{2} \underline{\mathrm{C}}_{2} \mathrm{CH}_{2} \mathrm{~S}-\right), 1.55\left(\mathrm{~m}, 72 \mathrm{H},-\mathrm{C}_{2} \mathrm{CH}_{2} \mathrm{CH}_{3}\right), 1.42(\mathrm{~m}, 72 \mathrm{H},-$ $\mathrm{CH}_{2} \underline{\mathrm{C}}_{2} \mathrm{CH}_{3}$ ), 1.07 (m, 112H, $\left.-\mathrm{SiC}_{2} \mathrm{CH}_{2}-\right), 0.93$ (t, $J=7.0 \mathrm{~Hz}, 108 \mathrm{H},-\mathrm{CH}_{2} \mathrm{CH}_{2} \mathrm{C}_{3}$ ).

p-C8D (119 mg, yield: 70\%). ${ }^{1} \mathrm{H}$ NMR (400 MHz, $\left.\mathrm{CDCl}_{3}, \mathrm{ppm}, \delta\right): 3.71-3.82\left(\mathrm{~m}, 29 \mathrm{H},-\mathrm{C} \underline{\mathrm{H}}(\mathrm{OH}) \underline{\mathrm{H}}_{2} \mathrm{OH},-\right.$ $\left.\mathrm{SC} \underline{\mathrm{H}}(\mathrm{C}=\mathrm{O}) \mathrm{CH}_{2}-\right), 3.63\left(\mathrm{t}, J=7.0 \mathrm{~Hz}, 16 \mathrm{H},-\mathrm{NC}_{2} \mathrm{CH}_{2}-\right), 3.14,3.05\left(\mathrm{~m}, 16 \mathrm{H},-\mathrm{CHCH}_{2}(\mathrm{C}=\mathrm{O})-\right), 2.98(\mathrm{t}, J=$ $\left.7.0 \mathrm{~Hz}, 2 \mathrm{H}, \quad \mathrm{CH}_{3}(\mathrm{O}=\mathrm{C}) \mathrm{SCH}_{2} \mathrm{CH}_{2}-\right), 2.51-2.65$ (m, 304H, - $\left.\underline{\mathrm{C}}_{2} \mathrm{SC}_{2}-2,-\underline{\mathrm{C}}_{2} \mathrm{SCH}-\right), 2.35$ (s, 3H, 
$\left.\mathrm{C}_{3}(\mathrm{O}=\mathrm{C}-) \mathrm{SCH}_{2} \mathrm{CH}_{2}-\right), 1.86\left(\mathrm{~m}, 34 \mathrm{H},-\mathrm{CH}_{2} \underline{\mathrm{C}}_{2} \mathrm{CH}_{2} \mathrm{~S}-\right), 1.57\left(\mathrm{~m}, 96 \mathrm{H},-\mathrm{C}_{2} \mathrm{CH}_{2} \mathrm{CH}_{3}\right), 1.43(\mathrm{~m}, 96 \mathrm{H},-$ $\left.\mathrm{CH}_{2} \underline{\mathrm{C}}_{2} \mathrm{CH}_{3}\right), 1.03\left(\mathrm{~m}, 144 \mathrm{H},-\mathrm{SiC}_{2} \mathrm{CH}_{2}-\right), 0.93$ (t, $\left.J=7.0 \mathrm{~Hz}, 144 \mathrm{H},-\mathrm{CH}_{2} \mathrm{CH}_{2} \mathrm{CH}_{3}\right)$.

\subsection{Discrete oDMS-DPOSS Conjugate $\left(\mathrm{S}_{13} \mathrm{D}\right)$}

Cl-Si4-H (128 g, yiled: 75\%): Hexamethylcyclotrisiloxane (120 g, 0.54 mol), dimethylchlorosilane (51 g, 0.54 $\mathrm{mol})$ and acetonitrile $(25 \mathrm{~mL})$ was mixed in a $500 \mathrm{ml}$ round-bottom flask in a glove box. DMF $(1.5 \mathrm{~mL})$ was then added and the mixture was stirred at room temperature for $72 \mathrm{~h}$. The pure product was obtained by vacuum distillation (the fraction $40-41^{\circ} \mathrm{C}$ ) as a colorless oil (128 g, 75\%).

General synthetic procedure for $\mathbf{S}_{\mathbf{x}-4}-\mathbf{O H}$ : Taking $\mathrm{S}_{8}-\mathrm{OH}$ as an example. In a $250 \mathrm{~mL}$ round-bottom flask, $\mathrm{Pd} / \mathrm{C}(0.99 \mathrm{~g}, 0.93 \mathrm{mmol}$ of $\mathrm{Pd})$ was dispersed in a mixture of dioxane $(70 \mathrm{~mL})$ and $1 \mathrm{M}$ phosphate buffer $(\mathrm{pH}$ $=7,15 \mathrm{~mL})$. A solution of $\mathrm{S}_{8}(34.1 \mathrm{~g}, 46.8 \mathrm{mmol})$ in dioxane $(35 \mathrm{~mL})$ was added dropwise to the mixture under ice bath. The solution was then further stirred for another $4 \mathrm{~h}$ at room temperature. The mixture was filtrated to remove $\mathrm{Pd} / \mathrm{C}$. The aqueous layer was separated and extracted with toluene $(100 \mathrm{~mL})$. The organic phases were combined and washed with water $(3 \times 100 \mathrm{~mL})$ and dried with anhydrous $\mathrm{MgSO}_{4}$. Toluene were removed in vacuo to afford $\mathrm{S}_{8}-\mathrm{OH}$ as a colorless oil $(>95 \%)$.

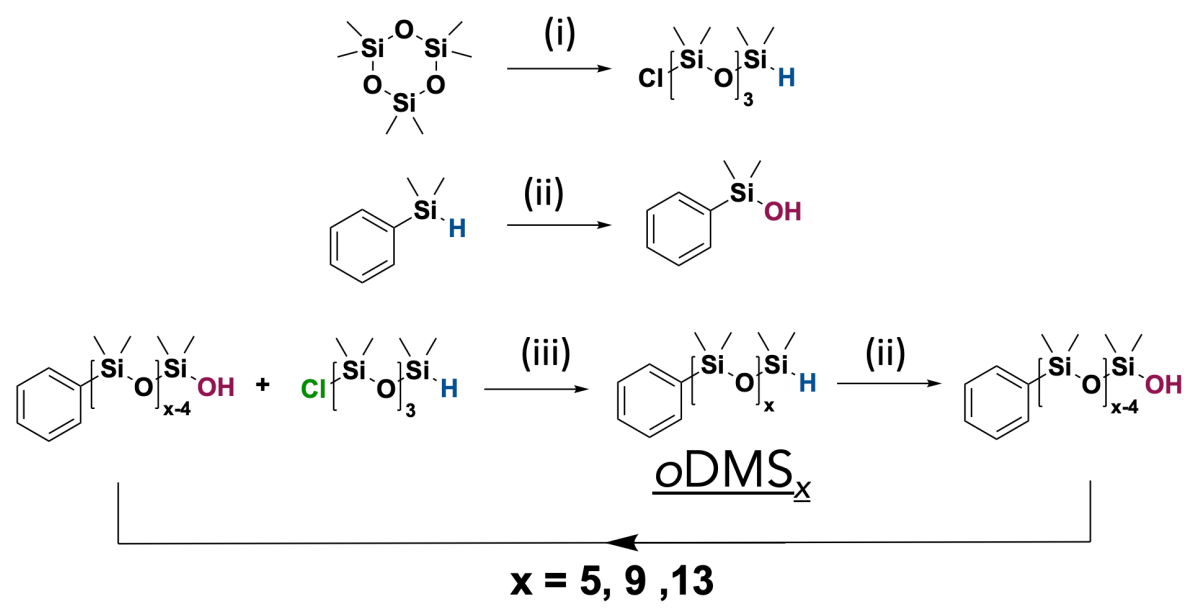

Scheme S6. Synthetic Route of discrete $o \mathrm{DMS}$ : (i) $\mathrm{MeSiClH}, \mathrm{CH}_{3} \mathrm{CN}$, DMF; (ii) $\mathrm{Pd} / \mathrm{C}, 1 \mathrm{M} \mathrm{PB}$ (pH = 7), dioxane; (iii) pyridine, toluene. 
S5-OH (39 g, yield: 98\%). ${ }^{1} \mathrm{H}$ NMR (400 MHz, $\mathrm{CDCl}_{3}, \mathrm{ppm}, \delta$ ): 7.35-7.58 (m, 5H, Ar- $\left.\underline{\mathrm{H}}\right), 0.34$ (d, J=6.1 Hz, 6H, $\left.\operatorname{ArSi}\left(\underline{\mathrm{CH}}_{3}\right)_{2}-\right), 0.15\left(\mathrm{~d}, J=3.2 \mathrm{~Hz}, 6 \mathrm{H},-\mathrm{Si}\left(\mathrm{C}_{3}\right)_{2} \mathrm{OH}\right), 0.07$ (m, 18H, $\left.-\mathrm{Si}\left(\mathrm{C}_{\mathrm{H}_{3}}\right)_{2}{ }^{-}\right)$.

S9-OH (52g, yield: 98\%). ${ }^{1} \mathrm{H}$ NMR (400 MHz, $\mathrm{CDCl}_{3}$, ppm, $\delta$ ): 7.35-7.58 (m, 5H, Ar- $\left.\underline{\mathrm{H}}\right), 0.34$ (d, $J=5.9 \mathrm{~Hz}$, $\left.6 \mathrm{H}, \operatorname{ArSi}\left(\underline{\mathrm{CH}}_{3}\right)_{2}-\right), 0.15\left(\mathrm{~d}, J=2.9 \mathrm{~Hz}, 6 \mathrm{H},-\mathrm{Si}\left(\mathrm{C}_{3}\right)_{2} \mathrm{OH}\right), 0.07\left(\mathrm{~m}, 66 \mathrm{H},-\mathrm{Si}\left(\mathrm{CH}_{3}\right)_{2-}\right)$.

General procedure for synthesis of $\mathbf{S}_{\mathbf{x}}$ : Taking $\mathrm{S}_{13}$ as an example. In a glove box, Cl-Si4-H $(14.5 \mathrm{~g}, 45.6$ $\mathrm{mmol})$ was dissolved in toluene $(100 \mathrm{~mL})$ in a $500 \mathrm{ml}$ round-bottom flask. $\mathrm{S}_{9}-\mathrm{OH}(34.0 \mathrm{~g}, 45.6 \mathrm{mmol})$ was added dropwise into the mixture in $30 \mathrm{~min}$ at $0{ }^{\circ} \mathrm{C}$. The mixture was further stirred for another 4 hours. The reaction mixture was quenched by adding water $(100 \mathrm{~mL})$ and toluene $(100 \mathrm{~mL})$. The organic phases were collected and washed with water $(3 \times 100 \mathrm{ml})$ and dried with anhydrous $\mathrm{MgSO}_{4}$. Toluene was removed in vacuo to give the crude product, which was purified by vacuum distillation (fraction $200-205{ }^{\circ} \mathrm{C}$ ) to afford $\mathrm{S}_{13}$ as a colorless oil $(35.2 \mathrm{~g}, 75 \%)$.

S5-H (40.5 g, yield: 85\%). ${ }^{1} \mathrm{H}$ NMR (400 MHz, $\left.\mathrm{CDCl}_{3}, \mathrm{ppm}, \delta\right): 7.34-7.57$ (m, 5H, Ar- $\left.\underline{\mathrm{H}}\right), 4.70(\mathrm{p}, J=2.1$ $\left.\mathrm{Hz}, 1 \mathrm{H},-\mathrm{Si}\left(\mathrm{CH}_{3}\right)_{2} \underline{\mathrm{H}}\right), 0.34\left(\mathrm{~d}, J=6.5 \mathrm{~Hz}, 6 \mathrm{H}, \operatorname{ArSi}\left(\mathrm{C}_{3}\right)_{2}-\right), 0.19\left(\mathrm{~d}, J=2.9 \mathrm{~Hz}, 6 \mathrm{H}, \mathrm{HSi}\left(\mathrm{C}_{3}\right)_{2}-\right), 0.07(\mathrm{~m}$, $\left.18 \mathrm{H},-\mathrm{Si}\left(\mathrm{CH}_{3}\right)_{2}-\right)$.

S9-H (53.1 g, yield: 80\%). ${ }^{1} \mathrm{H}$ NMR (400 MHz, $\mathrm{CDCl}_{3}$, ppm, $\delta$ ): 7.34-7.57 (m, 5H, Ar- $\left.\underline{\mathrm{H}}\right), 4.70$ (p, $J=2.4 \mathrm{~Hz}$, 1H, $\left.-\mathrm{Si}\left(\mathrm{CH}_{3}\right)_{2} \underline{\mathrm{H}}\right), 0.34\left(\mathrm{~d}, J=6.2 \mathrm{~Hz}, 6 \mathrm{H}, \operatorname{ArSi}\left(\underline{\mathrm{C}}_{3}\right)_{2}-\right), 0.19\left(\mathrm{~d}, J=2.9 \mathrm{~Hz}, 6 \mathrm{H}, \mathrm{HSi}\left(\mathrm{C}_{3}\right)_{2}-\right), 0.07(\mathrm{~m}, 48 \mathrm{H}$, $\left.-\mathrm{Si}\left(\underline{\mathrm{C}}_{3}\right)_{2}-\right)$.

S13-H (35.2 g, yield: 75\%). ${ }^{1} \mathrm{H}$ NMR (400 MHz, $\left.\mathrm{CDCl}_{3}, \mathrm{ppm}, \delta\right): 7.34-7.57$ (m, 5H, Ar- $\left.\underline{\mathrm{H}}\right), 4.70(\mathrm{p}, J=2.8$ $\left.\mathrm{Hz}, 1 \mathrm{H},-\mathrm{Si}\left(\mathrm{CH}_{3}\right)_{2} \underline{\mathrm{H}}\right), 0.34\left(\mathrm{~d}, J=6.9 \mathrm{~Hz}, 6 \mathrm{H}, \mathrm{ArSi}\left(\mathrm{CH}_{3}\right)_{2}-\right), 0.19\left(\mathrm{~d}, J=2.8 \mathrm{~Hz}, 6 \mathrm{H}, \mathrm{HSi}\left(\mathrm{C}_{3}\right)_{2}-\right), 0.07(\mathrm{~m}$, $\left.66 \mathrm{H},-\mathrm{Si}\left(\mathrm{C}_{3}\right)_{2}-\right)$. 


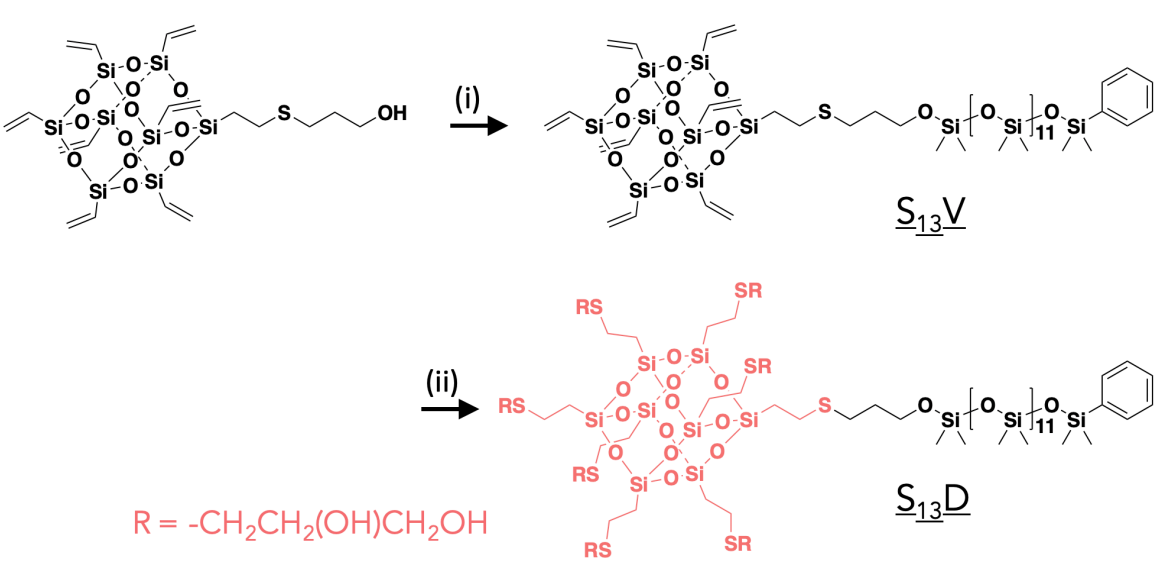

Scheme S7. Synthetic Route of discrete $\mathrm{S}_{13} \mathrm{D}$ : (i) $\mathrm{S}_{13}-\mathrm{H}, \mathrm{B}\left(\mathrm{C}_{6} \mathrm{~F}_{5}\right)_{3}$, DCM; (ii) 1-thioglycerol, AIBN, toluene.

$\mathbf{S}_{13} \mathbf{V}$ : In a glove box, $\mathrm{S}_{13}(0.20 \mathrm{~g}, 0.20 \mathrm{mmol})$ and VPOSS-OH $(0.16 \mathrm{~g}, 0.22 \mathrm{mmol})$ were dissolved in $\mathrm{CH}_{2} \mathrm{Cl}_{2}$ ( $3 \mathrm{~mL})$ in $25 \mathrm{~mL}$ round-bottom flask. A solution of $\mathrm{B}\left(\mathrm{C}_{6} \mathrm{~F}_{5}\right)_{3}(20 \mathrm{mg}, 0.04 \mathrm{mmol})$ in $\mathrm{CH}_{2} \mathrm{Cl}_{2}(1 \mathrm{~mL})$ was added quickly into the mixture. The mixture was stirred for $15 \mathrm{~min}$ at room temperature, and then quenched by adding water $(20 \mathrm{~mL})$ and $\mathrm{CH}_{2} \mathrm{Cl}_{2}(50 \mathrm{~mL})$. The organic phases were washed with water $(2 \times 50 \mathrm{ml})$ and dried with $\mathrm{MgSO}_{4} . \mathrm{CH}_{2} \mathrm{Cl}_{2}$ was removed in vacuo to give the crude product, which was further purified by recycling preparative SEC to give $\mathrm{S}_{13} \mathrm{~V}$ as clear, waxy solid (0.29 g, yield: $\left.80 \%\right) .{ }^{1} \mathrm{H} \mathrm{NMR}\left(400 \mathrm{MHz}, \mathrm{CDCl}_{3}, \mathrm{ppm}, \delta\right)$ : 7.34-7.57 (m, 5H, Ar- $\underline{\mathrm{H}}), 5.86-6.13\left(\mathrm{~m}, 21 \mathrm{H}, \mathrm{C}_{2}=\mathrm{C} \underline{\mathrm{H}}-\right), 3.73$ (t, $\left.J=7.0 \mathrm{~Hz}, 2 \mathrm{H},-\mathrm{CH}_{2} \mathrm{C}_{2} \mathrm{OSi}\left(\mathrm{CH}_{3}\right)_{2}-\right), 2.57-$ $2.65\left(\mathrm{~m}, 4 \mathrm{H},-\mathrm{CH}_{2} \mathrm{CH}_{2} \mathrm{SCH}_{2} \mathrm{CH}_{2}-\right), 1.79\left(\mathrm{tt}, J=7.0,6.1 \mathrm{~Hz}, 2 \mathrm{H},-\mathrm{SCH}_{2} \underline{\mathrm{C}}_{2} \mathrm{CH}_{2} \mathrm{~S}\left(\mathrm{CH}_{3}\right)_{2}-\right), 1.07(\mathrm{~m}, 2 \mathrm{H},-$ $\left.\mathrm{SiC}_{2} \mathrm{CH}_{2} \mathrm{~S}-\right), 0.34$ (d, $\left.J=6.9 \mathrm{~Hz}, 6 \mathrm{H}, \operatorname{ArSi}\left(\mathrm{C}_{3}\right)_{2}-\right), 0.05-0.09$ (m, 72H, $\left.-\mathrm{Si}\left(\mathrm{C}_{3}\right)_{2}-\right)$.

S13D: In a glove box, $\mathrm{S}_{13} \mathrm{~V}(0.10 \mathrm{~g}, 0.057 \mathrm{mmol})$, 1-thioglycerol (0.0867 g, $\left.0.802 \mathrm{mmol}\right)$, and AIBN (0.0263 $\mathrm{g}, 0.160 \mathrm{mmol})$ were dissolved in toluene $(5 \mathrm{~mL})$ in $25 \mathrm{~mL}$ round-bottom flask. The mixture was moved out of box and heated to $75{ }^{\circ} \mathrm{C}$ for $24 \mathrm{~h}$. Toluene was removed in vacuo to give the crude product, which was purified by recycling preparative SEC to give $\mathrm{S}_{13} \mathrm{D}$ as waxy solid $(0.10 \mathrm{~g}$, yield: $65 \%) .{ }^{1} \mathrm{H}$ NMR (400 MHZ, DMSO, ppm, $\delta): ~ 7.31-7.51(\mathrm{~m}, 5 \mathrm{H}, \mathrm{Ar}-\underline{\mathrm{H}}), 4.51-4.73\left(\mathrm{~m}, 14 \mathrm{H},-\mathrm{CH}_{2} \mathrm{CH}(\mathrm{O} \underline{\mathrm{H}}) \mathrm{CH}_{2}(\mathrm{O} \underline{\mathrm{H}})\right), 3.65(\mathrm{t}, J=7.9 \mathrm{~Hz}$, $\left.2 \mathrm{H},-\mathrm{CH}_{2} \underline{\mathrm{CH}}_{2} \mathrm{OSi}\left(\mathrm{CH}_{3}\right)_{2}-\right), 3.52\left(\mathrm{~m}, 7 \mathrm{H},-\mathrm{CH}_{2} \mathrm{C} \underline{\mathrm{H}}(\mathrm{OH}) \mathrm{C}_{2}(\mathrm{OH})\right), 2.39-2.62\left(\mathrm{~m}, 18 \mathrm{H},-\mathrm{CH}_{2} \underline{\mathrm{C}}_{2} \mathrm{SC}_{2} \mathrm{CH}_{2}-,-\right.$ $\left.\mathrm{C}_{2} \mathrm{CH}(\mathrm{OH}) \mathrm{CH}_{2}(\mathrm{OH})\right), 1.66\left(\mathrm{tt}, J=7.0,6.1 \mathrm{~Hz}, 2 \mathrm{H},-\mathrm{SCH}_{2} \underline{\mathrm{C}}_{2} \mathrm{CH}_{2} \mathrm{~S}\left(\mathrm{CH}_{3}\right)_{2}-\right), 0.95\left(\mathrm{~m}, 16 \mathrm{H},-\mathrm{SiCH}_{2} \mathrm{CH}_{2} \mathrm{~S}-\right.$, 
$\left.-\mathrm{SiCH}_{2} \mathrm{CH}_{2} \mathrm{SCH}_{2} \mathrm{CH}(\mathrm{OH}) \mathrm{CH}_{2}(\mathrm{OH})\right), 0.28\left(\mathrm{~d}, J=7.3 \mathrm{~Hz}, 6 \mathrm{H}, \operatorname{ArSi}\left(\mathrm{C}_{3}\right)_{2}-\right), 0.01-0.02\left(\mathrm{~m}, 72 \mathrm{H},-\mathrm{Si}\left(\mathrm{C}_{3}\right)_{2}-\right)$.

\section{Detailed Synthesis and Characterizations.}

4.1 Preparation of Amphiphilic Block Chains $\left(\boldsymbol{p}-\mathbf{C}_{n} \mathbf{D}\right)$. To conjugate DPOSS moiety onto CPOSS chains, a thiol-functionalized VPOSS (V7POSS-SH) was first prepared (Scheme S4), and then coupled with activated $\mathrm{C}_{n}$ blocks through Michael coupling reaction (labeled as $p-\mathrm{C}_{n} \mathrm{~V}$ ). Further decorating the POSS cage with 14 hydroxyl groups via thiol-ene reaction leads to the amphiphilic block chains ( $p-\mathrm{C}_{n} \mathrm{D}$, Scheme S5). Here we take $p-\mathrm{C}_{3} \mathrm{D}$ as an example to illustrate the synthetic details. After coupling, the SEC trace of $p-\mathrm{C}_{3} \mathrm{~V}$ shifts clearly towards low retention volume, as compared with the precursor $p$ - $\mathrm{C}_{3}$ (Figure $\mathrm{S} 17 \mathrm{~b}$ ). A single peak was observed in the corresponding MALDI-ToF MS, with molecular mass (5159.43 Da) in good agreement with calculated value $(5158.82 \mathrm{Da})$, confirming the proposed chemical structure with atomic accuracy (Figure S17c). The ${ }^{1} \mathrm{H}$ NMR spectrum of the resultant product $\left(p-\mathrm{C}_{3} \mathrm{~V}\right)$ contains signals from both components (Figure $\left.\mathrm{S} 17 \mathrm{a}\right)$. In particular, the typical resonances of vinyl groups appear at 5.8-6.2 ppm. Installation of surface functionalities onto the vinyl groups $\left(p-\mathrm{C}_{n} \mathrm{D}\right)$ proceeds promptly and quantitatively under UV irradiation. As expected, the signals attributed to the vinyl groups disappeared completely, while the resonances of thioglycerol emerge $(\delta$ 3.85, 3.12, and $2.74 \mathrm{ppm}$, Figure S17a). A further shift of the SEC trace confirms the increase of the hydrodynamic volume (Figure S17b). Due to collective hydrogen bonds, partial aggregation exists in the solution among DPOSS cages, as indicated by a shoulder peak in the SEC trace. Due to low ionization efficiency, acquiring a high-quality mass spectrum of $p-\mathrm{C}_{3} \mathrm{D}$ turned out to be very challenging. Similar situations have also been reported in the literatures. ${ }^{[1]}$ Nevertheless, other complementary characterizations (i.e. NMR and SEC) could provide convincing evidences towards the success of the synthesis. Amphiphilic block chains with varied compositions can be modularly synthesized using corresponding precursors (Figures S18-S20). The molecular 
characterizations of this series of discrete molecules are summarized in Table 2.

4.2 Preparation of Particle-Polymer Conjugate $\left(\mathbf{S}_{13} \mathbf{D}\right)$. Discrete $o$ DMS was synthesized following an iterative exponential growth approach according to literature with slight modification (Scheme S6). ${ }^{[2]}$ Endcapping with a VPOSS moeity, and further decorating the periphery of the particle with thioglycerol ligands through thiol-ene reaction leads to the linear polymer-particle conjugate $\left(\mathrm{S}_{13} \mathrm{D}\right.$, Scheme $\left.\mathrm{S} 7\right)$. The chemical structure and discrete feature were fully characterized by a combination of NMR, SEC, and MALDI-ToF MS (Figure S25).

\section{Equation and calculations}

\subsection{Volume fraction of CPOSS $\left(f_{\mathrm{C}}\right)$.}

The volume fraction of CPOSS can be calculated by Eq. S1:

$$
f_{A}=\frac{n * M_{C} / \rho_{C}}{n * M_{C} / \rho_{C}+M_{D} / \rho_{D}}
$$

where $M_{\mathrm{D}}$ is the molecular weight of DPOSS (1430.2 $\left.\mathrm{g} / \mathrm{mol}\right), M_{\mathrm{C}}$ is the molecular weight of CPOSS (1453.6 $\mathrm{g} / \mathrm{mol}), n$ is the number of CPOSS, and $\rho_{\mathrm{D}}$ and $\rho_{\mathrm{C}}$ are the density of DPOSS and CPOSS, respectively. The density of DPOSS is measured to be around $1.43 \mathrm{~g} / \mathrm{cm}^{3}$, and the density of CPOSS is estimated to be around $1.05 \mathrm{~g} / \mathrm{cm}^{3}$ (assuming the density of CPOSS is similar to that of BPOSS, i.e., POSS with seven isobutyl groups $)^{[3-4]}$.

\subsection{Domain spacing $(d)$.}

The domain spacing $(d)$ is calculated directed from the primary scattering peak $\left(q_{1}\right)$ of the SAXS profile:

$$
d=\frac{2 \pi}{q_{1}}
$$




\subsection{Characteristic dimension of the phase $(a)$.}

The dimension of the phase $(a)$ refers to lamellar periodicities (for LAM), inter-column distances (for HEX), and lattice parameters (for DG and BCC). It can be calculated from domain spacing $(d)$ accordingly.

$\begin{array}{cc}a=d & \text { for LAM } \\ a=\sqrt{6} d & \text { for DG } \\ a=2 d / \sqrt{3} & \text { for HEX } \\ a=\sqrt{2} d & \text { for BCC }\end{array}$

\subsection{Volume of the monomers}

The volume of the monomer can be estimated by the following calculation: ${ }^{5]}$

$$
V=\frac{M}{N_{A} \rho}
$$

where $M$ and $\rho$ are the molecular weight and density of the monomer. $N_{\mathrm{A}}$ is the Avogadro constant. According to literature, $M_{\mathrm{CPOSS}}=1457.6 \mathrm{~g} / \mathrm{mol}, M_{\mathrm{oDMS}}=74 \mathrm{~g} / \mathrm{mol} ; \rho_{\mathrm{CPOSS}}=1.05 \mathrm{~g} / \mathrm{cm}^{3}, \rho_{\mathrm{oDMS}}=0.95$ $\mathrm{g} / \mathrm{cm}^{3}$. Plug in the relevant parameters to get: $V_{\mathrm{CPOSS}}=2.3 \mathrm{~nm}^{3}, V_{\mathrm{oDMS}}=0.13 \mathrm{~nm}^{3}$. 


\section{Supplemental Schemes and Figures}
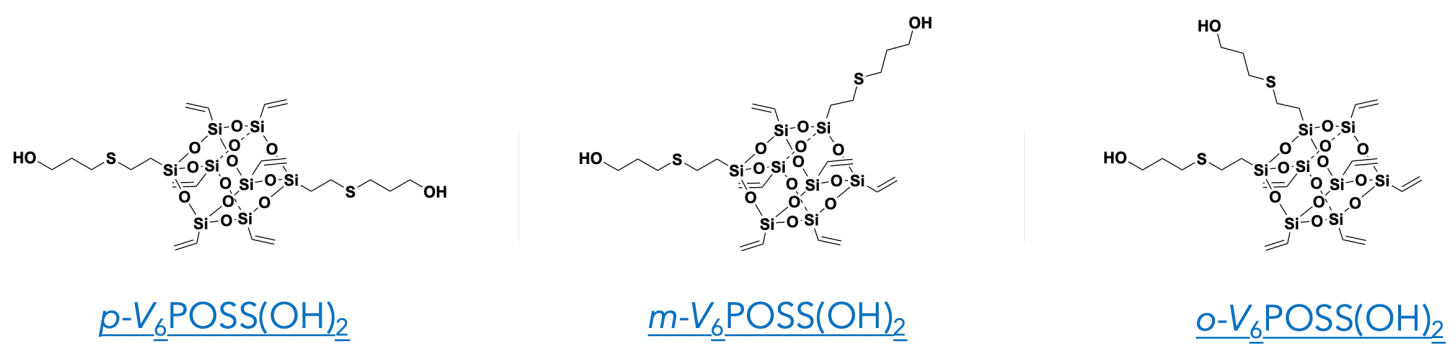

Figure S1. Regioisomers of octavinyl POSS with two hydroxyl substituting groups at para-, meta-, and ortho-positions.
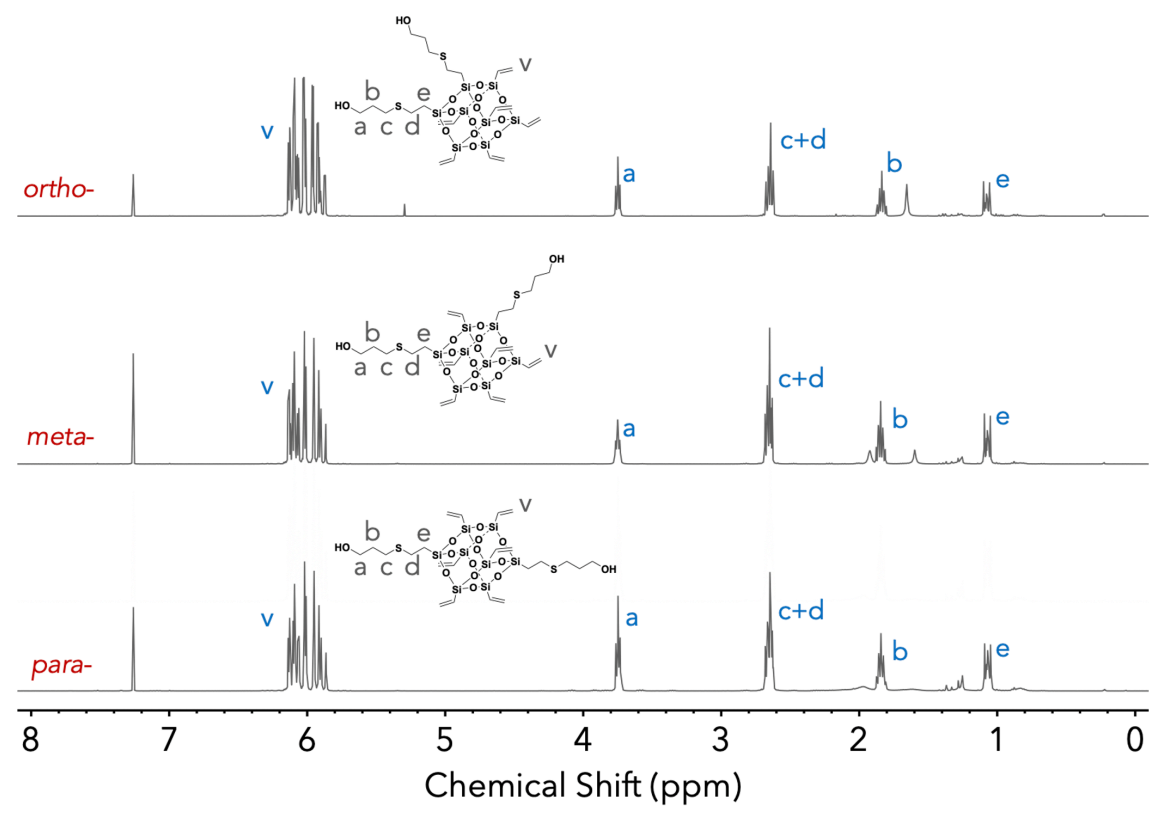

Figure S2. ${ }^{1} \mathrm{H}$ NMR spectra of $p-, m-$, and $o-\mathrm{V}_{6} \mathrm{POSS}(\mathrm{OH})_{2}$. 


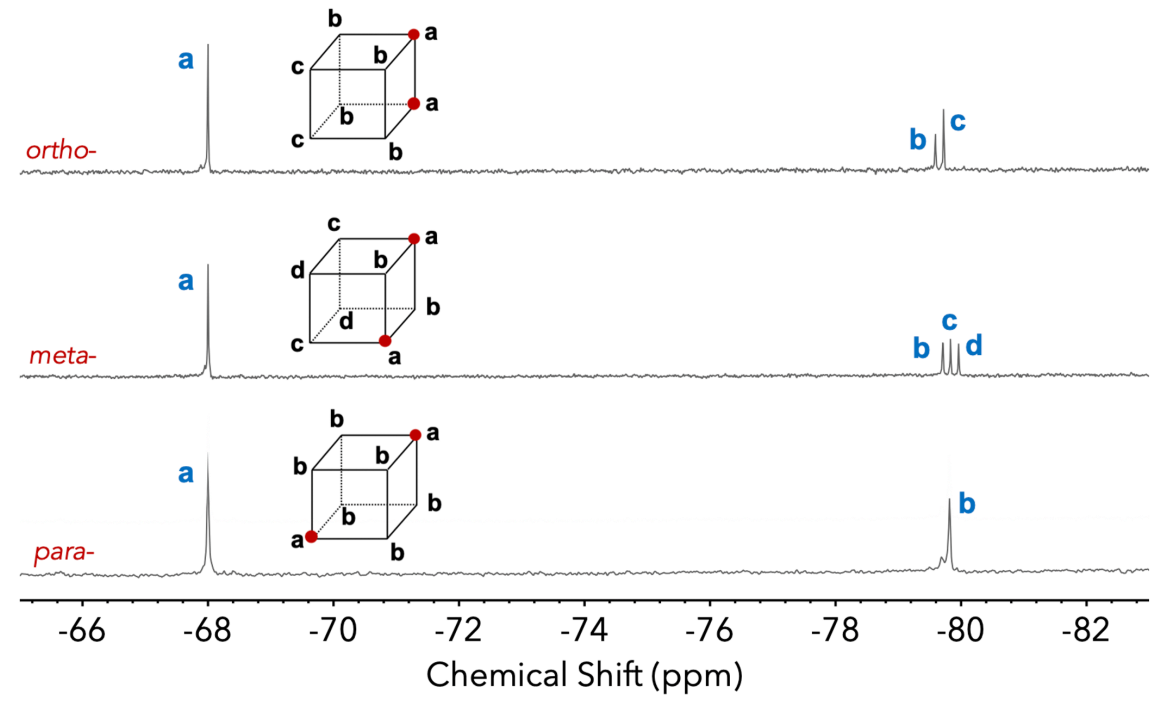

Figure S3. ${ }^{29} \mathrm{Si} \mathrm{NMR}$ spectra of $p-, m-$, and $o-\mathrm{V}_{6} \mathrm{POSS}(\mathrm{OH})_{2}$.

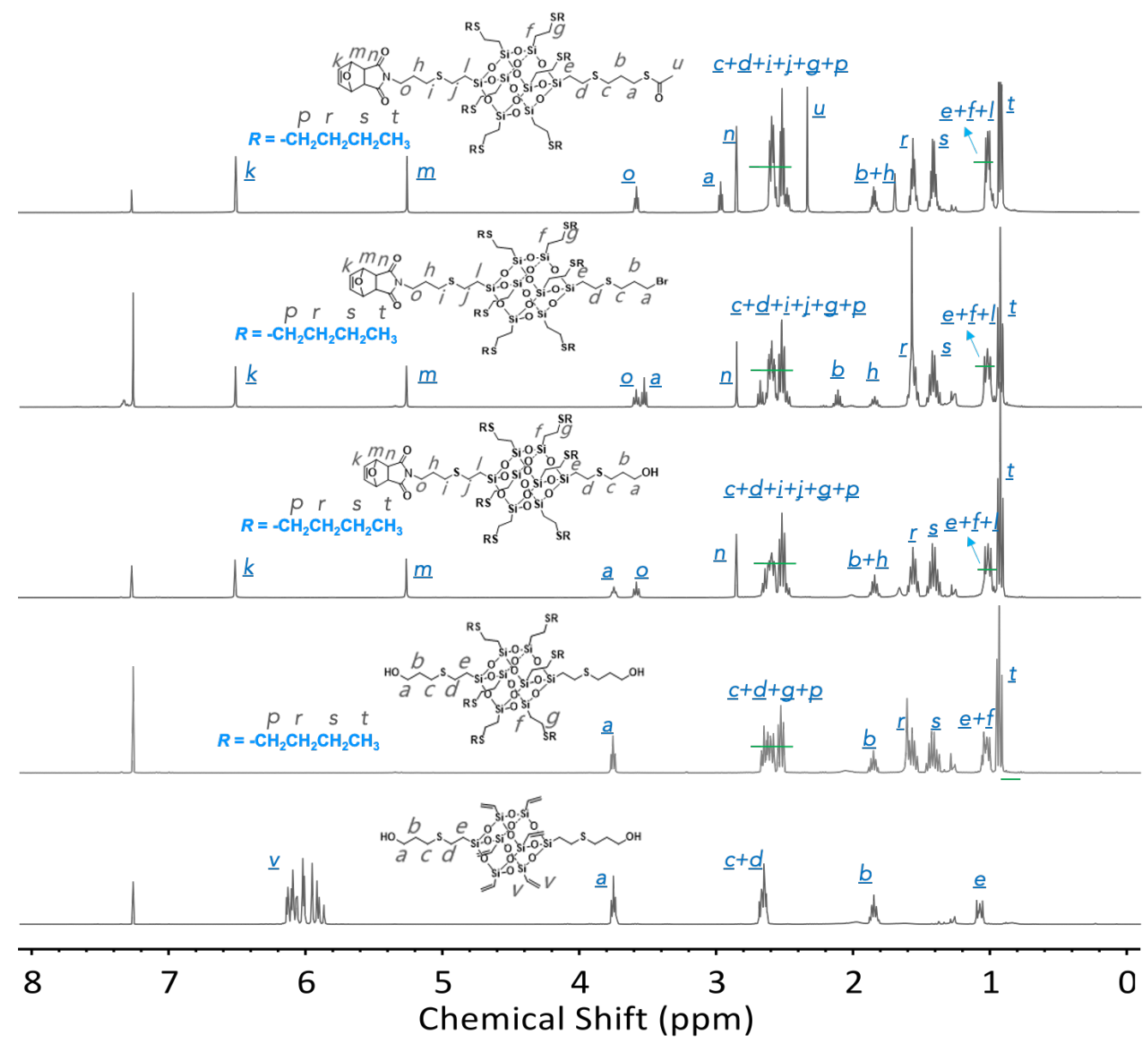

Figure S4. ${ }^{1} \mathrm{H}$ NMR spectra of the intermediates of $p$-CPOSS monomer. 

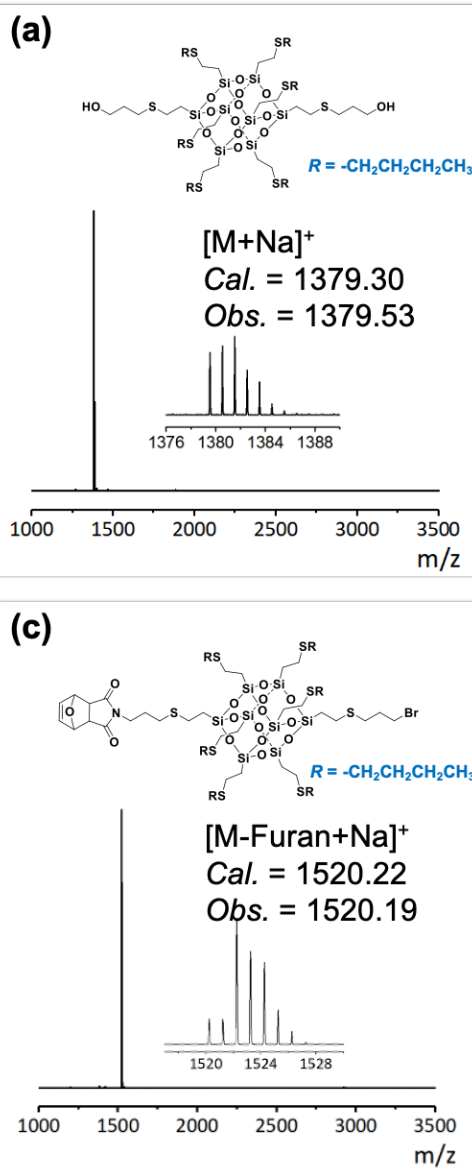

(b)

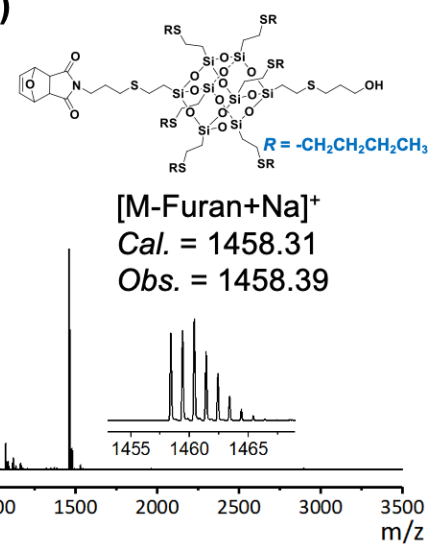

(d)

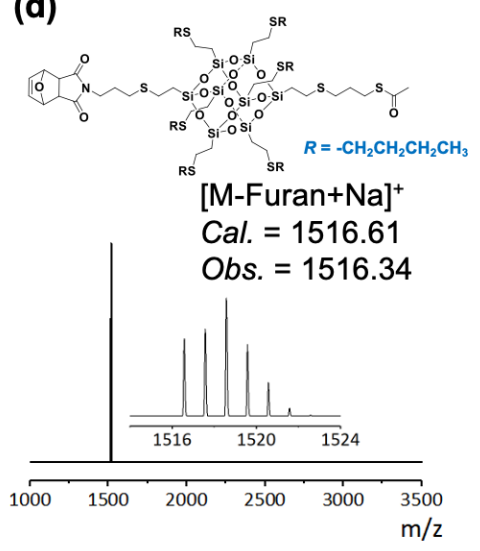

Figure S5. MALDI-ToF MS of the intermediates of $p$-CPOSS monomer.

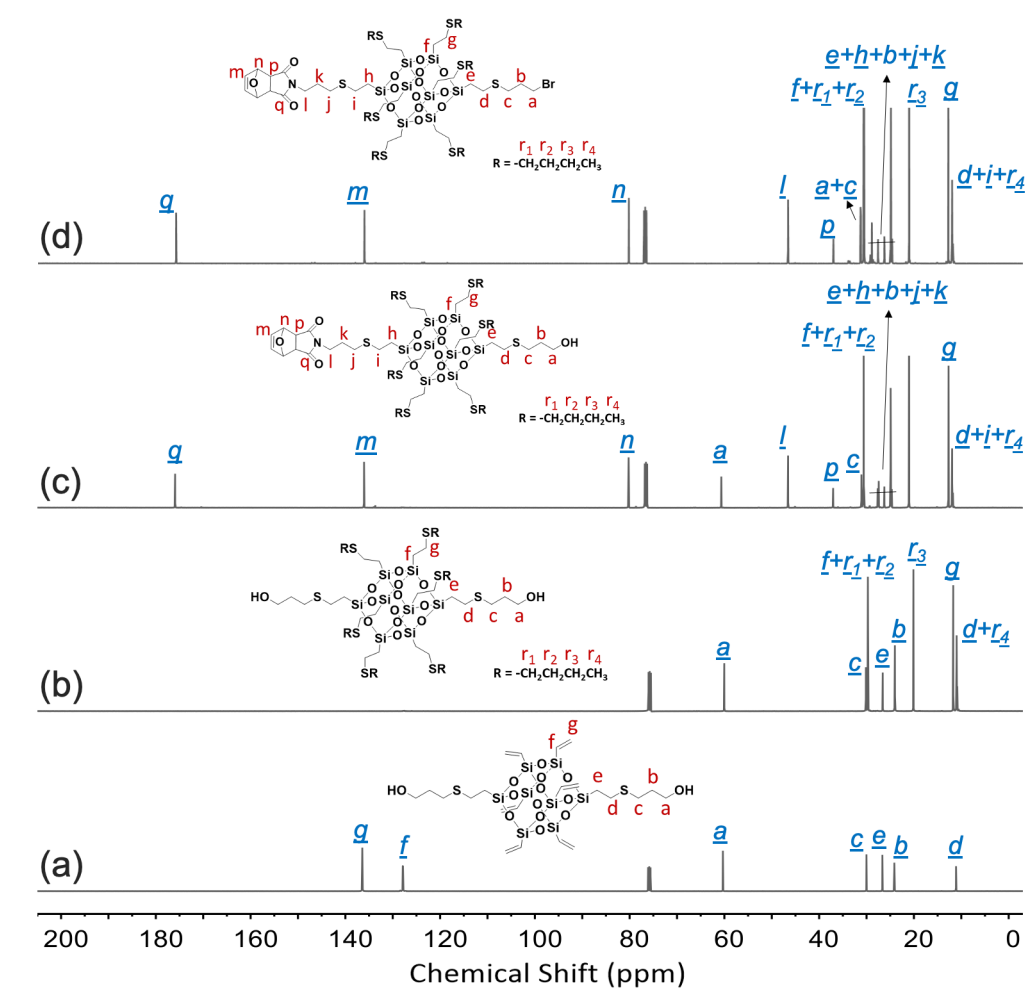

Figure S6. ${ }^{13} \mathrm{C}$ NMR spectra of the intermediates of $p$-CPOSS monomer. 


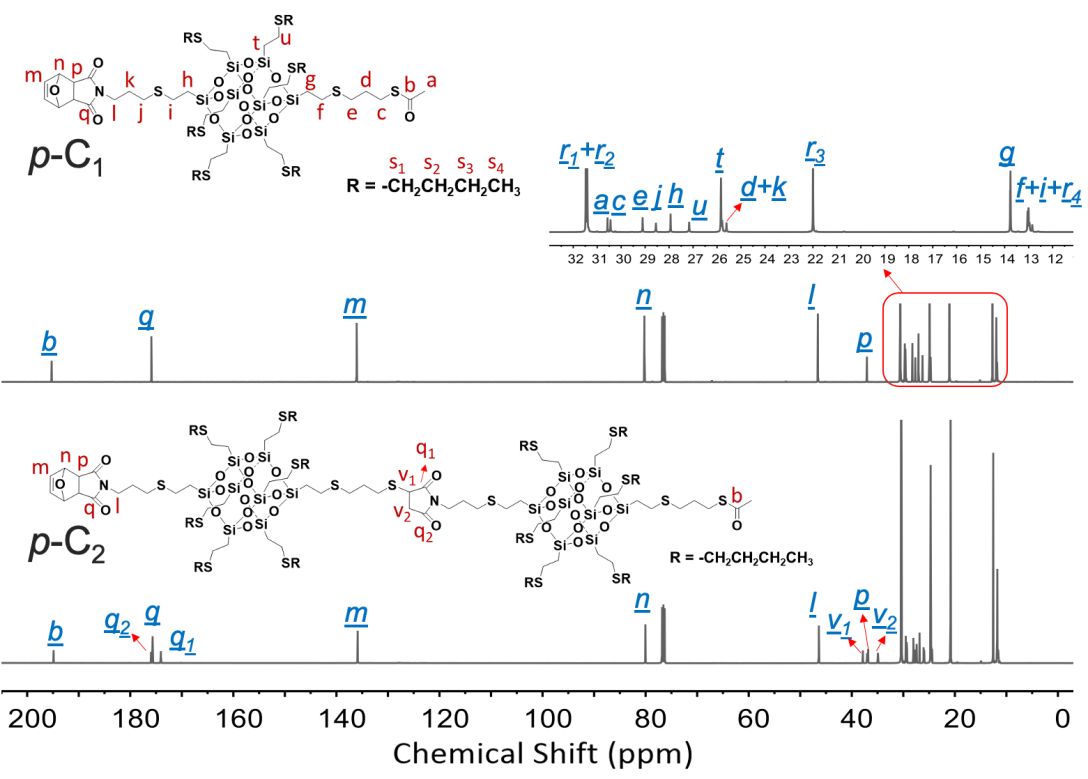

Figure S7. ${ }^{13} \mathrm{C}$ NMR spectra of the $p-\mathrm{C}_{1}$ and $p-\mathrm{C}_{2}$.

(a)
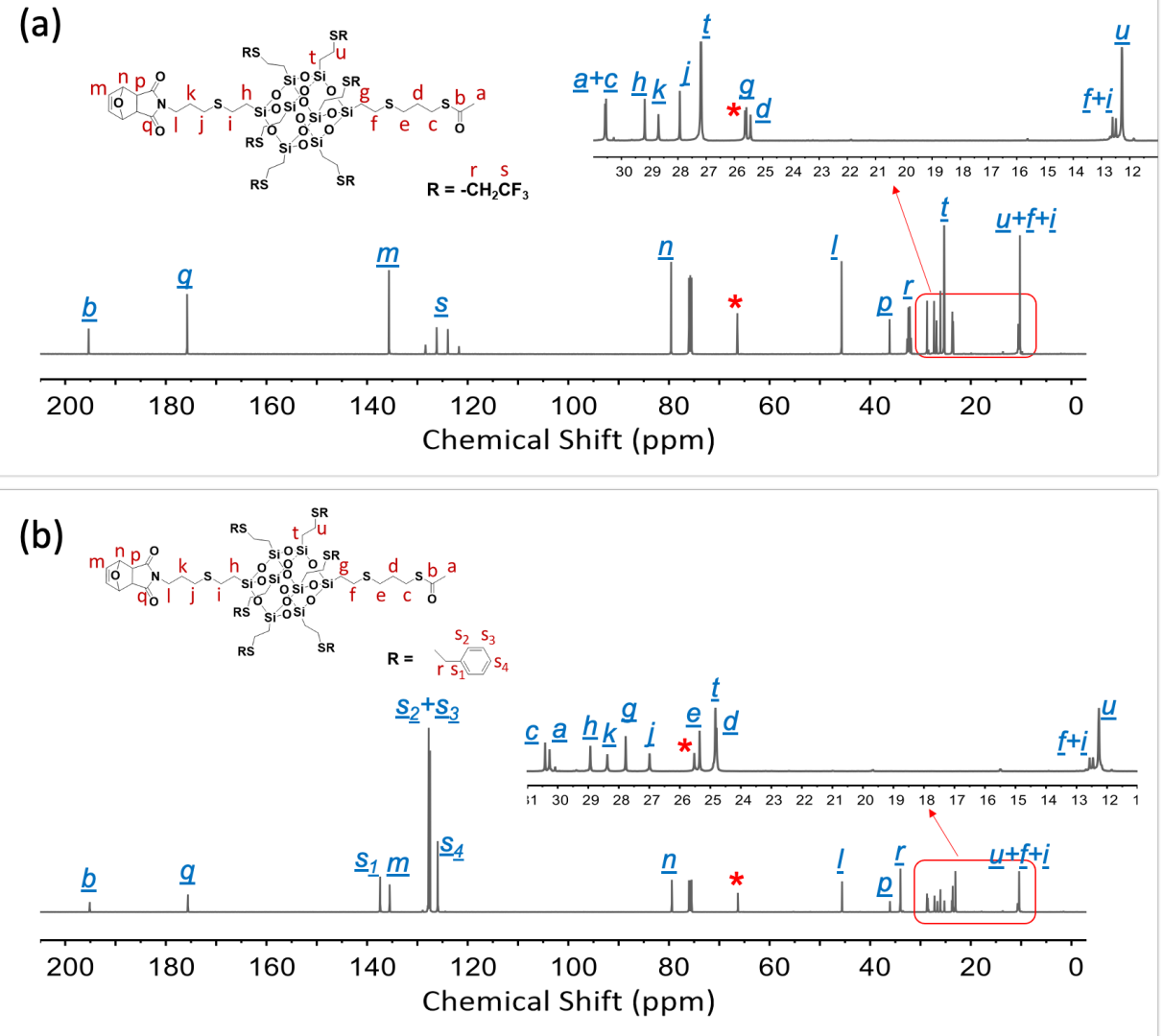

Figure S8. ${ }^{13} \mathrm{C}$ NMR spectra of the $p$-FPOSS-monomer (a) and $p$-PPOSS-monomer (b). The red asterisks denotes resonances from residual THF. 


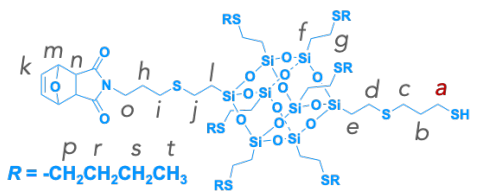

(c) $\underline{k}$ Im 으
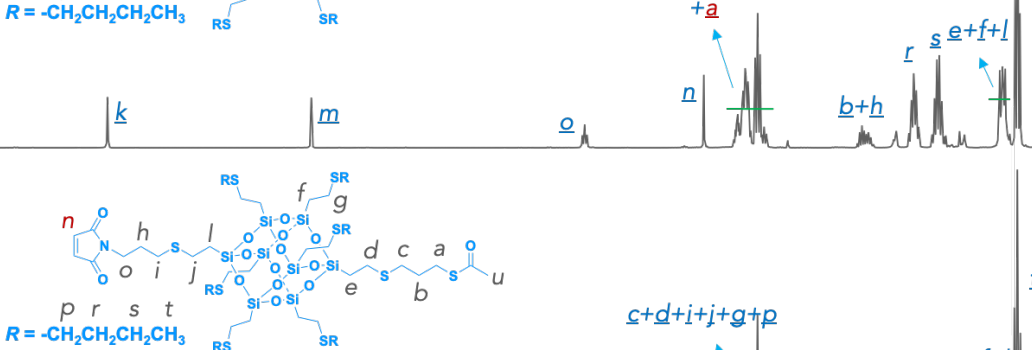

(b)
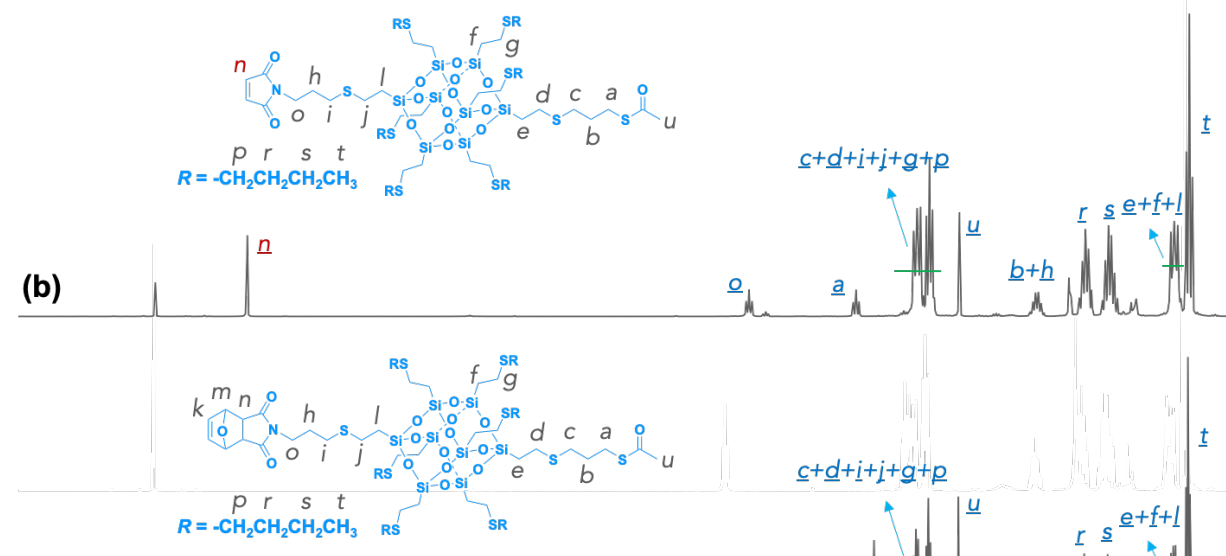

(a) $\underline{\underline{m}}$

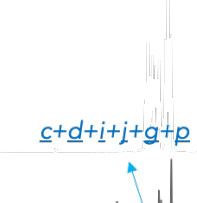
almanl

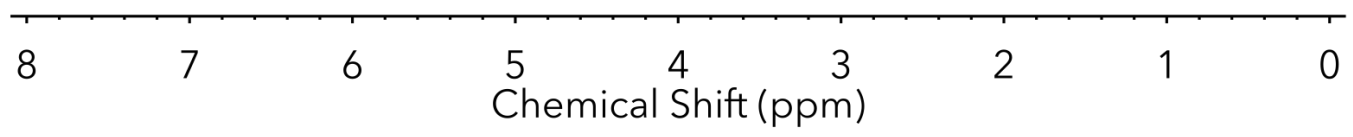

Figure S9. ${ }^{1} \mathrm{H}$ NMR of the orthogonal deprotection process: $p$ - $\mathrm{C}_{1}(\mathrm{a}), p-\mathrm{Mal}-\mathrm{C}_{1}$ (b), and $p-\mathrm{C}_{1}-\mathrm{SH}$ (c).

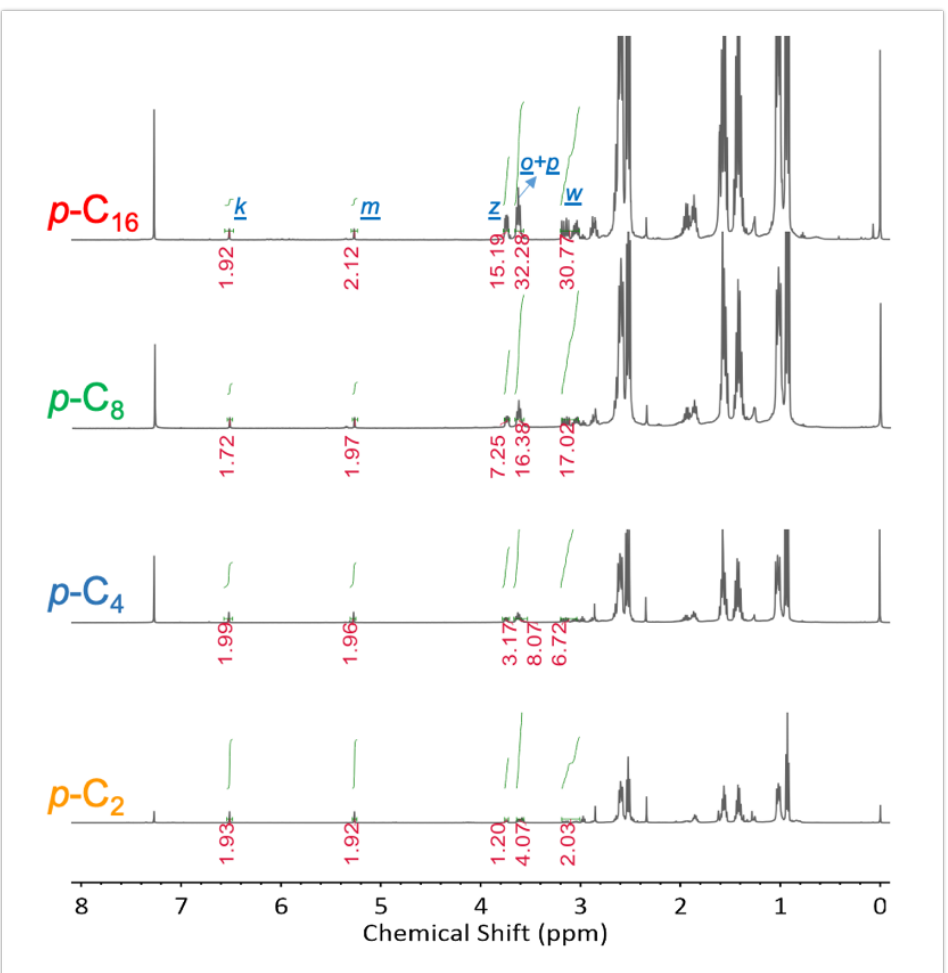

Figure S10. ${ }^{1} \mathrm{H}$ NMR spectra of discrete CPOSS chains with para-configuration $\left(p-\mathrm{C}_{n}\right)$. 


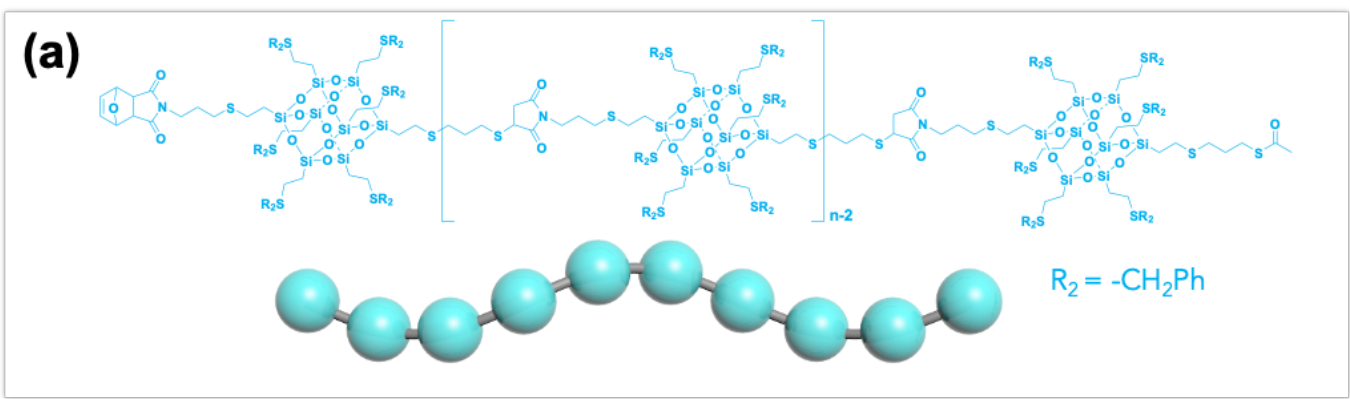

(b)
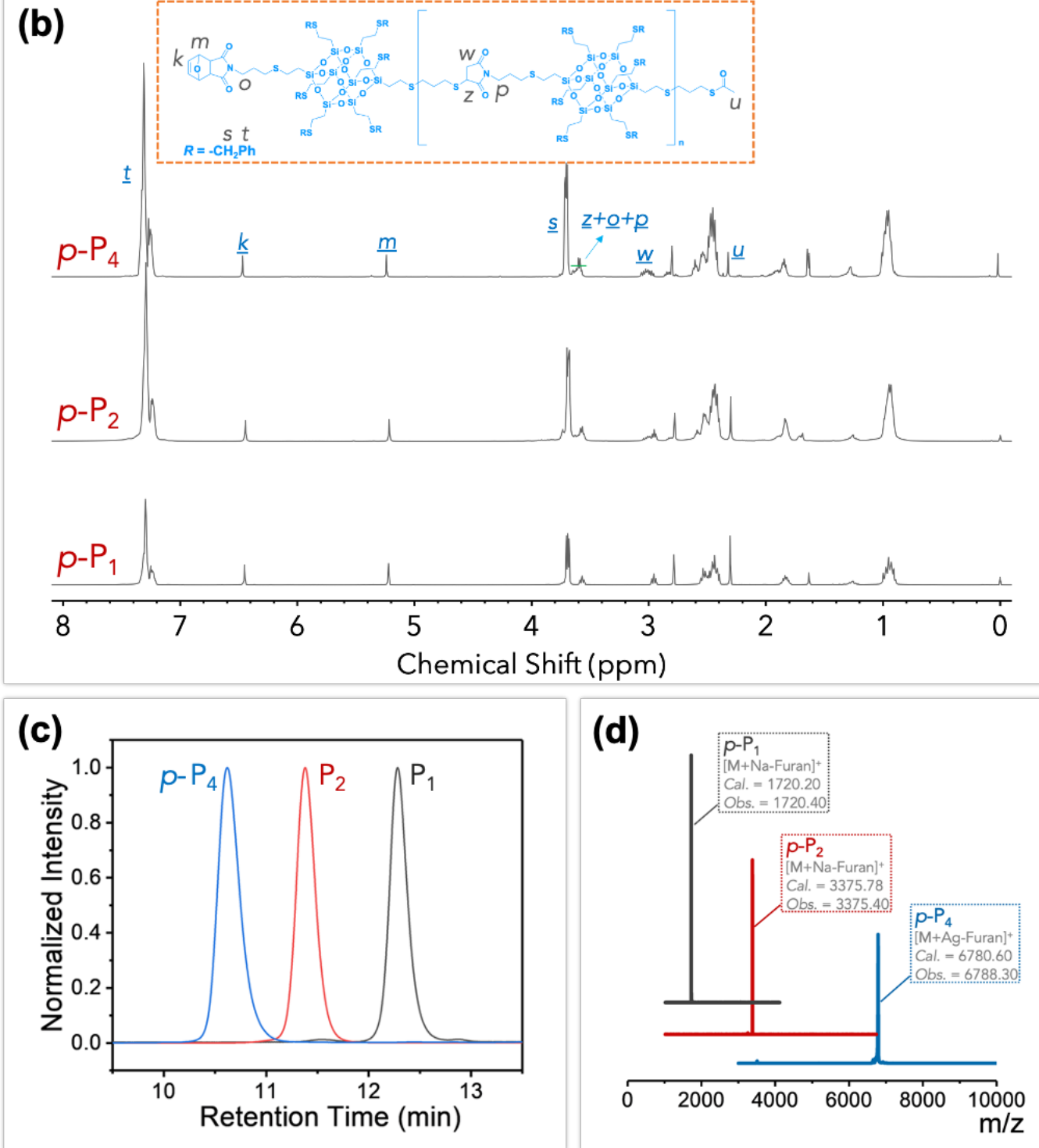

(d)

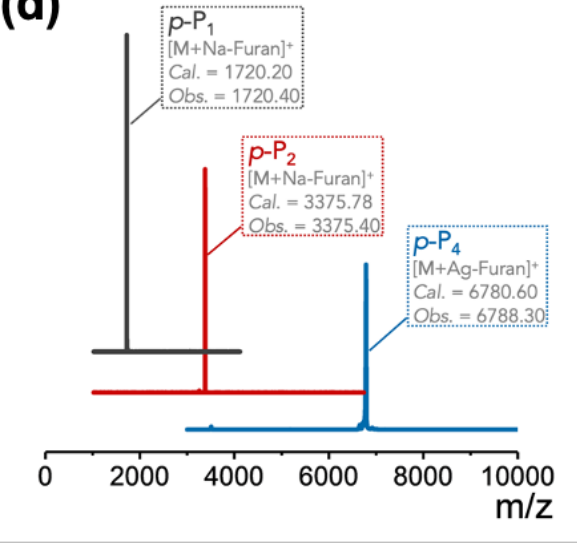

Figure S11. Chemical structure (a), ${ }^{1} \mathrm{H}$ NMR spectra (b), SEC profiles (c), and MALDI-ToF MS (d) of PPOSS chains $\left(p-\mathrm{P}_{n}\right)$. 
(a)

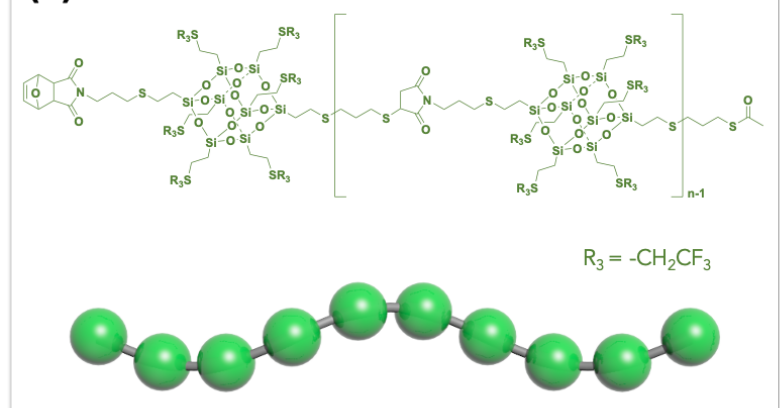

(b)

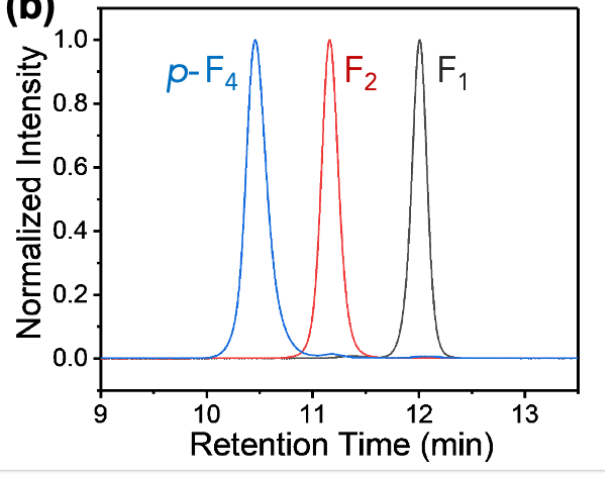

(c)
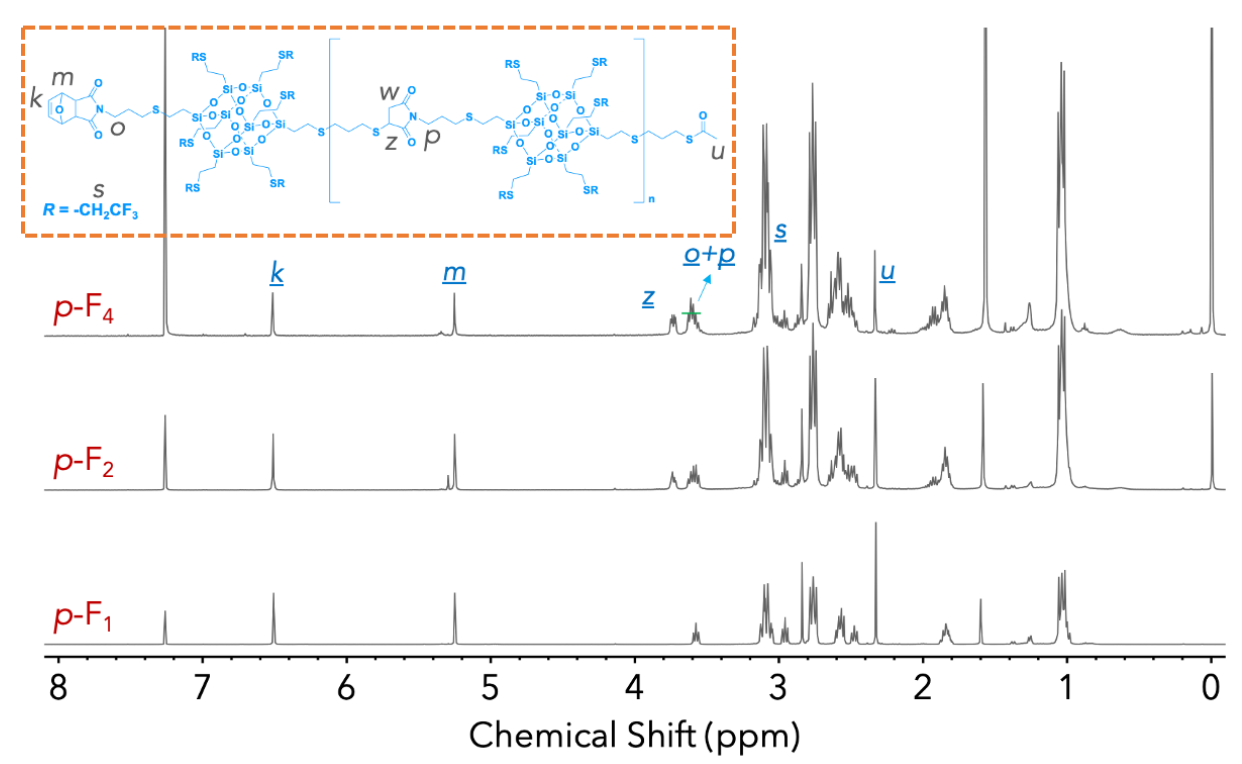

Figure S12. Chemical structure (a), SEC profiles (b), and ${ }^{1} \mathrm{H}$ NMR spectra (c) of FPOSS chains $\left(p-\mathrm{F}_{n}\right)$. 

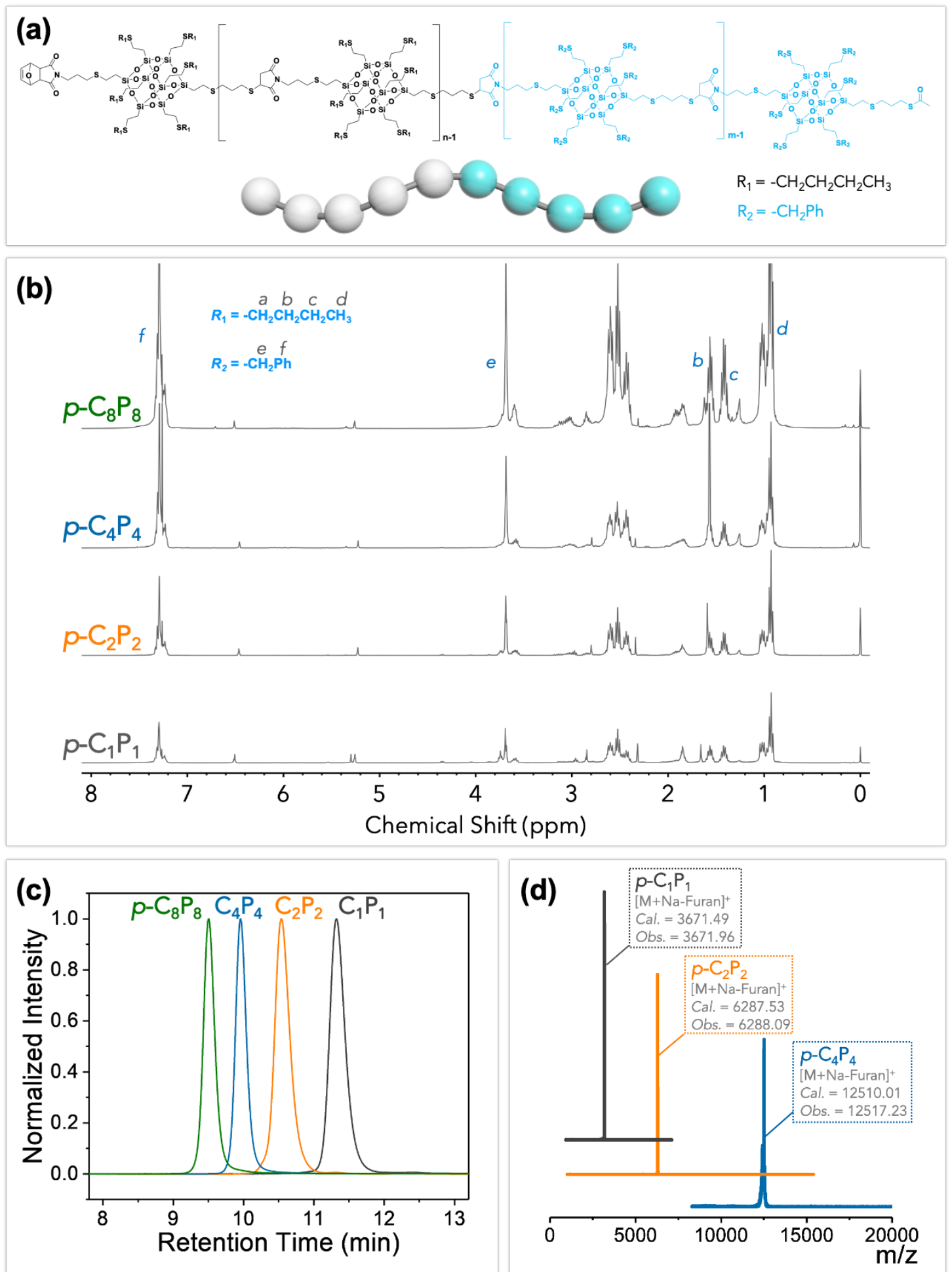

Figure S13. Chemical Structure (a), ${ }^{1} \mathrm{H}$ NMR spectra (b), SEC profiles (c), and MALDI-ToF MS (d) of $p$ - ${ }_{n} \mathrm{P}_{n}$ chains. 

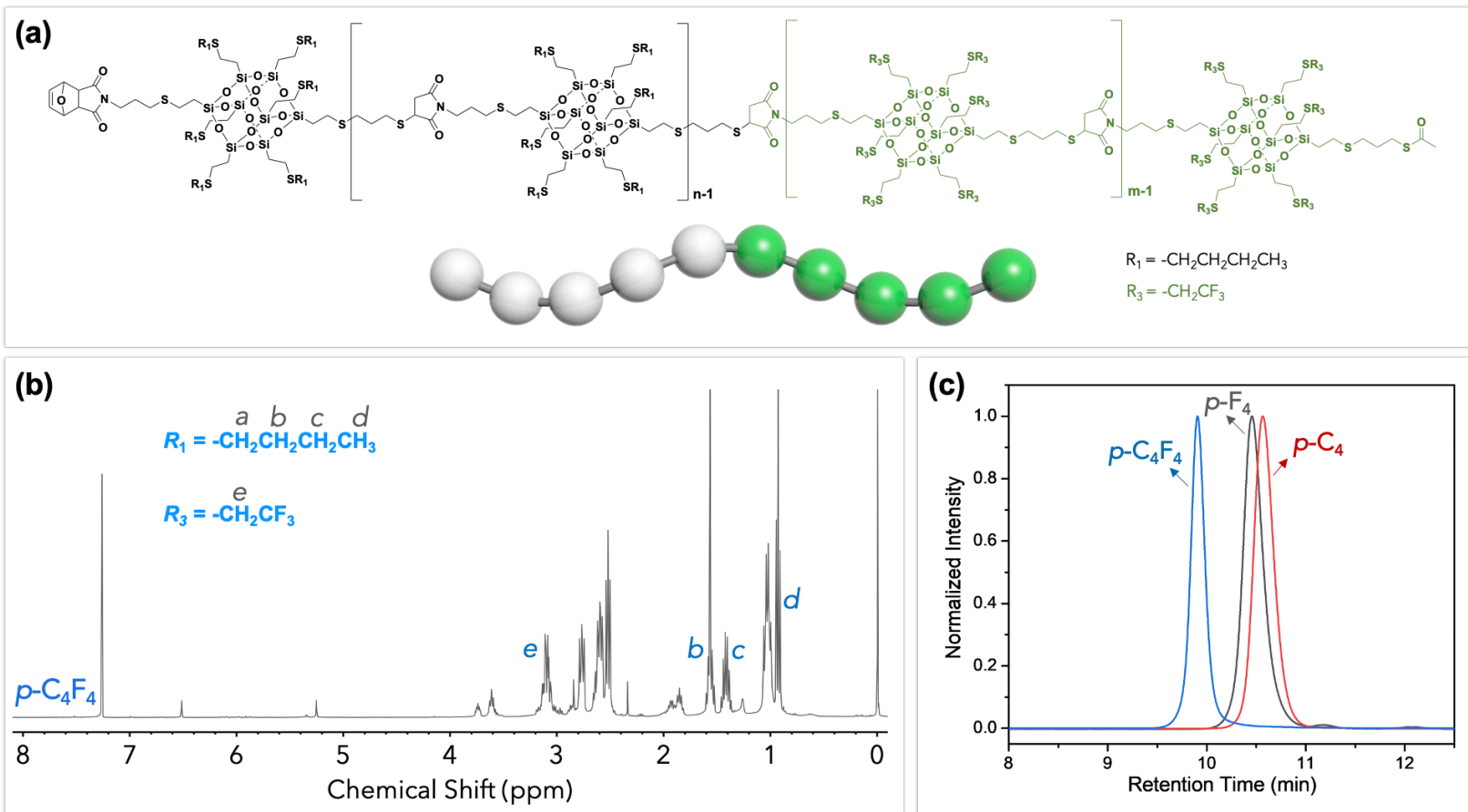

Figure S14. Chemical Structure (a), ${ }^{1} \mathrm{H}$ NMR spectra (b), and SEC profiles (c) of $p-\mathrm{C}_{n} \mathrm{~F}_{n}$ chains. 

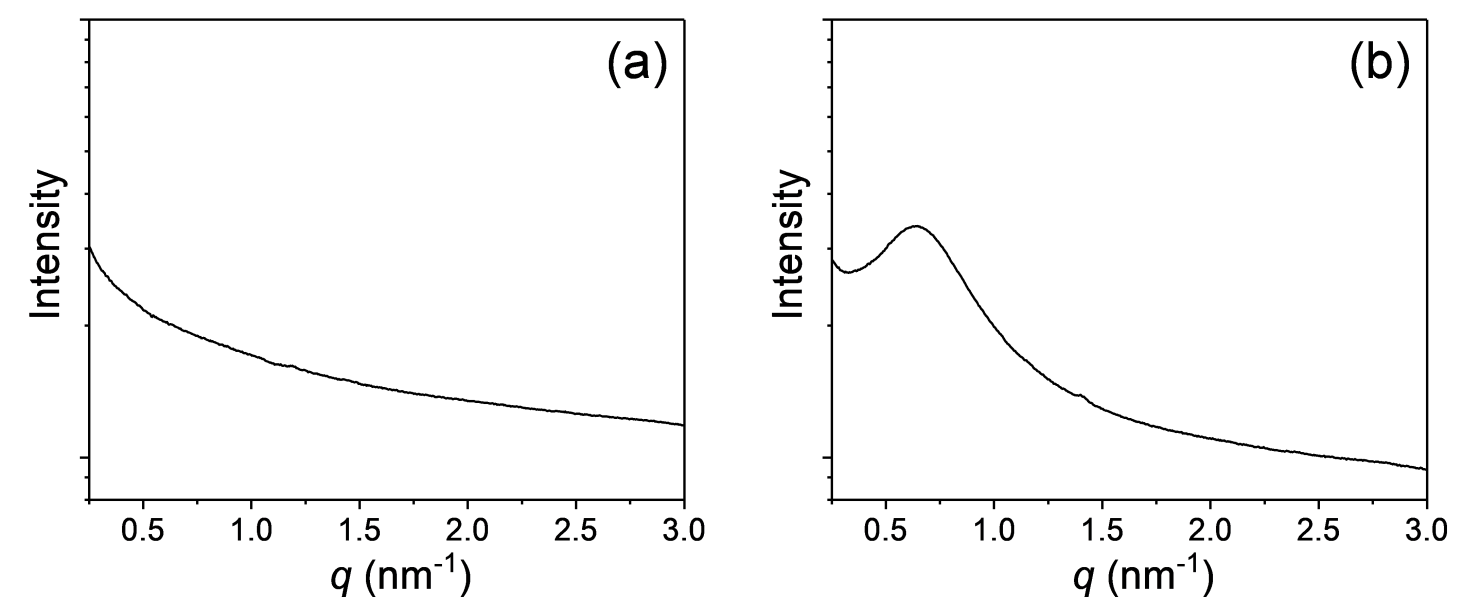

Figure S15. SAXS profiles of $p-\mathrm{C}_{2} \mathrm{P}_{2}(\mathrm{a})$ and $p-\mathrm{C}_{4} \mathrm{~F}_{4}$ (b) at room temperature.

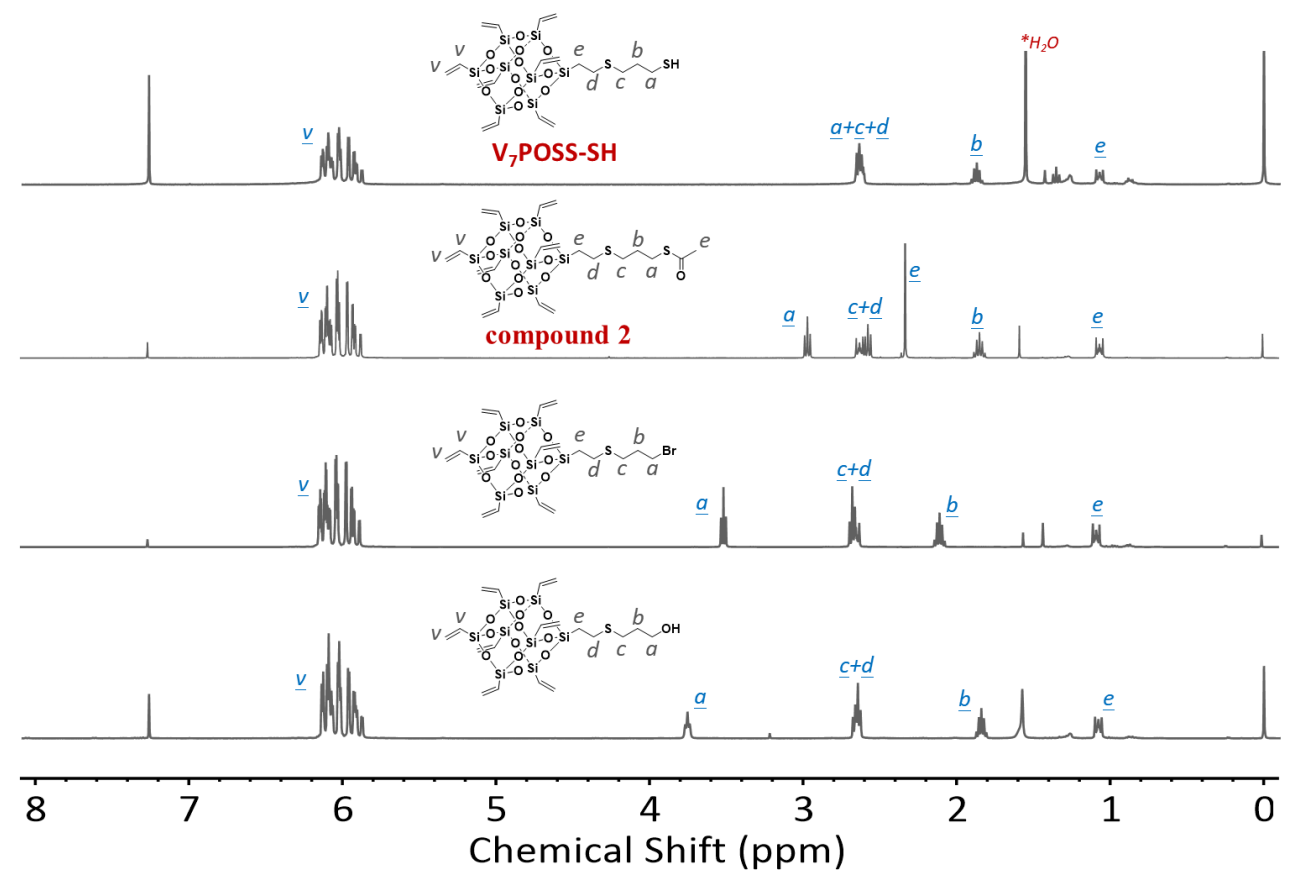

Figure S16. ${ }^{1} \mathrm{H}$ NMR spectra of $\mathrm{V}_{7} \mathrm{POSS}-\mathrm{SH}$ and its precursors. 

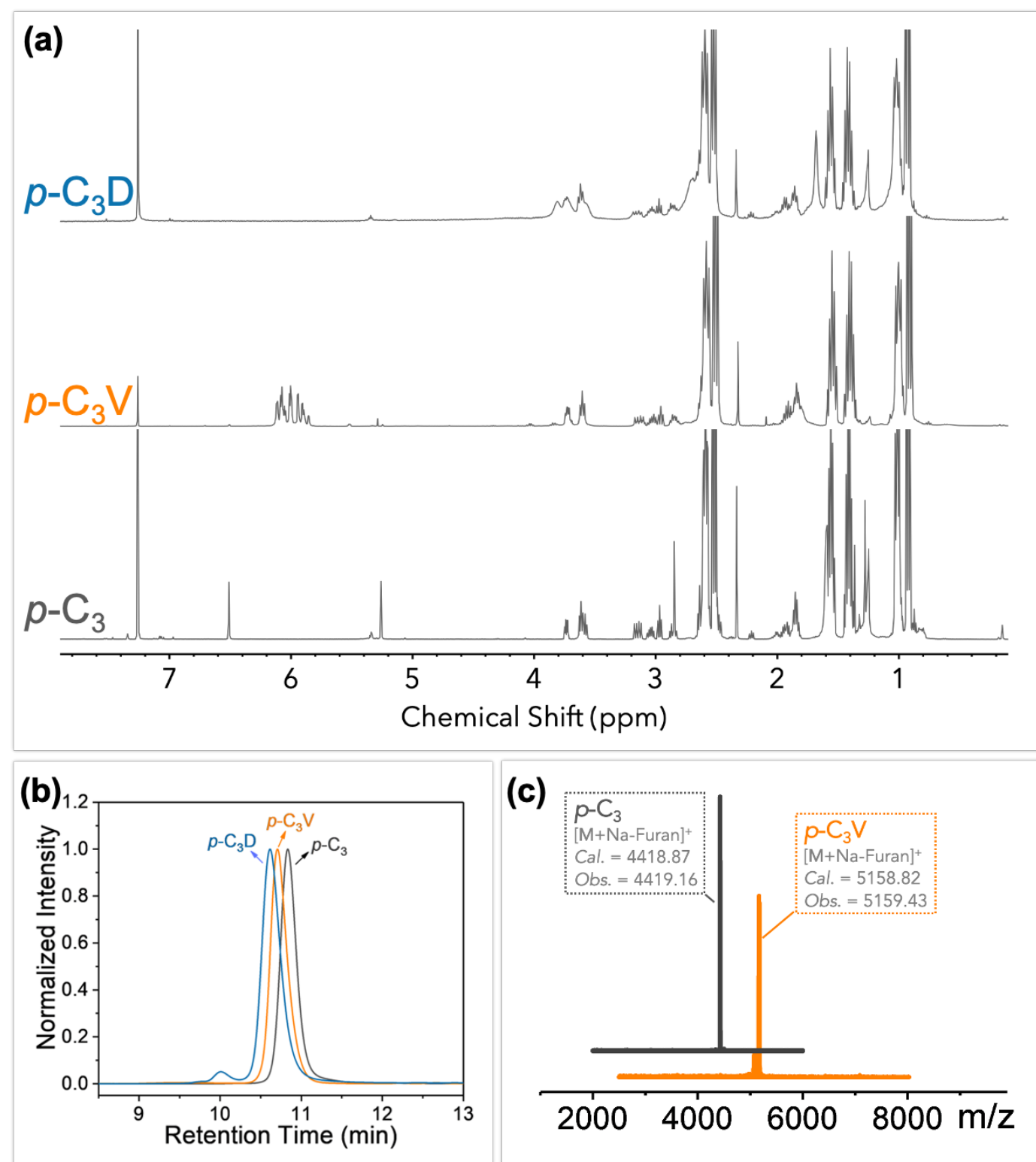

Figure S17. ${ }^{1} \mathrm{H}$ NMR spectra (a), SEC profiles (b), and MALDI-ToF MS (c) of $p$ - ${ }_{n} \mathrm{D}$ (take $n=3$ as an example). 


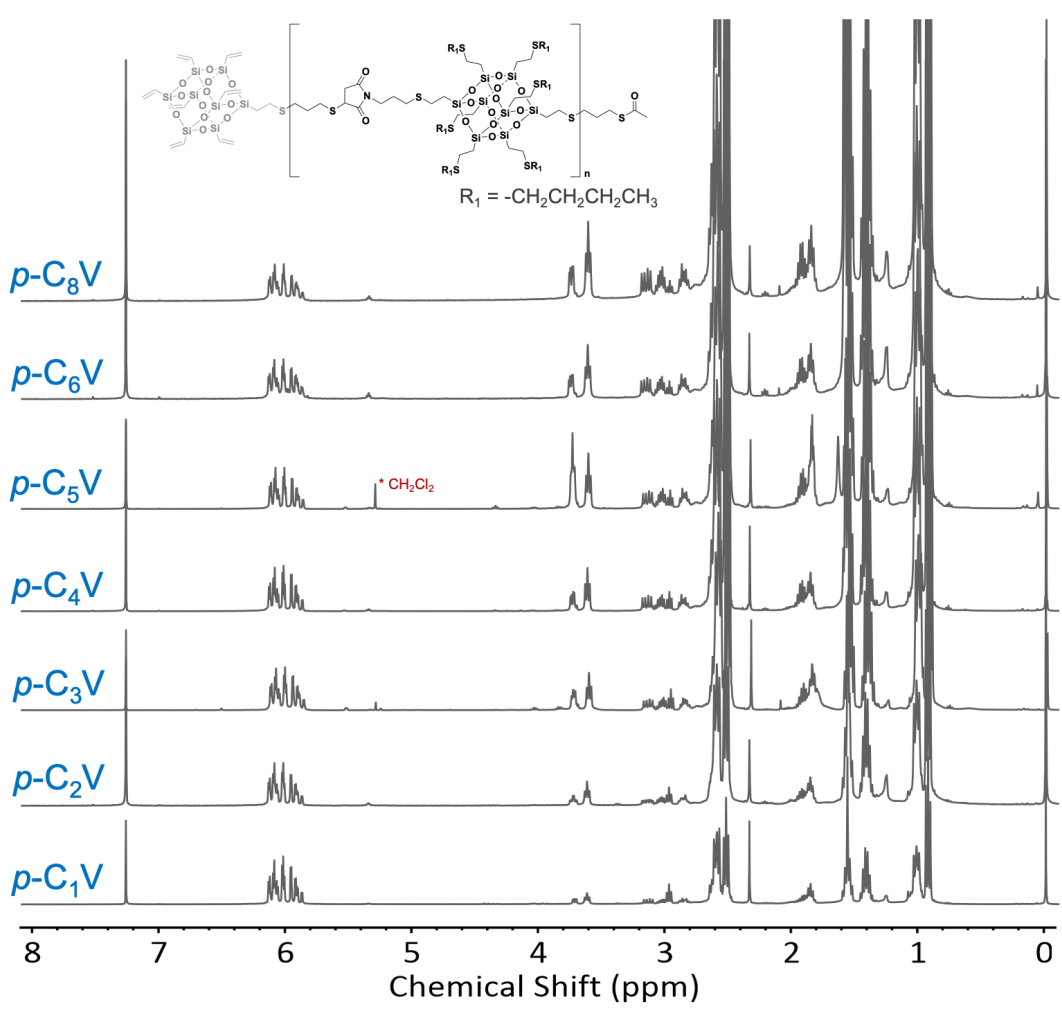

Figure S18. ${ }^{1} \mathrm{H}$ NMR spectra of $p-\mathrm{C}_{n} \mathrm{~V}$ with varied number of repeat units.

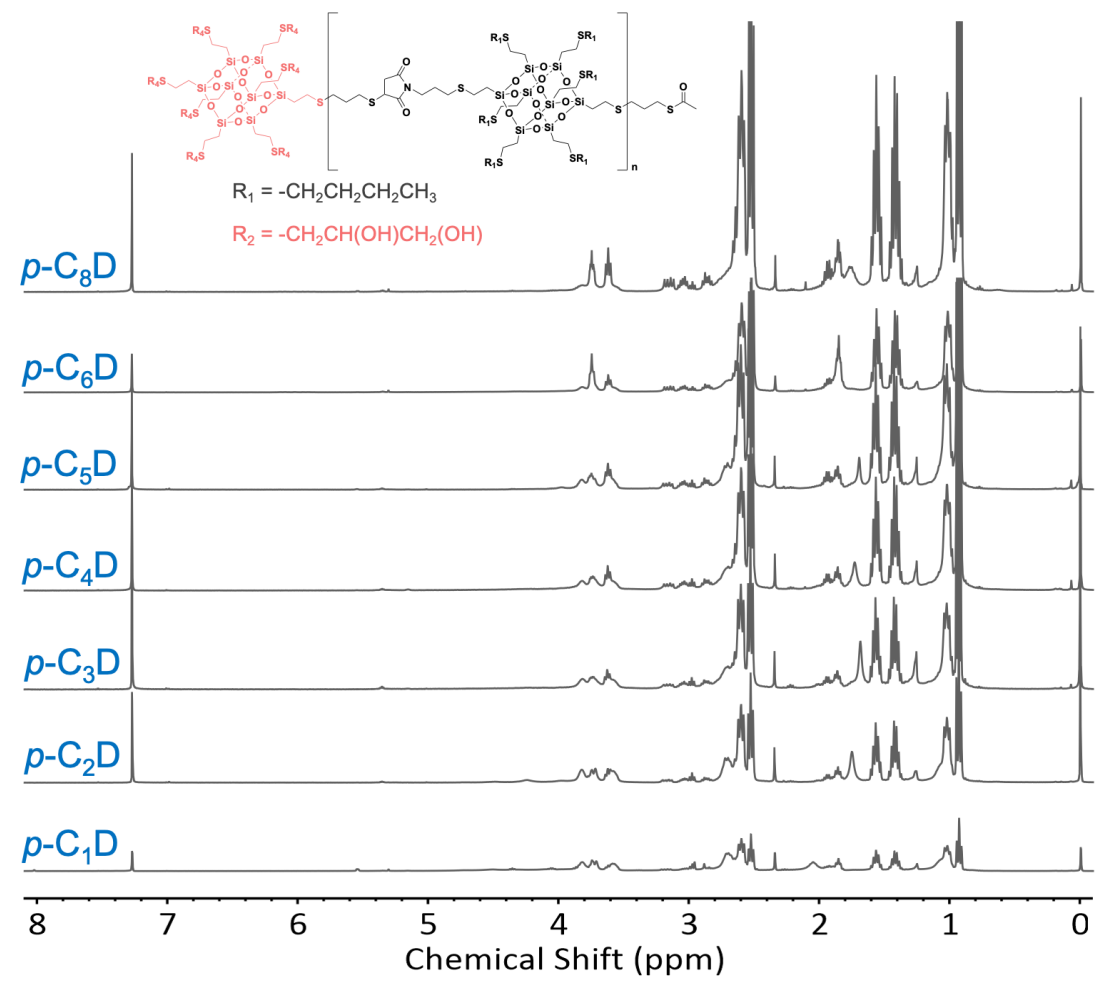

Figure S19. ${ }^{1} \mathrm{H}$ NMR spectra of $p-\mathrm{C}_{n} \mathrm{D}$ with varied number of repeat units. 

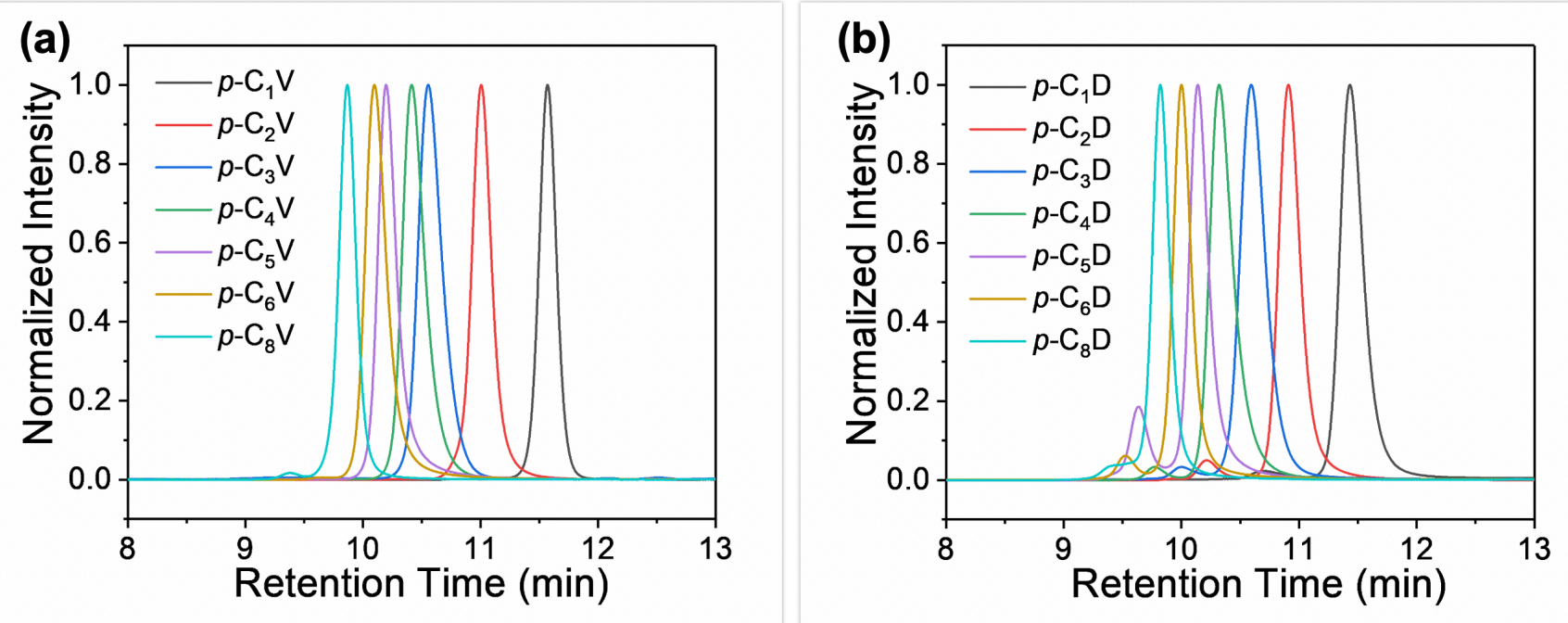

Figure S20. SEC profiles of $p-\mathrm{C}_{n} \mathrm{~V}(\mathrm{a})$ and $p-\mathrm{C}_{n} \mathrm{D}(\mathrm{b})$.
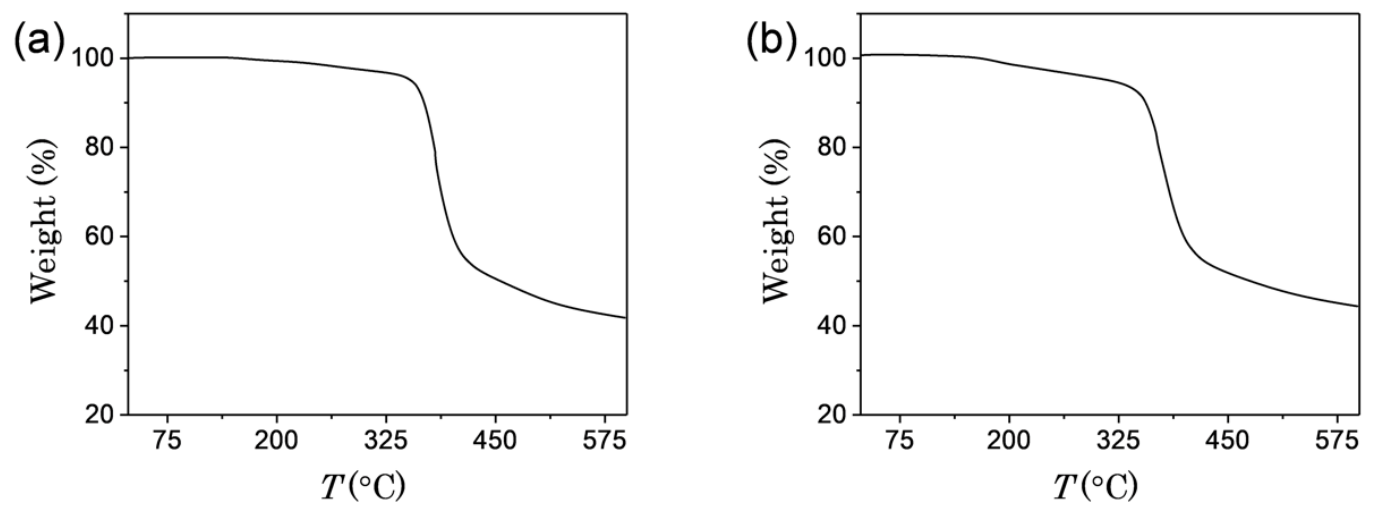

Figure S21. TGA curves of $p-\mathrm{C}_{4}(\mathrm{a})$ and $p-\mathrm{C}_{8} \mathrm{D}(\mathrm{b})$. Heating rate: $10^{\circ} \mathrm{C} / \mathrm{min}$. 

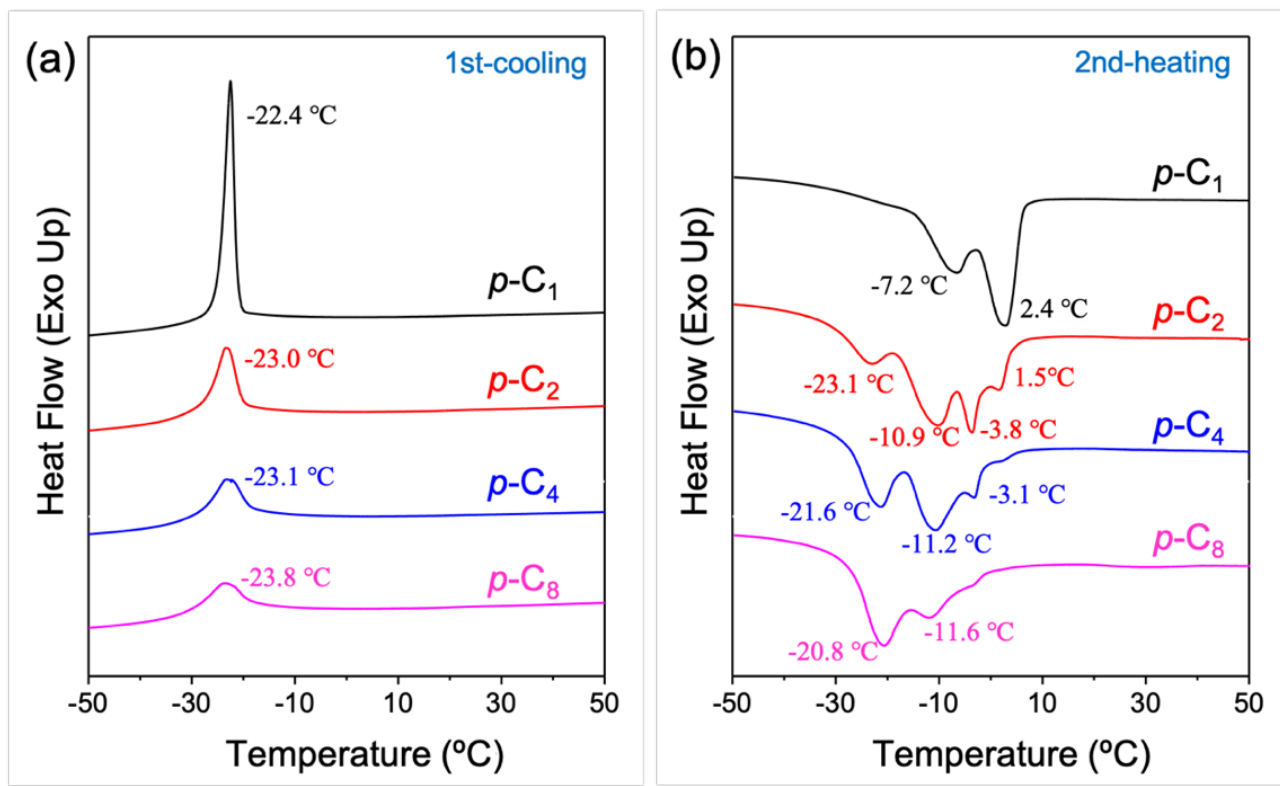

Figure S22. DSC thermograms of the $p-\mathrm{C}_{n}$ in the cooling (a) and heating (b) cycle. Heating/cooling rate: $5{ }^{\circ} \mathrm{C} / \mathrm{min}$.
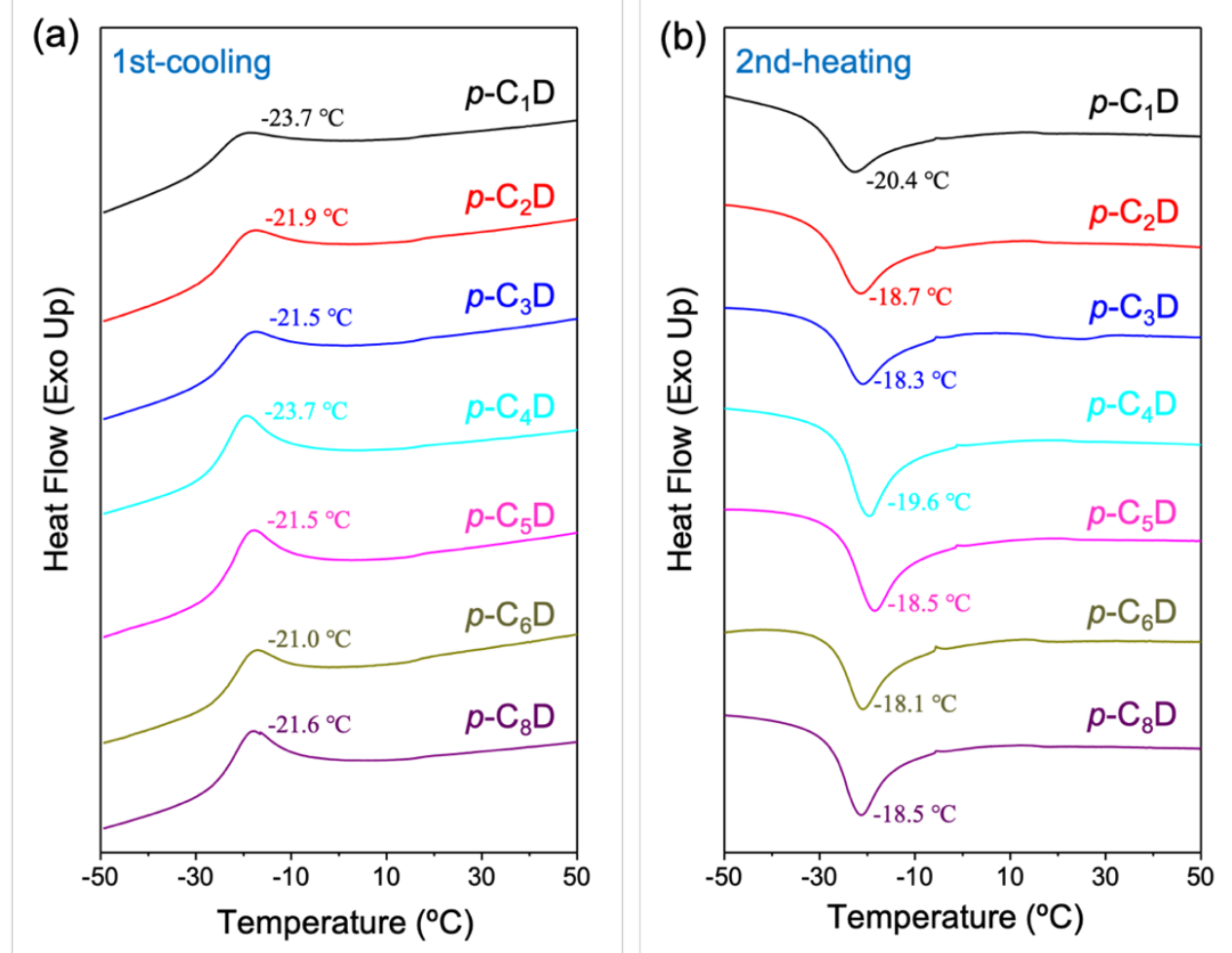

Figure S23. DSC thermograms of the $p-\mathrm{C}_{n} \mathrm{D}$ in the cooling (a) and heating (b) cycle. Heating/cooling rate: $5^{\circ} \mathrm{C} / \mathrm{min}$. 

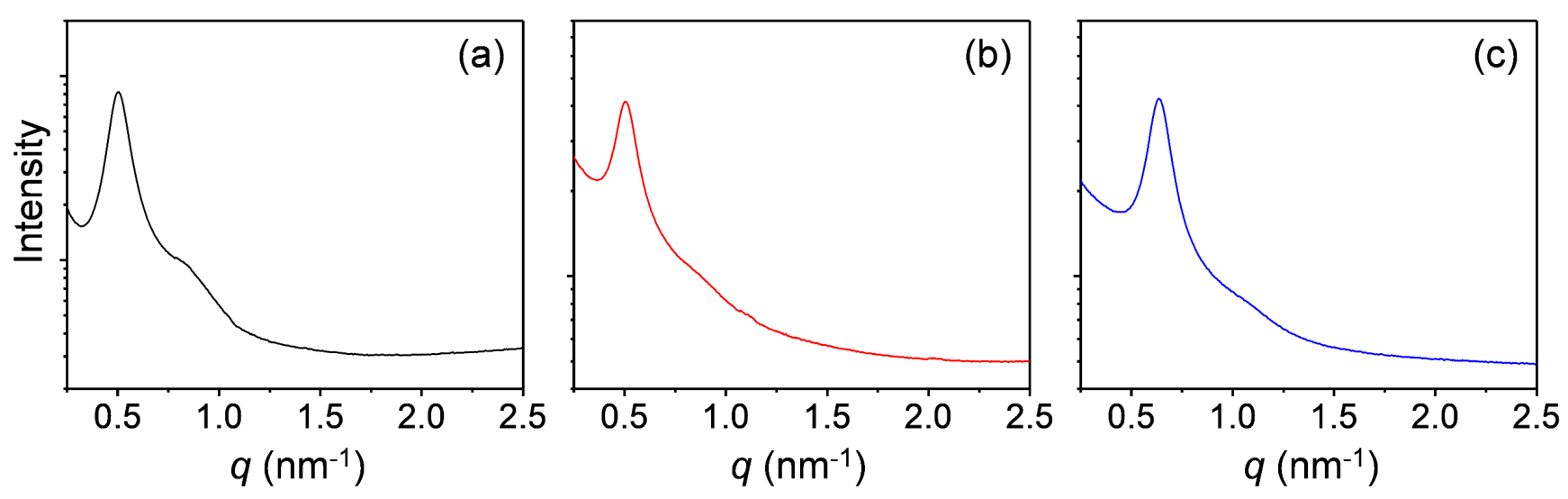

Figure S24. SAXS patterns of $p-\mathrm{C}_{8} \mathrm{D}(\mathrm{a}), m-\mathrm{C}_{8} \mathrm{D}(\mathrm{b})$, and $o-\mathrm{C}_{8} \mathrm{D}(\mathrm{c})$.
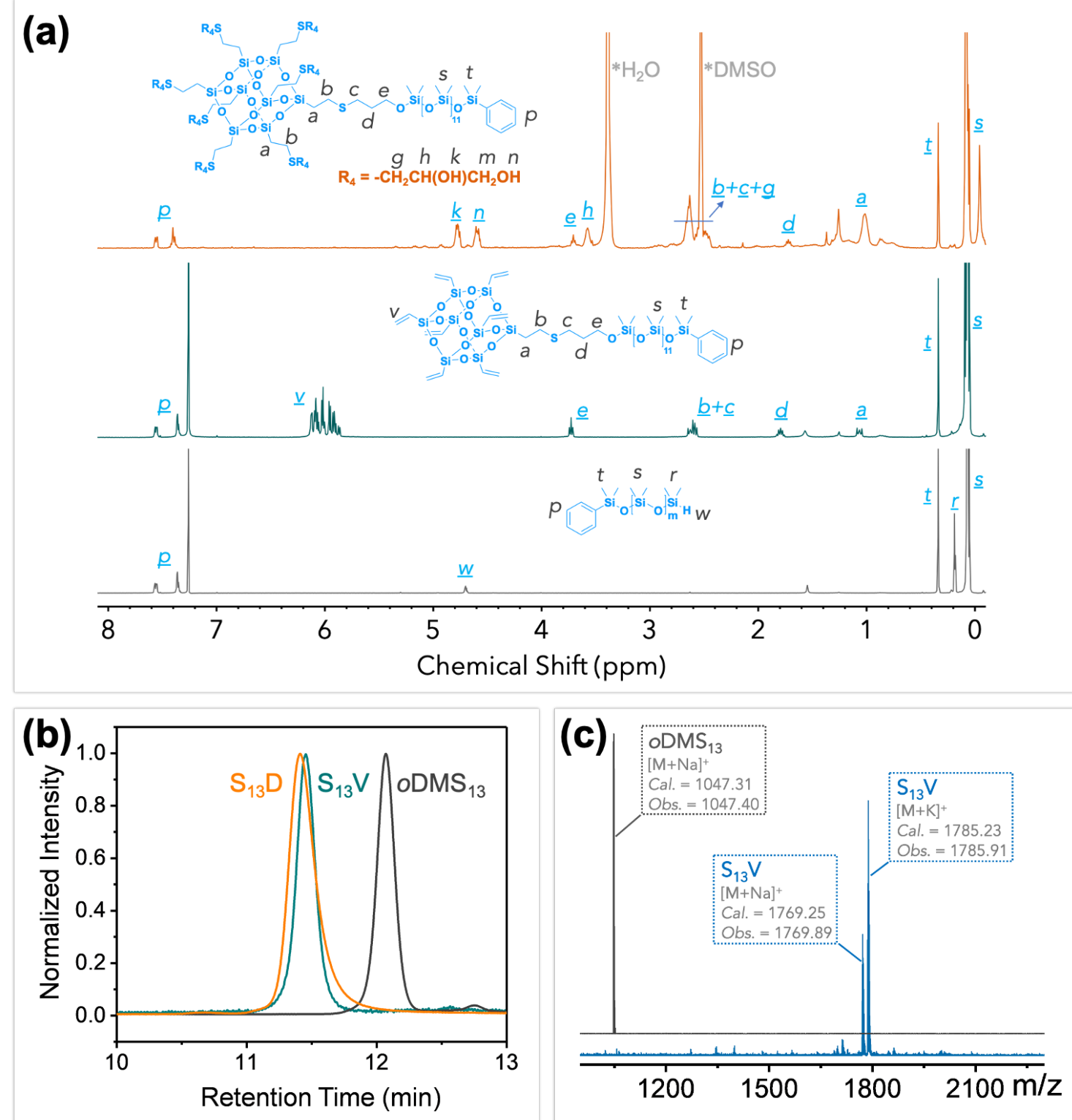

Figure S25. ${ }^{1} \mathrm{H}$ NMR spectra (a), SEC profiles (b), and MALDI-ToF MS (c) of $\mathrm{S}_{13} \mathrm{D}$ and its precursors. 

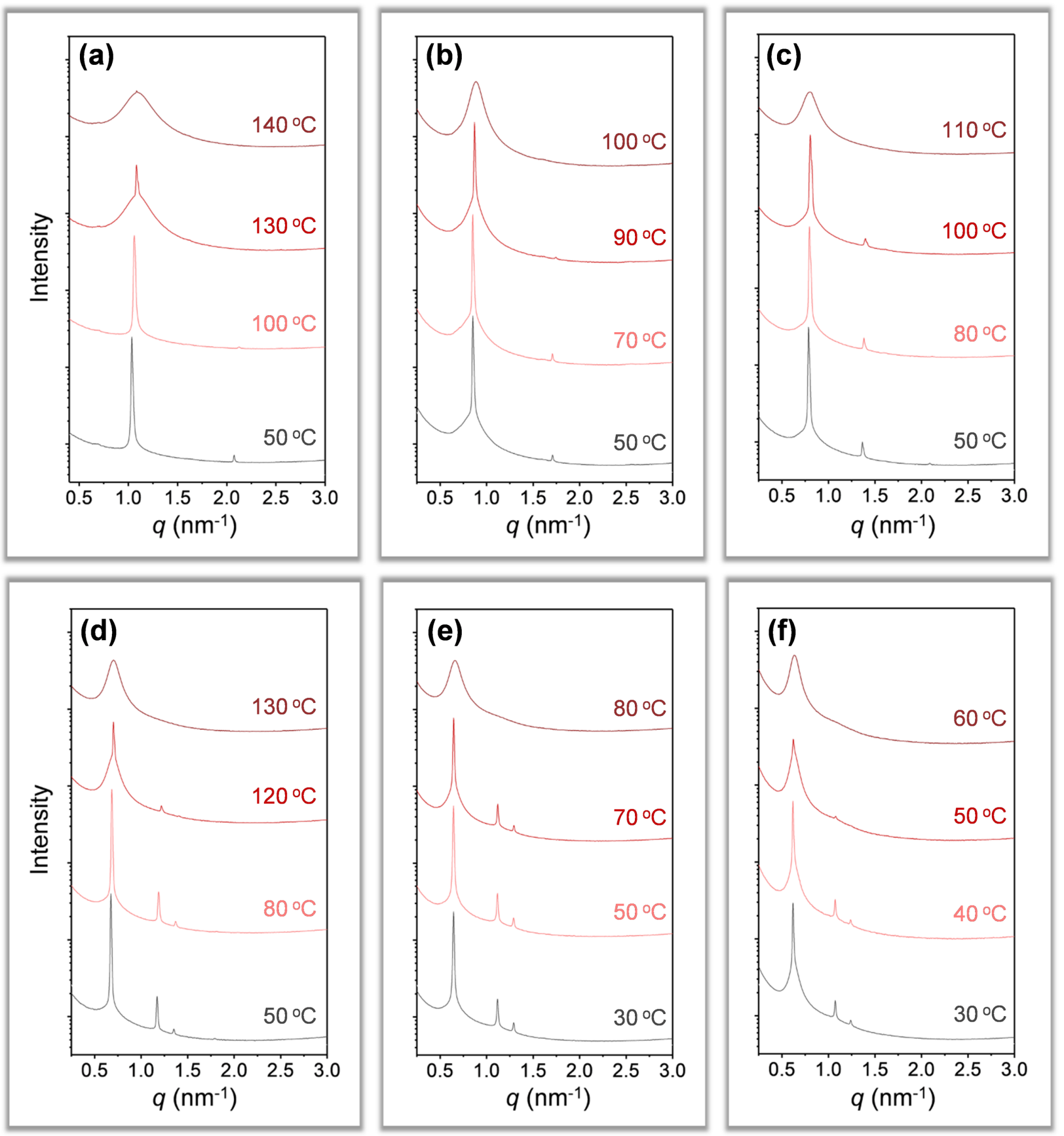

Figure S26. Temperature dependent SAXS profiles of the amphiphilic POSS chains: $p-\mathrm{C}_{1} \mathrm{D}(\mathrm{a}), p-\mathrm{C}_{2} \mathrm{D}(\mathrm{b}), p-\mathrm{C}_{3} \mathrm{D}(\mathrm{c})$, $p-\mathrm{C}_{4} \mathrm{D}(\mathrm{d}), p-\mathrm{C}_{5} \mathrm{D}(\mathrm{e})$, and $p-\mathrm{C}_{6} \mathrm{D}(\mathrm{f})$. 


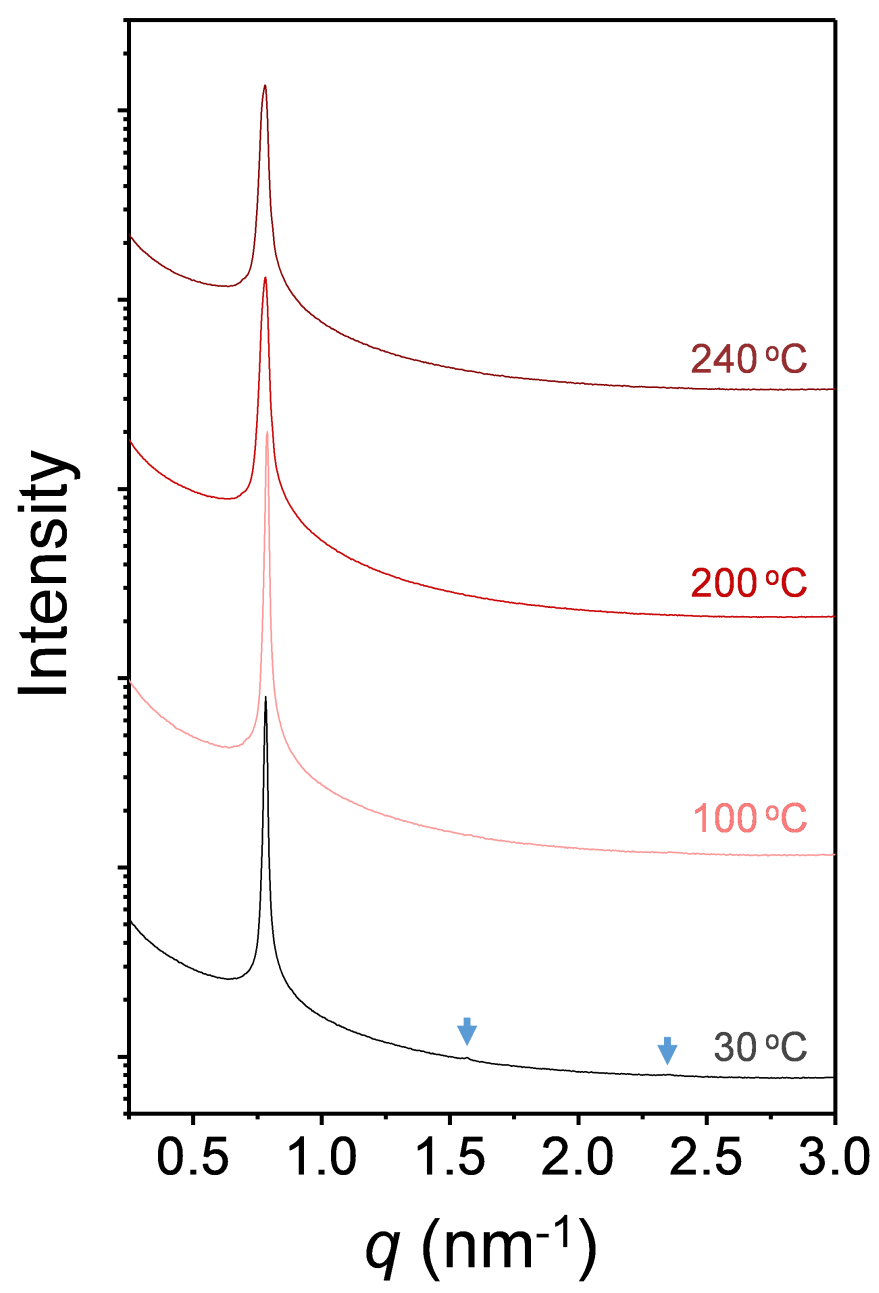

Figure S27. Temperature dependent SAXS profiles of the polymer-particle conjugate $\mathrm{S}_{13} \mathrm{D}$. 


\section{References}

[1] Feng, X.; Zhang R.; Li Y.; Hong, Y-L.; Guo, D.; Lang, K.; Wu, K.-Y.; Huang, M.; Mao, J.; Wesdemiotis, C.; Nishiyama, Y.;

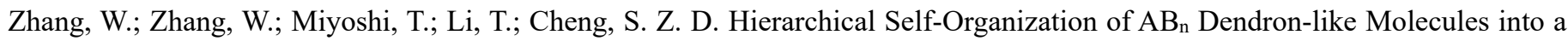
Supramolecular Lattice Sequence. ACS Cent Sci, 2017, 3, 860-867.

[2] van Genabeek, B.; de Waal, B. F. M.; Gosens, M. M. J.; Pitet, L. M.; Palmans, A. R. A.; Meijer, E. W. Synthesis and SelfAssembly of Discrete Dimethylsiloxane-Lactic Acid Diblock Co-Oligomers: The Dononacontamer and Its Shorter Homologues. $J$ Am Chem Soc 2016, 138 (12), 4210-4218.

[3] Zhang, W.; Zhang, S. L.; Guo, Q. Y.; Lu, X. L.; Liu, Y. C.; Mao, J. L.; Wesdemiotis, C.; Li. T.; Li, Y. W.; Cheng, S. Z. D. Multilevel Manipulation of Supramolecular Structures of Giant Molecules via Macromolecular Composition and Sequence. ACS Macro Lett. 2018, 7, 635-640.

[4] Zhang, W.; Huang, M. J.; Su, H.; Zhang, S.; Yue, K.; Dong, X. H.; Li, X.; Liu, H.; Zhang, S.; Wesdemiotis, C.; Lotz, B.; Zhang, W. B.; Li, Y.; Cheng, S. Z. D. Toward Controlled Hierarchical Heterogeneities in Giant Molecules with Precisely Arranged Nano Building Blocks. ACS Cent. Sci. 2016, 2, 48-54.

[5] Zhang, W.; Shan, W.; Zhang, S.; Liu, Y.; Su, H.; Luo, J.; Xia, Y.; Li, T.; Wesdemiotis, C.; Liu, T.; Cui, H.; Li, Y.; Cheng, S. Z. D. Sequence isomeric giant surfactants with distinct self-assembly behaviors in solution. Chem. Commun. 2019, 55, 636-639. 